\title{
Cosmicflows-4: The Calibration of Optical and Infrared Tully-Fisher Relations
}

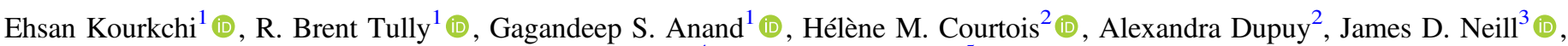 \\ Luca Rizzi ${ }^{4}$ (D), and Mark Seibert ${ }^{5}$ (i) \\ ${ }^{1}$ Institute for Astronomy, University of Hawaii, 2680 Woodlawn Drive, Honolulu, HI 96822, USA; ehsan@ifa.hawaii.edu, tully@ifa.hawaii.edu \\ ${ }^{2}$ University of Lyon, UCB Lyon 1, CNRS/IN2P3, IUF, IP2I Lyon, France \\ ${ }^{3}$ California Institute of Technology, 1200 East California Boulevard, MC 278-17, Pasadena, CA 91125, USA \\ ${ }^{4}$ W.M. Keck Observatory, 65-1120 Mamalahoa Highway, Kamuela, HI 96743, USA \\ ${ }^{5}$ The Observatories of the Carnegie Institute of Washington, 813 Santa Barbara Street, Pasadena, CA 91101, USA \\ Received 2020 February 4; revised 2020 April 9; accepted 2020 April 29; published 2020 June 8
}

\begin{abstract}
This study is a part of the Cosmicflows-4 project with the aim of measuring the distances of more than $\sim 10,000$ spiral galaxies in the local universe up to $\sim 15,000 \mathrm{~km} \mathrm{~s}^{-1}$. New H I line width information has come primarily from the Arecibo Legacy Fast ALFA Survey. Photometry of our sample galaxies has been carried out in optical (SDSS $u, g, r, i$, and $z$ ) and infrared (WISE $W 1$ and $W 2$ ) bands. Inclinations have been determined using an online graphical interface accessible to a collaboration of citizen scientists. Galaxy distances are measured based on the correlation between the rotation rate of spirals and their absolute luminosity, known as the Tully-Fisher relation (TFR). In this study, we present the calibration of the TFR using a subsample of $\sim 600$ spirals located in 20 galaxy clusters. Correlations among such observables as color, surface brightness, and relative $\mathrm{H}$ I content are explored in an attempt to reduce the scatter about the TFR with the goal of obtaining more accurate distances. A preliminary determination of the Hubble constant from the distances and velocities of the calibrator clusters is $H_{0}=76.0 \pm 1.1$ (stat.) \pm 2.3 (sys.) $\mathrm{km} \mathrm{s}^{-1} \mathrm{Mpc}^{-1}$.
\end{abstract}

Unified Astronomy Thesaurus concepts: Distance measure (395); Galaxy distances (590); Galaxy photometry (611); H I line emission (690); Spiral galaxies (1560); Inclination (780); Galaxy structure (622); Large-scale structure of the universe (902)

Supporting material: machine-readable tables

\section{Introduction}

Through the Cosmicflows program, the dark matter distribution in the universe is reconstructed based on the peculiar motions of galaxies in response to the underlying gravitational field. The peculiar velocities of galaxies are calculated by subtracting the Hubble expansion rate at their position from their observed radial velocities. Therefore, the precise measurement of distances is vital in this analysis.

To date, three editions of Cosmicflows have been published (Tully et al. 2008, 2013, 2016). Throughout, the samples are heterogeneous in that there is input involving multiple methodologies and sources. The scale foundations are set by the Cepheid period-luminosity relation (CPLR; Leavitt \& Pickering 1912) and the tip of the red giant branch (TRGB) standard candle (Lee et al. 1993), supplemented by methods that have high precision but limited applicability, such as detached eclipsing binaries (Pietrzyński et al. 2019) and the nuclear maser in NGC 4258 (Humphreys et al. 2013). These local calibrators constrain the scaling of methods useful at intermediate distances, such as the amplitudes of surface brightness fluctuations in early-type galaxies (Tonry \& Schneider 1988), the three-parameter fundamental plane correlation also applicable to early types (Djorgovski \& Davis 1987; Dressler et al. 1987), and the luminosity-rotation rate relation found to hold for spiral galaxies (Tully \& Fisher 1977). All of these procedures must be shown to be consistent with each other and, in turn, with the methodology that extends to large distances founded on the predictability of the peak luminosities of SNe Ia (Phillips 1993).

Cosmicflows-3 (CF3), the last edition reporting galaxy distances, contains information on 17,647 systems. $^{6}$ Roughly

\footnotetext{
6 The CF3 catalog updated with minor corrections is available at http://edd. ifa.hawaii.edu.
}

half the contributions in that compilation come from a single source: fundamental plane measurements from the Six Degree Field Redshift Survey (Magoulas et al. 2012; Springob et al. 2014). This contribution is restricted to $\delta \leqslant 0$, the celestial south. As a consequence, the coverage in CF3 is strongly tilted toward the south. It is a particular concern of the next addition, Cosmicflows-4, that this imbalance in sky coverage be redressed. This new edition will contain updates across a broad spectrum of inputs, but most of all, there will be a major enhancement in the number of spiral luminosity-rotation rate measurements made available as a consequence of the large number of neutral hydrogen line profile detections resulting from the Arecibo Legacy Fast ALFA Survey (ALFALFA; Haynes et al. 2018) covering the decl. range $0<\delta<+38$. Photometry for these galaxies is alternatively available from the Sloan Digital Sky Survey (SDSS; Alam et al. 2015) and the Wide-field Infrared Satellite Explorer (WISE; Wright et al. 2010). The present paper presents up-to-date calibrations of the luminosity-rotation rate correlations in the bands of these surveys.

Since its inception (Tully \& Fisher 1977; Aaronson et al. 1979), the luminosity-rotation rate relation (hereafter TFR) has been refined and reevaluated by many users. If the interest is in galaxy distances, a primary concern is the control of bias (Willick 1994). The current analysis makes use of the correlation fit with errors taken in line widths, a procedure that has been called the "inverse" TFR (ITFR). This procedure was initially discussed as a general principle by Schechter (1980) and specifically with relevance to the TFR by Tully (1988). The issue was discussed in detail in Section 3 of Tully \& Pierce (2000). The slope of the power-law fit of the ITFR nulls the bias to a first approximation. Residual bias can be taken into account with a small correction to be discussed. 
It deserves emphasis that a focus on the correlation with errors taken in line width, the distance-independent parameter, is desired if the primary goal is the measurement of distances, but it is not the appropriate procedure if the interest is a physical understanding of the relationship between luminosity and rotation in spiral galaxies. In the latter case, it is more informative to make a bivariate fit, taking into account errors affecting both line widths and magnitudes (Giovanelli et al. 1997; Masters et al. 2006; Ponomareva et al. 2017; Lelli et al. 2019).

We employ the inverse relation, since our interest is the measurement of galaxy distances. Recalibrations have been carried out in both optical and infrared bands in the course of the Cosmicflows program. Tully \& Courtois (2012) calibrated the TFR at the $I$ band using 267 spirals in 13 clusters. Aaronson et al. (1979) suggested the use of near-infrared bands to improve the TFR methodology, given the lesser dust obscuration in host galaxies. Furthermore, the light of old stars peaks at longer wave bands; therefore, near-infrared luminosities may better represent the baryonic mass of galaxies. With the motivation of reducing the scatter about the TFR and getting more accurate distances, Sorce et al. (2013) extended the calibration of the TFR toward the infrared using Spitzer $3.6 \mu \mathrm{m}$ Infrared Array Camera (IRAC) imaging for the same sample of galaxies as used by Tully \& Courtois (2012). Using the available $I$-band magnitudes, they also observed a correlation between the optical-infrared color index and the deviation of galaxy magnitudes from the infrared TFR. They found a tighter TFR after adjusting [3.6] mag for the effect of an $I-[3.6]$ color term.

Neill et al. (2014) followed the same procedure using the WISE (Wright et al. 2010) and added 43 galaxies to the previous sample. They also updated the calibration of the $I$ band TFR using their larger sample. They studied the possibility of decreasing the scatter about the infrared TFR by taking into account the optical-infrared color terms. Moreover, they showed that adopting a quadratic form for the TFR results in slightly smaller scatter about the relation.

In our program, we have performed the multiband photometry of $\sim 20,000$ Cosmicflows-4 candidate galaxies in optical (SDSS $u, g, r, i$, and $z$ ) and/or infrared WISE $W 1(3.4 \mu \mathrm{m})$ and $W 2(4.6 \mu \mathrm{m})$ bands. The purpose of the current study is to provide the calibration of the TFR at these wave bands to measure distances for our full sample of galaxies. To achieve our goal, we use a sample of 648 calibrator galaxies (roughly double that available previously) in 20 clusters (up from 13). We explore intrinsic relations between color, morphology, and other galaxy observables with deviations from the TFR at different wave bands toward possible ways to reduce scatter and hopefully obtain more accurate distances.

We explain our sample selection criteria and present our data product in Section 2. In Section 3, we calibrate the luminosityline width correlations. In Section 4, we explore the possibility of reducing the scatter of the calibrated relations using additional observables, such as color indices and surface brightnesses. The radial velocities of the calibrator clusters are determined in Section 5, and their measured distance moduli are evaluated across all passbands and calibrations in Section 6. We provide estimations of the Hubble constant, $H_{0}$, on the basis of the clusters in Section 7. Our conclusion is presented in Section 8 .

\section{Data}

\subsection{Calibrators}

To calibrate the luminosity-line width correlation in spirals, we follow the same methodology that was originally described by Tully \& Courtois (2012) and further implemented by Sorce et al. (2013) and Neill et al. (2014). In this technique, the TFR calibration is carried out using spirals that reside in galaxy clusters. In a cluster, all spirals are assumed to be at the same distance; therefore, the correlation of apparent luminosities with rotation rates is manifested. Weighted least-squares fits assume errors in line widths (ITFR). Considering individual clusters separately, the slopes are similar but the intercepts differ because the clusters are at different distances. Upon deriving the TFR slopes for all individual clusters, the initial step toward a universal template is taken by shifting the galaxies of each cluster along the luminosity axis to a fiducial distance (for convenience, that set by the Virgo cluster sample; the nearest case and populated across the fully useful magnitude range). In this first pass, the shift for a cluster is given by the magnitude difference with respect to the fiducial at the intercept magnitude value at a log line width equal to 2.5. The tentative universal template that has been formed, treating all of the galaxies as if belonging to the same cluster and at the same distance, has a more complete magnitude coverage and reduces the uncertainty of the calibration slope. The tentative universal slope is now forced in a least-squares sense onto all of the individual clusters. The intercept magnitude differentials with respect to the fiducial mandate the shifts to form a new universal relation. The procedure is repeated iteratively, rapidly reaching convergence.

It is to be remarked that the universal template that is constructed is decoupled from variations in the cosmic expansion. More typically in other studies, templates are formed by assuming the constituents, on average, participate in the Hubble flow (Willick et al. 1996; Giovanelli et al. 1997; Springob et al. 2007, 2014). We endeavor at every step to decouple the measurement of distances from velocity information, since a principal goal of our program is to identify departures from Hubble expansion.

To calibrate the zero-point of the TFR, we use a subset of nearby spirals with accurate distance measurements from independent methods, such as the TRGB and/or CPLR. In the case of availability of distance measurements from both methods, we use the average value. Details are provided in Section 3.2. The key point to mention is that the slope applied to the zero-point sample is accepted to be given by the universal cluster calibration. There is no sense of completion to any magnitude limit with the zero-point calibration sample. The error-weighted least-squares fit to the zero-point sample transforms the universal cluster template relation to an absolute magnitude scale.

The calibrator sample of this study is a subset of our large sample of $\sim 20,000$ candidates that we compiled to measure distances using the luminosity-line width method. ${ }^{7}$ Galaxies of this sample are restricted to having (1) high-quality $\mathrm{H} \mathrm{I}$ measurements (see Section 2.2), (2) morphological type Sa or later, and (3) spirals with inclinations greater than $45^{\circ}$ from face-on.

In this program, we measured the spiral inclinations by visually comparing them with a set of spirals with known

\footnotetext{
This catalog will be published in a separate paper.
} 
inclinations. Sorting spirals based on their inclinations was executed through an online graphical tool, Galaxy Inclination Zoo, ${ }^{8}$ as a participative science project with citizens. Comparisons with inclinations derived from axial ratios (Neill et al. 2014) indicate that the rms uncertainties are $\pm 4^{\circ}$. In detail, the uncertainties are $\pm 3^{\circ}$ more edge-on than $70^{\circ}$, degrading to $\pm 5^{\circ}$ by inclinations of $50^{\circ}$. Please refer to Section 2.5 of Kourkchi et al. (2019, hereafter K19) for further details.

We note that inclinations can be determined in an independent way from the projection of disk rotation with resolved velocity field information. The number of sources that have been given sufficient study are limited, since observations must be made with radio interferometers. The targets must be large and $\mathrm{HI}$-rich and warrant the investment of resources. Nonetheless, the studies that have been done provide the basis for a comparison. We draw on intersecting samples of 30 galaxies by Ponomareva et al. (2017) and 97 galaxies by Lelli et al. (2016). The kinematically determined inclinations in the two interferometric studies concur and are systematically more face-on than the inclinations from images by 3.3 with $4 \sigma$ significance. The scatter around this offset is \pm 4.8 . Hence, the scatter is satisfactory, suggesting statistical uncertainties at a level of $4^{\circ}$ for each method but revealing a systematic in at least one of the methods. The information necessary to provide kinematic inclinations is not available for the samples of thousands of galaxies in our study. A systematic offset would affect our calibrators and field targets alike. We do not detect any trend in measured distances as a function of inclination.

For our calibration, we select galaxy clusters that have substantial numbers of galaxies with the requisite line width and photometric information (median of 24 galaxies per cluster). General properties of clusters, such as center, $R_{2 t}$, $V_{c}$, and $\sigma_{p}$, are taken from a galaxy group catalog (Tully 2015) that is based on the Two Micron All Sky Survey (2MASS) Redshift Survey quasi-complete to $K_{s}=11.75$ (Huchra et al. 2012), where $R_{2 t}$ is the cluster projected second turnaround radius, $V_{c}$ is the average heliocentric radial velocity of the cluster, and $\sigma_{p}$ is the cluster projected velocity dispersion. A galaxy is included in a calibrator cluster if it meets one of the following conditions: (1) the galaxy projected distance from the center of the cluster, $R_{p}$, is less than $1.5 R_{2 t}$, and its heliocentric velocity is within $3 \sigma_{p}$ of $V_{c}$; or (2) the projected distance is relaxed to $1.5 R_{2 t} \leqslant R_{p}<3 R_{2 t}$, but the heliocentric velocity is more constrained at less than $2 \sigma_{p}$ of $V_{c}$.

Based on experience with earlier releases of Cosmicflows, as many as $3 \%$ of the galaxies selected for the TFR analysis do not follow the general trend of the TFR. The outlier galaxies could not be initially rejected based on their morphology or the quality of photometry and/or H I measurements and/or other observables that we use in this study. We exclude them from our sample as $>3 \sigma$ deviants when fitting the TFR. We label these galaxies as "anomalous galaxies" and distinguish them in our plots.

Our selection criteria leave us with 648 galaxies (including 13 anomalous galaxies) from our large sample as candidate members of our 20 clusters. $^{9}$ We use these galaxies for the slope calibration of the TFR. For the zero-point calibration,

\footnotetext{
8 http://edd.ifa.hawaii.edu/inclination/index.php

9 Galaxies with $\mathrm{H}$ I line widths less than $64 \mathrm{~km} \mathrm{~s}^{-1}$ are preemptively dropped because such galaxies will not survive our final absolute magnitude cut of $M_{i} \leqslant-17$.
}

there are 94 spirals that have distance measurements from the Cepheid luminosity period or TRGB methods.

There are a few confusing regions where clusters overlap in plane-of-sky projections. For example, in the case of the Virgo cluster, we exclude galaxies that have higher chances of being members of background galaxy groups. We include a galaxy in the Virgo cluster within its virial radius if its heliocentric velocity satisfies either $V_{h}<600 \mathrm{~km} \mathrm{~s}^{-1}$ or $1200 \mathrm{~km} \mathrm{~s}^{-1}$ $<V_{h}<1600 \mathrm{~km} \mathrm{~s}^{-1}$ to avoid galaxies that are potentially in overlapping groups: Virgo $\mathrm{W}$ and Virgo $\mathrm{M}$ at $V_{h}>1600$ $\mathrm{km} \mathrm{s}^{-1}$ and Virgo W' and Virgo W' in the window $600 \mathrm{~km} \mathrm{~s}^{-1}$ $<V_{h}<1200 \mathrm{~km} \mathrm{~s}^{-1}$. See Section 6.1 of Tully et al. (2016) for an extensive discussion of potential confusion. In another special case, the Ursa Major entity consists of seven smaller galaxy groups identified in Kourkchi \& Tully (2017) that are almost indistinguishably at the same distance along a filament that spans 7.5 across the plane of the sky.

\subsection{H I Line Widths}

One of the criteria for our sample selection is the existence of high-quality $\mathrm{H}$ I data. We take $\mathrm{H} \mathrm{I} 21 \mathrm{~cm}$ line widths and fluxes from various sources in the order of priority listed below.

1. The All Digital HI catalog (ADHI) that has been compiled in the course of the Cosmicflows program (Courtois et al. 2009, 2011), available at the Extragalactic Distance Database (EDD) website. ${ }^{10}$ Entries in this catalog contain the $\mathrm{H}$ I line width parameter $W_{m x}$, the parameter that encodes galaxy rotation rates at their negative and positive maximum intensities along the line of sight. Here $W_{m x}$ is calculated from the observed $W_{m 50}$ and adjusted for spectral resolution and redshift. $W_{m 50}$ is the line width at $50 \%$ of the average H I flux within the range that covers $90 \%$ of the total H I flux. Low signal-tonoise ratio $(\mathrm{S} / \mathrm{N})$ and confused or anomalous line profiles are cause for the rejection of potential candidates. We only accept ADHI $W_{m x}$ values if the evaluated uncertainties are less than or equal to $20 \mathrm{~km} \mathrm{~s}^{-1}$.

2. ALFALFA (Haynes et al. 2011, 2018). To be compatible with the ADHI values, cataloged ALFALFA line widths are adjusted using $W_{m x}=W_{\text {alf }}-6 \mathrm{~km} \mathrm{~s}^{-1}$, a formula derived for spirals with $\mathrm{H}$ I line width measurements in both catalogs. We require $\mathrm{S} / \mathrm{N}>10$ for line widths taken from ALFALFA, which is fairly consistent with the adopted uncertainty condition for an ADHI estimate.

3. The Springob/Cornell H I catalog (Springob et al. 2005). The $W_{M 50}$ values provided in this catalog are transformed using $W_{m 50}=1.015 W_{M 50}-11 \mathrm{~km} \mathrm{~s}^{-1}$ and then translated into $W_{m x}$ values using the ADHI standard formalism (Courtois et al. 2009).

4. The Pre Digital H I catalog available in EDD provides information from early analog H I line profiles (Fisher \& Tully 1981; Huchtmeier \& Richter 1989). Recourse to this old material is needed in some cases of nearby galaxies that are much larger than the beam sizes of large radio telescopes. This catalog lists $W_{20}$, the width at $20 \%$ of the H I profile peak. We convert $W_{20}$ to $W_{m x}$ based on the relation described by Courtois et al. (2009).

The parameter $W_{m x}$ is taken as a robust measure of galaxy rotation after correction for the effect of inclination. We

\footnotetext{
${ }^{10}$ http://edd.ifa.hawaii.edu; catalog “All Digital H I."
} 
introduce a minimum threshold for our potential candidates at $W_{m x}>64 \mathrm{~km} \mathrm{~s}^{-1}$, eliminating dwarf galaxies that, at any rate, would not survive our restriction later imposed of $M_{i}<-17$. Input from the Springob/Cornell and Pre Digital H I catalogs is not considered if a galaxy has high-quality $\mathrm{H}$ I measurements in either of the catalogs ADHI (uncertainty of $W_{m x}$ not larger than $\left.20 \mathrm{~km} \mathrm{~s}^{-1}\right)$ or ALFALFA $(\mathrm{S} / \mathrm{N}>10)$.

We take the average values of $\mathrm{HI}$ flux/line width for galaxies that are in both the ADHI and ALFALFA catalogs. If a galaxy is not tabulated in either the ADHI or ALFALFA catalogs, we extract HI measurements from either the Springob/Cornell or Pre Digital H I catalogs, with Springob/ Cornell having higher priority.

Considerable attention has been given in the past to studies of the resolved rotation characteristics of spirals toward the twin quests of reducing scatter in the TFR and gaining physical insight into causes of intrinsic scatter (Verheijen 2001; Noordermeer \& Verheijen 2007; Lelli et al. 2016, 2019; Ponomareva et al. 2016, 2017). These interferometric studies identify three classes: rotation curves that fall below their maximum rate at large radii, rotation curves that roughly maintain their maximum rates at large radii, and rotation curves that are continuing to rise at large radii. Massive and relatively early types tend to manifest themselves in the first of these classes, while galaxies of low intrinsic luminosity are found in the third class. Scatter in the TFR is minimized by characterizing rotation with the parameter $V_{\text {flat }}$, determined by the resolved rotation rate at the extremity of a galaxy (Ponomareva et al. 2017; Lelli et al. 2019). Rotation parameters constructed from the rotation rate at interior radii, such as $V_{2.2}$, at a radius of 2.2 scale lengths cause deterioration of the TFR. These studies are instructive, but global profiles from singlebeam radio observations integrate over the $\mathrm{H} \mathrm{I}$ flux of galaxies. It is encouraging that Lelli et al. (2019) found that global profile parameters perform only slightly less well than $V_{\text {flat }}$ and significantly better than parameters representative of rotation at inner radii. It can be gathered that much of the integrated H I flux from a galaxy arises from relatively large radii.

In addition to a line width, we derive an $\mathrm{HI} 21 \mathrm{~cm}$ magnitude, $m_{21}$, from the $\mathrm{H}$ I fluxes following

$$
m_{21}=-2.5 \log F_{\mathrm{HI}}+17.40
$$

where $F_{\mathrm{H} \text { I }}$ is the flux within the $21 \mathrm{~cm}$ line profile in units of $\mathrm{Jy} \mathrm{km} \mathrm{s}^{-1}$ (Makarov et al. 2014).

\subsection{Photometry}

Photometry for the calibrator galaxies is carried out on optical and infrared images provided by the SDSS DR12 and WISE surveys. The process of obtaining individual exposures, image preprocessing, and performing photometry was described in detail in Sections 2.2-2.4 of K19. Our asymptotic magnitudes are calculated within the aperture radius at the point where the cumulative luminosity curve of growth flattens. For comparison, we computed total magnitudes with extrapolations assuming exponential disks following Tully et al. (1996), finding agreement with a scatter of 0.02 mag. Uncertainties in magnitudes are $0.05 \mathrm{mag}$, dominated by the setting of the sky background.

Out of 648 candidate calibrator galaxies, 464 spirals (eight anomalous) have SDSS photometry at the $u, g, r, i$, and $z$ bands. For 600 (19 anomalous) galaxies, we make use of WISE
W1- and W2-band coverage. There is both optical and infrared photometry coverage for 435 galaxies.

The observed apparent magnitude at each wave band, $m_{\lambda}^{\text {total }}$, is adjusted using the following relation:

$$
m_{\lambda}^{*}=m_{\lambda}^{\text {(total) }}-A_{b}^{\lambda}-A_{k}^{\lambda}-A_{a}^{\lambda}-A_{i}^{\lambda},
$$

where $A_{b}^{\lambda}$ is the Milky Way dust extinction, $A_{k}^{\lambda}$ is the correction for the redshift effect on the galaxy luminosity at each passband ( $k$-correction), and $A_{a}^{\lambda}$ is the total flux aperture correction for the photometry of the extended objects when the photometry calibration was performed using point-source objects (stars). There is a full discussion of the calculation of $A_{b}^{\lambda}, A_{k}^{\lambda}$, and $A_{a}^{\lambda}$ in Section 2.5 of K19.

Here $A_{i}^{\lambda}$ is the dust attenuation within the host galaxy that depends on (1) the galaxy physical properties, modeled as a function of galaxy observables, and (2) the galaxy spatial orientation with respect to the observer (K19). At each wave band $\lambda, A_{i}^{\lambda}$ is modeled as

$$
A_{i}^{\lambda}=\gamma_{\lambda} \mathcal{F}_{\lambda}(i)
$$

where $\mathcal{F}_{\lambda}(i)$ is a function of galaxy inclination, $i,{ }^{11}$ and $\gamma_{\lambda}$ is a third-degree polynomial function of the main principal component, $P_{1, \mathrm{w} 2}$, that is constructed from the linear combination of galaxy observables using

$$
\begin{aligned}
P_{1, \mathrm{~W} 2}= & 0.524\left(\log W_{m x}^{i}-2.47\right) / 0.18 \\
& +0.601\left(C_{21 \mathrm{~W} 2}-1.63\right) / 1.15 \\
& -0.603\left(\left\langle\mu_{2}\right\rangle_{e}^{(i)}-23.35\right) / 1.38,
\end{aligned}
$$

where $W_{m x}^{i}$ is the $\mathrm{HI}$ line width corrected for inclination following $W_{m x}^{i}=W_{m x} / \sin (i), C_{21 \mathrm{~W} 2}=m_{21}-\bar{W} 2$ is a pseudocolor calculated from the difference between the $\mathrm{HI} 21 \mathrm{~cm}$ and W2 magnitudes, and $\left\langle\mu_{2}\right\rangle_{e}^{(i)}$ is the average surface brightness of the galaxy at the $W 2$ band within the effective radius that is corrected for the geometric effect of inclination given by

$$
\left\langle\mu_{2}\right\rangle_{e}^{(i)}=\left\langle\mu_{2}\right\rangle_{e}+0.5 \log _{10}(a / b),
$$

where $a$ and $b$ are the semimajor and semiminor axes of the photometry aperture. Given the effective radius of a galaxy, $R_{\lambda e}$, its effective surface brightness is

$$
\left\langle\mu_{\lambda}\right\rangle_{e}=m_{\lambda}^{(\text {total })}+2.5 \log _{10}\left(2 \pi R_{\lambda e}^{2}\right) .
$$

There is a full discussion in K19 regarding the calculation of $A_{i}^{\lambda}$.

The above formalism for calculating the dust attenuation of host galaxies relies on the existence of infrared photometry. However, there are 29 spirals in our sample of TFR calibrators for which we lack infrared photometry. We use a prediction algorithm to estimate their internal dust attenuation, $A_{i}^{\lambda}$, from their optical photometry and $21 \mathrm{~cm}$ line widths/fluxes.

In concept, given the luminosity of a galaxy at optical wave bands and some other information about its physical properties, such as intrinsic size and/or morphology, one can predict the galaxy luminosity at longer wave bands. One common method is to fit the spectral energy distribution (SED) over the observed magnitudes using a set of template SEDs that represent the

\footnotetext{
11 Symbol $i$ not to be confused with the photometric passband.
} 
morphology, size, and physical properties of the sample galaxies (Du et al. 2020).

In this study, we use a less complicated prediction scheme. We orchestrate a random forest algorithm together with a set of distance-independent observables to predict the missing infrared information. Our prediction algorithm is trained using 2000 spirals with full optical/infrared magnitude coverage. The trained algorithm is capable of predicting W2 magnitudes with an rms uncertainty of $\sim 0.2 \mathrm{mag}$. Based on the predicted $W 2$ magnitudes, the $1 \sigma$ uncertainty of the predicted $\gamma_{\lambda}$ is $\sim 0.04$ mag for the optical bands and smaller at longer wavelengths. Then $\gamma_{\lambda}$ is multiplied by $\mathcal{F}_{\lambda}$ to obtain the dust attenuation $A_{i}^{\lambda}$. Here $\mathcal{F}_{\lambda}$ is a monotonic increasing function of inclination that is maximal for fully edge-on galaxies between 1.5 and 1.75 for the optical wave bands and 0.75 for the $W 1$ band (K19). The overall uncertainty on our predicted $A_{i}^{\lambda}$ values is always not worse than $\sim 0.07 \mathrm{mag}$. We leave further details of this procedure for a later paper (E. Kourkchi et al. 2020, in preparation).

\subsection{Data Catalog}

The measurements and collection of parameters used in this study are collected in Table 1 for 648 cluster galaxies and 94 zero-point calibrators. Column contents are (1) Principal Galaxies Catalog (PGC) number; (2) common name; (3) measured inclination angle of the galaxy in degrees; (4) H I line width in $\mathrm{km} \mathrm{s}^{-1}$; (5) logarithm of the inclination-corrected H I line width calculated from $W_{m x}^{i}=W_{m x} / \sin (i)$, where $i$ is the inclination angle presented in column (3); (6) $\mathrm{H} \mathrm{I} 21 \mathrm{~cm}$ magnitude calculated from the $\mathrm{HI}$ flux, $F_{\mathrm{HI}}$, using $m_{21}=$ $-2.5 \log F_{\mathrm{H} \mathrm{I}}+17.4$; (7)-(11) $\operatorname{SDSS} u, g, r, i$, and $z$ total raw magnitudes in the AB system; (12)-(13) WISE $W 1$ and $W 2$ total raw magnitudes in the $\mathrm{AB}$ system; ${ }^{12}$ (14) $b / a$ axial ratios of the elliptical photometry apertures used for the photometry of SDSS images, where $a$ and $b$ are the semimajor and semiminor axes of the elliptical aperture, respectively; (15) analogous to column (14) but for the photometry apertures of the WISE images; (16)-(22) semimajor axes of apertures that enclose half the total light of galaxies at optical/infrared bands in arcminutes; (23) name of the host cluster, with the 94 zeropoint calibrators identified as " $\mathrm{ZP}$ calibrator" and an asterisk added to the end of the cluster name if a galaxy is labeled as "anomalous," i.e., strongly deviant for no reason that is evident; (24)-(30) tabulated optical/infrared magnitudes corrected for Milky Way obscuration, redshift $k$-correction, and aperture effects, but not the inclination-dependent dust attenuation, based on the corresponding raw magnitudes listed in columns (7)-(13); (31)-(37) dust attenuation corrections at the optical/infrared band, calculated from Equation (3); and (38)-(44) correspondence to columns (31)-(37) after correcting for the effect of global dust obscuration, $m_{\lambda}^{*}=$ $m_{\lambda}^{(\text {total })}-A_{b}^{\lambda}-A_{k}^{\lambda}-A_{a}^{\lambda}-A_{i}^{\lambda}$.

\section{Calibration of the TFR}

We follow the same ITFR fitting procedure as explained in Tully \& Courtois (2012) to calibrate the TFR for all wave bands of interest. Accordingly, the errors and residuals in $\mathrm{H} \mathrm{I}$

\footnotetext{
12 The uncertainties on the measured $u$-band magnitudes are $\sim 0.1 \mathrm{mag}$. The uncertainties on all other measured magnitudes are not worse than $0.05 \mathrm{mag}$, which is conservatively adopted for the purpose of error propagation in this study.
}

line width are taken into account in the linear least-squares regression. Bias errors are reduced by a factor of 5 with respect to a direct TFR analysis (Willick 1994), reducing the corrections required to account for bias from a serious to a minor problem. The major bias with use of the direct relation arises because at a given line width, an intrinsically brighter galaxy drawn from above the mean relation is prone to be included in a sample, while an intrinsically fainter galaxy may be lost. With the inverse relation, galaxies of a given magnitude with wider and narrower line widths are equally likely to be included. See the discussion in Section 1 and Tully \& Pierce (2000).

In this study, the errors of the measured magnitudes at all wave bands are much smaller than the uncertainties in the measured line widths. Thus, we only use line width errors as the input measurement error. Although the inverse relation mitigates the Malmquist selection bias, the scatter in the sample induces a residual bias, which is addressed in Section 3.6.

\subsection{The ITFR Template Slope}

There is the fundamental assumption in using the luminosity-rotation rate correlation as a tool for measuring distances that galaxies in diverse environments statistically follow a common relationship. This assumption can be evaluated by giving attention to meaningfully large and varied samples. Drawing upon individual galaxy clusters has the useful feature that all candidates are supposed to be at the same distance. Samples can be constructed that are quasi-complete to apparent magnitude limits, with exclusions because of face-on orientation, confusion, or morphological anomalies that are expected to be randomly drawn from the mean correlation. Clusters do vary in their richness and the relative proportions of early- and late-type systems. It can be asked if spirals in clusters have the same properties as spirals outside of clusters. There is remarkably little evidence that there are differences in ways that affect the TFR. Plausibly, gas-rich spirals in clusters of all sorts are recent arrivals that retain their pre-entry properties. The issue deserves continual scrutiny. Our 20 clusters are wideranging in their properties, from dense and populous to diffuse and representative of the field. As will be shown, the 20 clusters share the commonalities of correlation slopes and scatter, supportive of the proposition of universal relations between galaxy luminosities and rotation rates.

Assuming that there is a universal TFR describable by a power law, then a key property of ITFR fits allows us to combine multiple cluster samples to form a robust template. The slope of the ITFR is independent of the depth of the magnitude limit, within statistical uncertainties. By contrast, the slope of the direct TFR becomes shallower as the magnitude limit is raised. A fit that combines errors on both axes shares the same property in a lesser fashion. This unique property of the ITFR of the decoupling of the slope from the depth of coverage allows cluster samples to be slid on top of each other with simple shifts in magnitude. Nearby clusters will sample to the faintest magnitudes of applicable interest. Distant clusters will only populate the upper part of the correlation, but do so drawing from the same slope. Of course, to be useful, each individual sample must be sufficiently numerous as to allow for an independent determination of slope to judge consistency and merge with the ensemble with meaningful statistical uncertainty. 
Table 1

Data Catalog

\begin{tabular}{|c|c|c|c|c|c|c|c|c|c|c|c|c|c|c|c|c|c|c|c|c|c|}
\hline PGC & $\begin{array}{l}\text { Name } \\
\text { (2) }\end{array}$ & $\begin{array}{l}\text { Inc. } \\
\text { (deg) } \\
\text { (3) }\end{array}$ & $\begin{array}{c}W_{m x} \\
\left(\mathrm{~km} \mathrm{~s}^{-1}\right) \\
(4)\end{array}$ & $\begin{array}{c}\log \left(W_{m x}^{i}\right) \\
(5)\end{array}$ & $\begin{array}{c}m_{21} \\
(\mathrm{mag}) \\
(6)\end{array}$ & $\begin{array}{c}u \\
\text { (mag) } \\
(7)\end{array}$ & $\begin{array}{c}g \\
\underset{(\mathrm{mag})}{(8)}\end{array}$ & $\begin{array}{c}r \\
(\mathrm{mag}) \\
(9)\end{array}$ & $\begin{array}{c}i \\
(\mathrm{mag}) \\
(10)\end{array}$ & $\begin{array}{c}z \\
(\mathrm{mag}) \\
(11)\end{array}$ & $\begin{array}{c}W 1 \\
(\mathrm{mag}) \\
(12)\end{array}$ & $\begin{array}{c}W 2 \\
(\mathrm{mag}) \\
(13)\end{array}$ & $\begin{array}{c}(b / a)_{S} \\
(14)\end{array}$ & $\begin{array}{c}(b / a)_{W} \\
(15)\end{array}$ & $\begin{array}{c}R_{e u} \\
(\operatorname{arcmin}) \\
(16)\end{array}$ & $\begin{array}{c}R_{e g} \\
(\operatorname{arcmin}) \\
(17)\end{array}$ & $\begin{array}{c}R_{e r} \\
(\operatorname{arcmin}) \\
(18)\end{array}$ & $\begin{array}{c}R_{e i} \\
(\operatorname{arcmin}) \\
(19)\end{array}$ & $\begin{array}{c}R_{e z} \\
(\operatorname{arcmin}) \\
(20)\end{array}$ & $\begin{array}{c}R_{e W 1} \\
(\operatorname{arcmin}) \\
(21)\end{array}$ & $\begin{array}{c}R_{e W 2} \\
\text { (arcmin) } \\
(22)\end{array}$ \\
\hline 38803 & IC 3033 & $59 \pm 5$ & $111 \pm 1$ & $2.112 \pm 0.023$ & $15.811 \pm 0.076$ & 15.89 & 14.93 & 14.55 & 14.34 & 14.31 & 14.91 & 15.21 & 0.46 & 0.53 & 0.36 & 0.35 & 0.33 & 0.33 & 0.31 & 0.38 & 0.49 \\
\hline 38943 & NGC 4178 & $75 \pm 3$ & $249 \pm 2$ & $2.410 \pm 0.007$ & $12.884 \pm 0.076$ & 12.43 & 11.68 & 11.21 & 10.92 & 10.83 & 11.31 & 11.88 & 0.35 & 0.34 & 1.59 & 1.38 & 1.34 & 1.34 & 1.28 & 1.21 & 1.22 \\
\hline 38945 & IC 3044 & $73 \pm 3$ & $125 \pm 3$ & $2.116 \pm 0.012$ & $14.975 \pm 0.076$ & 15.02 & 14.02 & 13.62 & 13.41 & 13.31 & 14.04 & 14.77 & 0.36 & 0.40 & 0.52 & 0.54 & 0.56 & 0.57 & 0.58 & 0.61 & 0.57 \\
\hline 39028 & NGC 4192 & $78 \pm 3$ & $458 \pm 2$ & $2.671 \pm 0.005$ & $12.724 \pm 0.076$ & 11.57 & 10.49 & 9.77 & 9.36 & 9.13 & 9.38 & 9.98 & 0.24 & 0.36 & 2.76 & 1.96 & 1.82 & 1.77 & 1.71 & 1.39 & 1.40 \\
\hline 39181 & IC 3066 & $83 \pm 3$ & $132 \pm 8$ & $2.123 \pm 0.025$ & $16.958 \pm 0.076$ & 16.70 & 15.38 & 14.84 & 14.57 & 14.44 & 15.14 & 15.79 & 0.33 & 0.33 & 0.22 & 0.25 & 0.25 & 0.26 & 0.26 & 0.29 & 0.29 \\
\hline 39224 & NGC 4212 & $53 \pm 4$ & $252 \pm 1$ & $2.499 \pm 0.023$ & $14.734 \pm 0.076$ & 12.71 & 11.51 & 10.90 & 10.57 & 10.39 & 10.67 & 11.20 & 0.70 & 0.74 & 0.59 & 0.59 & 0.58 & 0.58 & 0.56 & 0.56 & 0.56 \\
\hline 39246 & NGC 4216 & $84 \pm 3$ & $511 \pm 1$ & $2.711 \pm 0.003$ & $13.752 \pm 0.076$ & 12.11 & 10.48 & 9.64 & 9.15 & 8.91 & 9.04 & 9.69 & 0.24 & 0.32 & 1.76 & 1.43 & 1.27 & 1.23 & 1.13 & 1.00 & 1.04 \\
\hline 39256 & IC 3077 & $58 \pm 4$ & $58 \pm 13$ & $1.835 \pm 0.102$ & $18.110 \pm 0.076$ & 15.41 & 14.16 & 13.54 & 13.16 & 13.07 & 13.72 & 14.71 & 0.65 & 0.72 & 0.59 & 0.58 & 0.56 & 0.58 & 0.56 & 0.54 & 0.42 \\
\hline 39308 & NGC 4222 & $90 \pm 1$ & $220 \pm 1$ & $2.342 \pm 0.002$ & $14.293 \pm 0.076$ & 14.68 & 13.54 & 12.90 & 12.53 & 12.35 & 12.43 & 12.94 & 0.14 & 0.32 & 0.89 & 0.85 & 0.83 & 0.83 & 0.77 & 0.52 & 0.52 \\
\hline 39431 & IC 3105 & $84 \pm 3$ & $83 \pm 2$ & $1.922 \pm 0.010$ & $15.129 \pm 0.076$ & 15.21 & 14.57 & 14.35 & 14.28 & 14.15 & 15.12 & 16.00 & 0.23 & 0.42 & 0.51 & 0.51 & 0.52 & 0.52 & 0.54 & 0.44 & 0.43 \\
\hline
\end{tabular}

\begin{tabular}{|c|c|c|c|c|c|c|c|c|c|c|c|c|c|c|c|c|c|c|c|c|c|c|}
\hline PGC & $\begin{array}{l}\text { Cluster } \\
\text { (23) }\end{array}$ & $\begin{array}{c}\bar{u} \\
\text { (mag) } \\
(24)\end{array}$ & $\begin{array}{c}\bar{g} \\
\text { (mag) } \\
(25)\end{array}$ & $\begin{array}{c}\bar{r} \\
(\mathrm{mag}) \\
(26)\end{array}$ & $\begin{array}{c}\bar{i} \\
(\mathrm{mag}) \\
(27)\end{array}$ & $\begin{array}{c}\bar{z} \\
(\mathrm{mag}) \\
(28)\end{array}$ & $\begin{array}{c}\overline{W 1} \\
(\mathrm{mag}) \\
(29)\end{array}$ & $\begin{array}{c}\overline{W 2} \\
(\mathrm{mag}) \\
(30)\end{array}$ & $\begin{array}{c}A_{u}^{(i)} \\
(\mathrm{mag}) \\
(31)\end{array}$ & $\begin{array}{c}A_{g}^{(i)} \\
(\mathrm{mag}) \\
(32)\end{array}$ & $\begin{array}{c}A_{r}^{(i)} \\
(\mathrm{mag}) \\
(33)\end{array}$ & $\begin{array}{c}A_{i}^{(i)} \\
(\mathrm{mag}) \\
(34)\end{array}$ & $\begin{array}{c}A_{z}^{(i)} \\
(\mathrm{mag}) \\
(35)\end{array}$ & $\begin{array}{c}A_{W 1}^{(i)} \\
\text { (mag) } \\
(36)\end{array}$ & $\begin{array}{c}A_{W 2}^{(i)} \\
\text { (mag) } \\
(37)\end{array}$ & $\begin{array}{c}u \\
\text { (mag) } \\
(38)\end{array}$ & $\begin{array}{c}g^{*} \\
(\mathrm{mag}) \\
(39)\end{array}$ & $\begin{array}{c}r^{*} \\
(\mathrm{mag}) \\
(40)\end{array}$ & $\begin{array}{c}i^{*} \\
(\mathrm{mag}) \\
(41)\end{array}$ & $\begin{array}{c}z \\
(\mathrm{mag}) \\
(42)\end{array}$ & $\begin{array}{c}W 1^{*} \\
(\mathrm{mag}) \\
(43)\end{array}$ & $\begin{array}{c}W 2^{*} \\
(\mathrm{mag}) \\
(44)\end{array}$ \\
\hline 38803 & Virgo & 15.75 & 14.83 & 14.48 & 14.29 & 14.27 & 14.91 & 15.21 & 0.24 & 0.16 & 0.11 & 0.09 & 0.07 & 0.01 & 0.00 & 15.51 & 14.67 & 14.37 & 14.20 & 14.20 & 14.90 & 15.21 \\
\hline 38943 & Virgo & 12.30 & 11.59 & 11.14 & 10.87 & 10.79 & 11.31 & 11.88 & 0.62 & 0.45 & 0.34 & 0.29 & 0.24 & 0.03 & 0.01 & 11.68 & 11.14 & 10.80 & 10.58 & 10.55 & 11.28 & 11.87 \\
\hline 38945 & Virgo & 14.87 & 13.90 & 13.54 & 13.35 & 13.26 & 14.03 & 14.76 & 0.43 & 0.27 & 0.19 & 0.16 & 0.12 & 0.00 & 0.00 & 14.44 & 13.63 & 13.35 & 13.19 & 13.14 & 14.03 & 14.76 \\
\hline 39028 & Virgo & 11.42 & 10.38 & 9.69 & 9.30 & 9.09 & 9.37 & 9.97 & 0.80 & 0.57 & 0.43 & 0.34 & 0.25 & 0.04 & 0.01 & 10.62 & 9.81 & 9.26 & 8.96 & 8.84 & 9.33 & 9.96 \\
\hline 39181 & Virgo & 16.56 & 15.28 & 14.77 & 14.52 & 14.40 & 15.14 & 15.79 & 0.79 & 0.53 & 0.39 & 0.32 & 0.26 & 0.02 & 0.01 & 15.77 & 14.75 & 14.38 & 14.20 & 14.14 & 15.12 & 15.78 \\
\hline 39224 & Virgo & 12.57 & 11.40 & 10.82 & 10.51 & 10.35 & 10.66 & 11.19 & 0.23 & 0.15 & 0.11 & 0.07 & 0.04 & 0.01 & 0.00 & 12.34 & 11.25 & 10.71 & 10.44 & 10.31 & 10.65 & 11.19 \\
\hline 39246 & Virgo & 11.97 & 10.37 & 9.57 & 9.10 & 8.87 & 9.03 & 9.69 & 0.81 & 0.45 & 0.29 & 0.13 & 0.00 & 0.01 & 0.01 & 11.16 & 9.92 & 9.28 & 8.97 & 8.87 & 9.02 & 9.68 \\
\hline 39256 & Virgo & 15.24 & 14.04 & 13.46 & 13.10 & 13.03 & 13.72 & 14.72 & 0.28 & 0.20 & 0.15 & 0.13 & 0.11 & 0.01 & 0.01 & 14.96 & 13.84 & 13.31 & 12.97 & 12.92 & 13.71 & 14.71 \\
\hline 39308 & Virgo & 14.54 & 13.44 & 12.83 & 12.48 & 12.31 & 12.43 & 12.94 & 1.64 & 1.26 & 1.02 & 0.88 & 0.75 & 0.05 & 0.02 & 12.90 & 12.18 & 11.81 & 11.60 & 11.56 & 12.38 & 12.92 \\
\hline 39431 & Virgo & 15.07 & 14.46 & 14.28 & 14.22 & 14.11 & 15.11 & 15.99 & 0.74 & 0.47 & 0.32 & 0.26 & 0.21 & 0.01 & 0.00 & 14.33 & 13.99 & 13.96 & 13.96 & 13.90 & 15.10 & 15.99 \\
\hline
\end{tabular}

(This table is available in its entirety in machine-readable form.) 
The algorithm to calibrate the slope of the ITFR is as follows. (1) The ITFR is fit independently to each individual cluster, given the apparent magnitudes and $\mathrm{H}$ I line widths of its constituent galaxies. (2) One cluster is chosen as a reference, and the other clusters are moved to nominally the same distance by shifting data along the magnitude axis based on the relative estimated zero-points of the individually fitted ITFRs. (3) Adopting the shifted magnitudes, the combined data of all translated clusters and the reference cluster form a tentative universal template. (4) The slope of the fitted ITFR for the combined sample of clusters (the universal slope) is fitted individually to the separate clusters. (5) We cycle back to step 2, updating iteratively following the same process until convergence. In the iterations at step 4, the newly revised universal slope is adopted, and with each cycle, the individual zero-points quickly settle to optimal values.

The estimated relative distances from the preliminary individual zero-points confirm that Virgo, Ursa Major, and Fornax are the closest clusters of our sample, and therefore they have the most complete magnitude coverage. To minimize the incompleteness effect in the farther clusters, we first combine the Virgo, Ursa Major, and Fornax clusters following the above algorithm. We adopt Virgo, the slightly nearer and most populated cluster, as the reference cluster. The universal slope is then updated iteratively by combining farther clusters in groups that are sorted based on their preliminary estimated relative distances. From closest to farthest, these cluster sets are (1) Centaurus, Antlia, and Pegasus; (2) Hydra, A262, NGC 410, NGC 507, and Cancer; (3) NGC 80, NGC 70, A1367, Coma, and A400; (4) A2634/66 and A539; and (5) A2151 (Hercules). These groups of clusters are added step-bystep to form our ultimate universal ensemble. In each step, the ITFR slope of the composed ensemble in the previous cycle is adopted as a fixed parameter to derive the relative distances of the remaining clusters. It is shown that this procedure works because the slope of the ITFR does not vary with the level of magnitude truncation in each cluster (Tully \& Courtois 2012; Sorce et al. 2013; Neill et al. 2014). This assertion is substantiated with Figure 1. Closer clusters to the left in the plots sample to fainter magnitudes of the ITFR.

Table 2 provides the universal slopes derived at different bands. Column (1) identifies the sequence of SDSS and WISE passbands. Column (2) gives the assigned codes for the resulting relations. Throughout the paper, $\mathrm{TF}_{\lambda}$ stands for the ITFR that is constructed based on the magnitudes given by Equation (2) and no further corrections. Column (3) identifies the nature of the sample; OP and IR stand for the samples with optical and infrared photometry measurements, respectively. Column (4) lists the number of galaxies that are involved in the ensemble when deriving the universal slope. Fewer galaxies with optical photometry are available than infrared because of the restricted sky coverage of SDSS. Column (5) records the universal slope. Column (6) provides the rms of magnitude deviations from the fitted ITFR averaged over the full applicable magnitude domain. In detail, scatter increases toward fainter magnitudes as discussed in Section 3.5. Columns (7)-(9) contain the zero-point information discussed in Section 3.2. Column (10) lists the adjustment on the ITFR zero-point discussed in Section 3.4.

As an example, Figure 2 displays the apparent magnitudes versus line widths for galaxies in the Virgo cluster at the SDSS $u, g, r, i$, and $z$ and WISE $W 1$ and $W 2$ passbands. In each panel, the dashed line is the least-squares fit to the filled colored points. In the fitting process, we exclude the outlier galaxies NGC 4424 (PGC 40809) and IC 3446 (PGC 41440) and galaxies with absolute SDSS $i$-band magnitudes fainter than -17 mag (see Section 3.2 regarding the absolute magnitude limit). In Figure 2, the excluded galaxies are represented by open gray points. The universal ITFR slopes, fit with the zeropoint as the only free parameter, are represented by the solid lines.

There are 14 clusters in our study with optical imaging coverage (the OP sample). Figure 3 illustrates the individual and universal ITFRs in the $i$ band for these clusters. Galaxies that are considered anomalous are displayed in red and excluded from the least-squares fits. The slopes of the solid lines in all panels of this figure are the same and equal to the universal slope of the ITFR in the $i$ band of $-8.32 \pm 0.13$. Complete information on the fitted relations is presented in Table 3.

The all-sky coverage of the WISE imaging enables us to consider more clusters in infrared bands. One exceptional case is A262, which is located at the edge of the SDSS survey such that there are only seven galaxies with SDSS photometry but 55 with WISE photometry. Figure 4 displays ITFRs for 20 clusters studied in the $W 1$ band, and Table 4 lists the parameters of the fits.

Our resulting universal ensembles in the $i$ and $W 1$ bands are illustrated in the two panels of Figure 5. The annotations list cluster names and zero-point offsets relative to the Virgo cluster. In cases of clusters with both optical and infrared coverage, the magnitude offsets at the $i$ and $W 1$ bands are in reasonable agreement.

\subsection{Zero-point Calibration and Absolute Distances}

We consider 94 galaxies that have distance estimates derived from either the CPLR or the TRGB luminosity feature. Not all of these are useful, particularly those that pertain to systems fainter than our ultimate $M_{i}=17$ cutoff, but there are many more potential zero-point calibrators than previously available. The absolute calibration of CF3 was based on 33 galaxies: 25 CPLR and 19 TRGB, with 11 in common. Here, as in the earlier study, the CPLR and TRGB calibration distances are found through completely separate pathways. The two resultant scales can be compared, evaluated, and combined as deemed appropriate.

In CF3, the CPLR calibration assumed a Large Magellanic Cloud (LMC) distance modulus of 18.50. Here we adopt a small change. Our zero-point scale is set to be consistent with that of Riess et al. (2019), which combines linkages with the LMC assumed at a modulus of $\mu_{\mathrm{LMC}}=18.477$ (Pietrzyński et al. 2019), the maser galaxy NGC 4258 at a modulus of $\mu_{N 4258}=29.40$ (Humphreys et al. 2013), and a Milky Waybased calibration. That paper argues for a small modification (a decrease in moduli of $-0.024 \mathrm{mag}$ ) of the CPLR distance moduli reported by Riess et al. (2016). In addition, three other sources of distances from the CPLR are incorporated. Groundbased observations provide measurements for very nearby galaxies. We average over two sources: those by Bhardwaj et al. (2016) and the Araucaria collaboration (Gieren et al. 2013; Zgirski et al. 2017) and related references. The two sources agree to within $0.02 \mathrm{mag}$ ( $1 \sigma$ with scatter $\pm 0.06 \mathrm{mag}$ ) and are given equal weight in averaging. Zero-points tied to the LMC are rescaled to agree with Riess et al. (2019). The other 
Table 2

The Calibrated Slope and Zero-point of the ITFRs at Different SDSS and WISE Passbands

\begin{tabular}{|c|c|c|c|c|c|c|c|c|c|}
\hline \multirow{2}{*}{$\begin{array}{l}\text { Band } \\
\text { (1) }\end{array}$} & \multirow{2}{*}{$\begin{array}{l}\text { TFR } \\
\text { Code } \\
\text { (2) }\end{array}$} & \multirow{2}{*}{$\begin{array}{c}\text { Sample } \\
\text { (3) }\end{array}$} & \multicolumn{3}{|c|}{ Universal Slope } & \multicolumn{3}{|c|}{ Zero-point } & \multirow{2}{*}{$\begin{array}{c}\text { ZP Adjustmen } \\
C_{\mathrm{zp}} \\
(10)\end{array}$} \\
\hline & & & $\begin{array}{l}\text { Ngal } \\
\text { (4) }\end{array}$ & $\begin{array}{l}\text { Slope } \\
\text { (5) }\end{array}$ & $\begin{array}{c}\mathrm{rms} \\
(6)\end{array}$ & $\begin{array}{l}\text { Ngal } \\
\text { (7) }\end{array}$ & $\begin{array}{l}Z P \\
(8)\end{array}$ & $\begin{array}{c}\mathrm{rms} \\
(9)\end{array}$ & \\
\hline$g$ & $\mathrm{TF}_{g}$ & $\mathrm{OP}$ & 430 & $-7.37 \pm 0.13$ & 0.49 & 39 & $-20.15 \pm 0.11$ & 0.62 & $-0.11 \pm 0.18$ \\
\hline$r$ & $\mathrm{TF}_{r}$ & OP & 430 & $-7.96 \pm 0.13$ & 0.49 & 39 & $-20.57 \pm 0.10$ & 0.59 & $-0.08 \pm 0.13$ \\
\hline$i$ & $\mathrm{TF}_{i}$ & OP & 430 & $-8.32 \pm 0.13$ & 0.49 & 39 & $-20.80 \pm 0.10$ & 0.59 & $-0.04 \pm 0.10$ \\
\hline$z$ & $\mathrm{TF}_{z}$ & $\mathrm{OP}$ & 429 & $-8.46 \pm 0.13$ & 0.50 & 38 & $-20.89 \pm 0.10$ & 0.57 & $-0.08 \pm 0.11$ \\
\hline
\end{tabular}
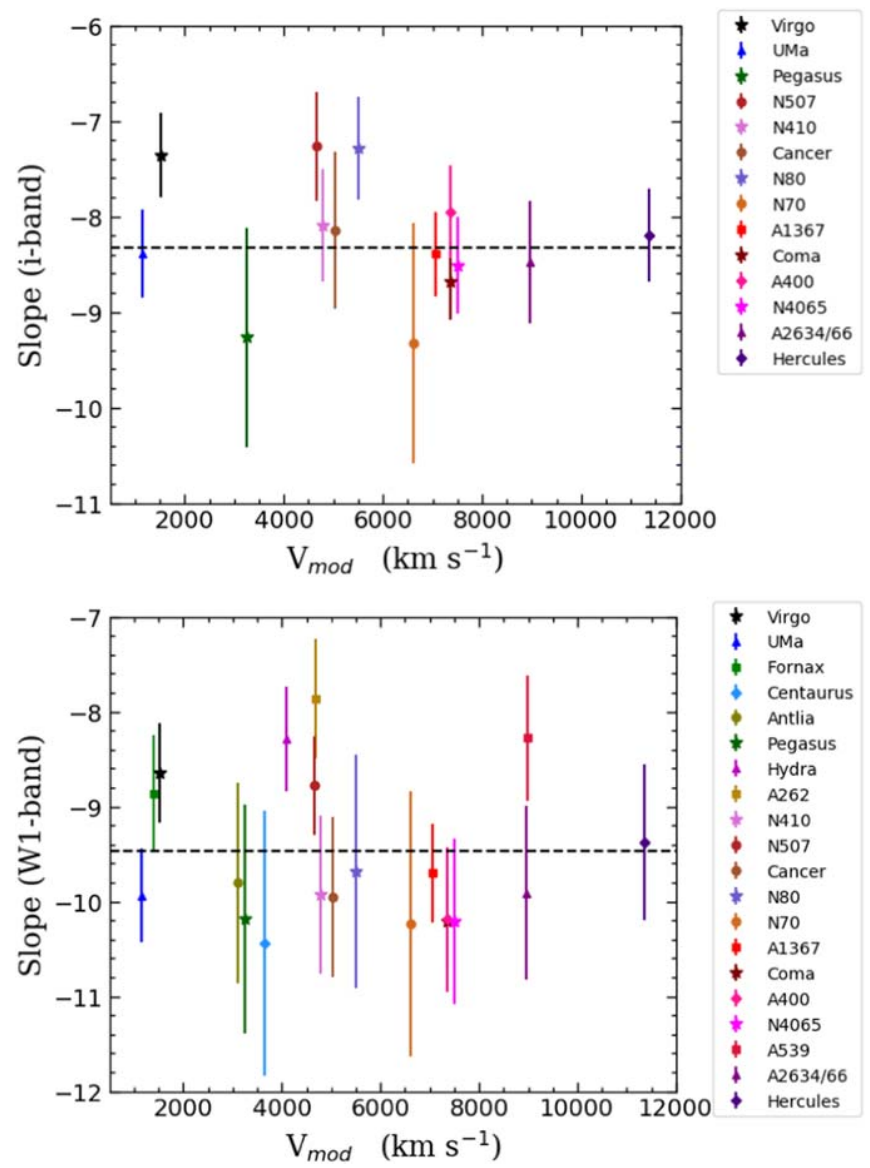

Figure 1. The ITFR slopes with standard deviations for individual clusters vs. systemic velocity. Top panel: $i$ band; bottom panel: $W 1$ band. Horizontal dashed lines are drawn at the level of the universal slopes. The legends on the right provide the cluster codes, listed in Tables 3 and 4.

Cepheid contribution is Freedman et al. (2001). The overlap with Riess et al. (2016) is only four objects, but the agreement is within 0.02 mag at the same LMC fiducial distance. Each of these inputs is rescaled to be consistent with Riess et al. (2019).

Our primary source for TRGB distances is the colormagnitude diagram/TRGB catalog in the $\mathrm{EDD}^{13}$ (Jacobs et al. 2009). The zero-point in this compilation was established by Rizzi et al. (2007) based on horizontal branch/RR Lyrae distances to nearby dwarf spheroidals and other minor galaxies in the Local Group. The sample is expanded by the addition of three objects studied by Jang \& Lee $(2015,2017)$.

${ }^{13}$ http://edd.ifa.hawaii.edu
As with the cluster samples, the zero-point calibrators are subject to faint-limit luminosity cuts. The cut at the optical bands is set at the $i$ band such that galaxies fainter than $M_{i}=-17$ are dropped. The infrared cut is the roughly equivalent $M_{W 1}=-16.1$. There are four galaxies brighter than these limits that deviate by more than $3 \sigma$ from the TFR and are considered anomalous cases and rejected: PGC 5896 (NGC 625), PGC 44536 (NGC 4861), PGC 48334 (NGC 5253), and PGC 68535 (NGC 7250). The optical bands are left with 39 calibrators: 19 CPLR and 29 TRGB, with nine in common. There are 64 calibrators in the infrared: 29 CPLR and 52 TRGB, with 17 in common. There are fewer optical calibrators because of the restricted domain of the SDSS photometry. The WISE photometry is available across the full sky.

Zero-point calibrations are shown for the representative $i$ and $W 1$ bands in Figure 6. The linear fits obey the formulation

$$
\mathcal{M}_{\lambda}^{*}=\operatorname{Slope}\left(\log W_{m x}^{i}-2.5\right)+\mathrm{ZP},
$$

where ZP is the absolute luminosity of a galaxy at $\log W_{m x}^{i}=2.5$. We adopt the convention that the ITFR predictions of absolute magnitudes are given in script. The superscript asterisks indicate that magnitudes are corrected for the effects explained following Equation (2). In Figure 6, the TRGB and CPLR calibrators are shown separately in the middle and bottom panels, respectively, and the combined samples are shown in the top panels, with straight averaging between TRGB and CPLR when both are available.

Slopes are set by the values determined from the cluster templates, while zero-points are established by least-squares regression with the zero-point as the only free parameter. In Figure 6, the maroon dotted lines indicate the location of the zero-point on the absolute magnitude axis. In these examples,

$$
\begin{aligned}
\mathcal{M}_{i}^{*}= & -(20.80 \pm 0.10) \\
& -(8.32 \pm 0.13)\left(\log W_{m x}^{i}-2.5\right), \\
\mathcal{M}_{W 1}^{*}= & -(20.36 \pm 0.07) \\
& -(9.47 \pm 0.14)\left(\log W_{m x}^{i}-2.5\right) .
\end{aligned}
$$

The zero-points for all wave bands are recorded in column (8) of Table 2. Column (7) of this table gives the number of zeropoint calibrators used at each band, and column (9) gives the rms scatter of calibrators about the fitted lines along the absolute luminosity axis.

The distance modulus, $\mu_{\lambda}$, of a given galaxy with the apparent magnitude $m_{\lambda}^{*}$ is given as

$$
\mu_{\lambda}=m_{\lambda}^{*}-\mathcal{M}_{\lambda}^{*}
$$



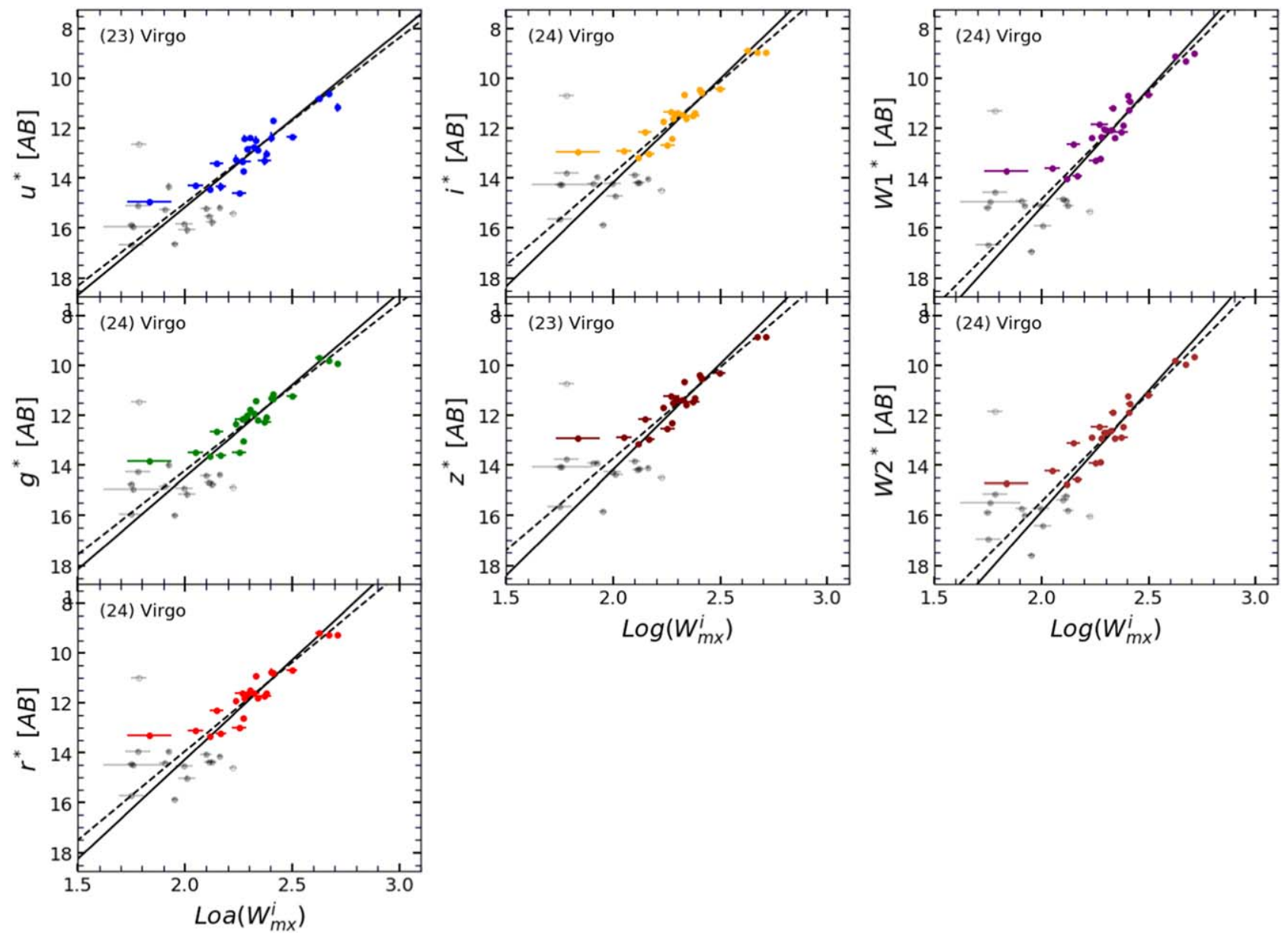

Figure 2. The ITFR fit for the Virgo cluster galaxies at different optical and infrared bands. The dashed line illustrates the fitted ITFR using the filled colored points, and the solid line is the fitted ITFR using the universal slope as a fixed parameter. Gray points represent galaxies that either are outliers or their absolute SDSSmagnitudes, after the full calibration, they are fainter than $M_{i}=-17$ mag.

For example, the magnitude offsets between apparent magnitudes at $\log W_{m x}^{i}=2.5$ in Figure 5 and absolute magnitudes at the same line width value in Figure 6 give measurements for the distance modulus of the Virgo cluster. Moduli for the other clusters can be determined from the magnitude offsets derived in the construction of the universal cluster templates, recorded in the labeling of Figure 5 for the cases of the $i$ and $W 1$ passbands.

\subsection{Alternate Zero-point Calibration: Possible Serious Systematic}

Freedman et al. (2019, hereafter F19) recently provided a recalibration of the TRGB zero-point that led them to derive a value for the Hubble constant $H_{0}$ of $69.8 \pm 1.9$, significantly lower than the Riess et al. (2019) value of $74.0 \pm 1.4$ and, anticipating the results of this study, significantly lower than we find with the calibration derived in Section 3.2. Given the varying degrees of tension of these values with the value of $67.4 \pm 0.5$ derived from early universe conditions (Planck Collaboration et al. 2018) and the implications for physics beyond the standard model, we are called upon to justify why we prefer our TRGB calibration to that of F19.

We note two significant differences between the TRGB zeropoint calibration by F19 and our own. First, F19 based their calibration on the LMC at a distance given by the combination of detached eclipsing binaries (Pietrzyński et al. 2019) and mid-infrared CPLR (Scowcroft et al. 2011; Monson et al. 2012). The LMC is too large, with the red giant branch stars too bright to be easily studied with the Hubble Space Telescope. F19 drew on ground-based observations of the OGLE Collaboration (Ulaczyk et al. 2012) with photometry in the $V$ and $I$ bands. Our calibration is tied to horizontal branch and RR Lyrae distances to the Local Group galaxies M33, IC 1613, NGC 185, Sculptor, and Fornax (Rizzi et al. 2007). The stellar photometry isolating the TRGB was with our standard filters with the Hubble Space Telescope using both the WFPC2 and ACS detectors.

Second, F19 assumed that the TRGB has a constant absolute magnitude of $M_{I}=-4.05$ for all manner of galaxies. ${ }^{14}$ The Rizzi et al. (2007) calibration that we assume incorporates a weak color dependency, in response primarily to metallicity variations and secondarily to age differences. The LMC has a relatively low metal abundance, and consequently, the TRGB is expected in our formulation to be somewhat brighter than is the case with most of the galaxies in the TFR zero-point calibration.

\footnotetext{
${ }^{14}$ Yuan et al. (2019) argued that the F19 TRGB absolute magnitude should be $M_{I}=-3.97$ and the F19 calibration of $H_{0}$ should be increased $3.7 \%$ to $72.4 \mathrm{~km} \mathrm{~s}^{-1} \mathrm{Mpc}^{-1}$, primarily because, they contended, reddening is lower in the direction of the LMC fields than accepted by F19.
} 


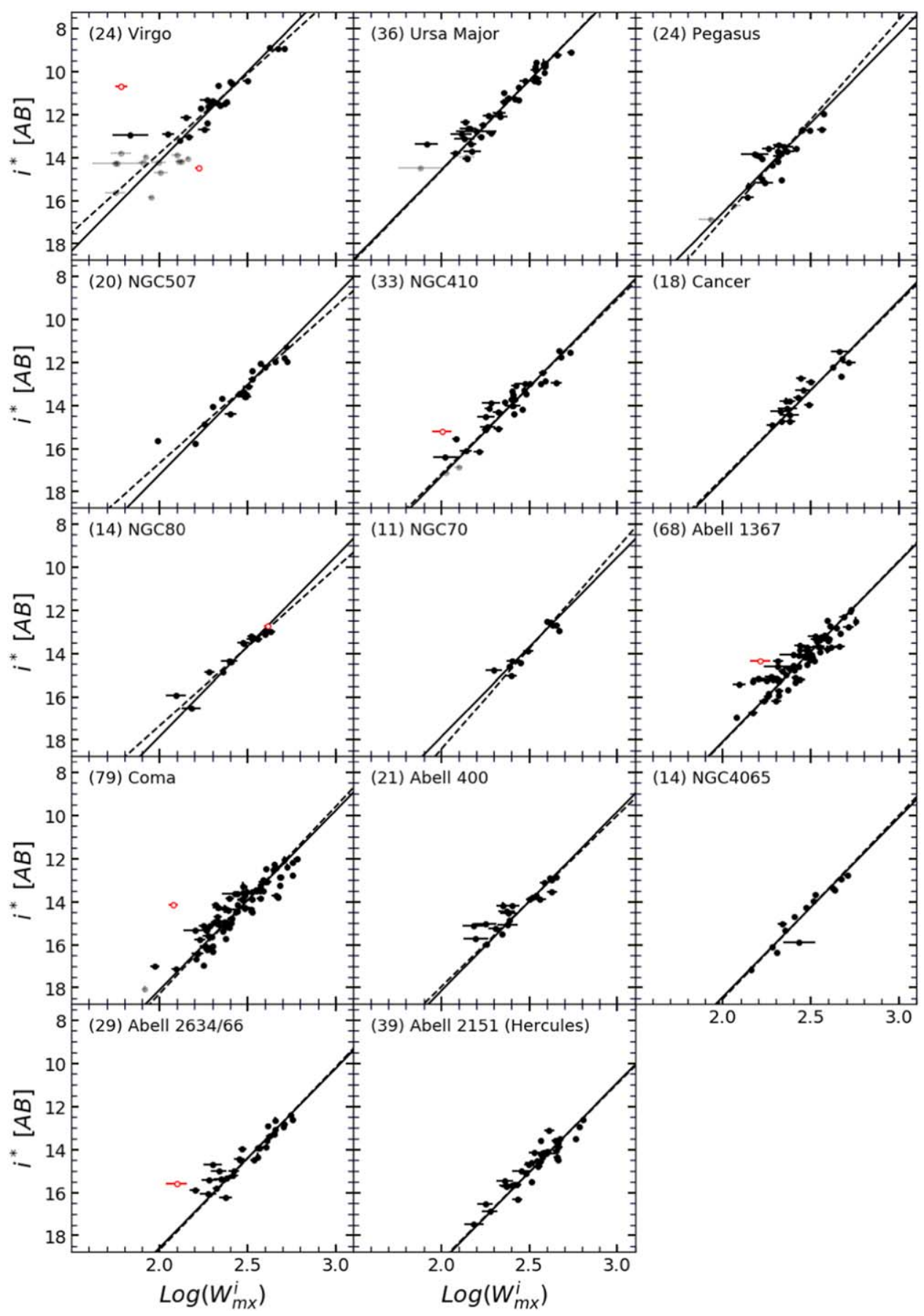

Figure 3. The ITFR fits for 14 clusters with available SDSS $i$-band photometry. In each panel, the dashed line is the fit pertaining to the individual cluster, and the solid line is the best universal ITFR slope fit to the cluster. Red points identify excluded outliers. Gray points are galaxies fainter than the magnitude limit of $M_{i}=-17 \mathrm{mag}$. The number of contributing galaxies in the fitting process is displayed before the cluster name in the top left corner of each panel.

These alternate procedures result in differing values for the Hubble constant, so which better approximates reality? F19 compared their TRGB and Riess et al. (2019) CPLR distance moduli and, for $10 \mathrm{SN}$ Ia systems relevant to their study, they found $\left\langle\mu_{\mathrm{TRGB}}-\mu_{\mathrm{CPLR}}\right\rangle=+0.06 \pm 0.17$ with a $1 \sigma$ uncertainty of \pm 0.05 , evidence for good agreement. We made a similar comparison of our TRGB and Riess et al. (2019) CPLR moduli with 25 galaxies and got the disturbing difference $\left\langle\mu_{\mathrm{TRGB}}-\mu_{\mathrm{CPLR}}\right\rangle=0.13 \pm 0.10$ and a $1 \sigma$ uncertainty of \pm 0.02 .
It could be argued that we should abandon our separate TRGB and CPLR calibrations. Instead, we should force agreement averaged over instances where both measurements are available. We could choose one or the other of the TRGB or CPLR as a baseline or take some average of the two. However, any such approach fails. Consider the panels of Figure 6. Retaining the separation of TRGB and CPLR calibrations, we can reach compatible inferred $H_{0}$ values for the more distant clusters in our study, as will be described culminating in Section 7. However, if one or the other of the TRGB or CPLR 
Table 3

The Parameters of the Fitted ITFRs for Individual Clusters in the $i$ Band, as Visualized in Figure 3

\begin{tabular}{|c|c|c|c|c|c|c|}
\hline Cluster & Code & Ngal & Slope & $\mathrm{ZP}$ & $\mathrm{ZP}_{0}$ & $\mathrm{rms}$ \\
\hline Virgo & $\mathrm{V}$ & 24 & $-7.36 \pm 0.44$ & $10.13 \pm 0.09$ & $10.03 \pm 0.09$ & 0.69 \\
\hline Ursa Major & U Ma & 36 & $-8.39 \pm 0.46$ & $10.43 \pm 0.08$ & $10.43 \pm 0.07$ & 0.53 \\
\hline Pegasus & $\mathrm{Pe}$ & 24 & $-9.27 \pm 1.15$ & $12.24 \pm 0.23$ & $12.40 \pm 0.11$ & 0.59 \\
\hline NGC 507 & N5 & 20 & $-7.27 \pm 0.57$ & $13.06 \pm 0.10$ & $13.06 \pm 0.12$ & 0.54 \\
\hline NGC 410 & N41 & 33 & $-8.10 \pm 0.58$ & $13.15 \pm 0.08$ & $13.14 \pm 0.08$ & 0.52 \\
\hline Cancer & $\mathrm{Ca}$ & 18 & $-8.15 \pm 0.82$ & $13.29 \pm 0.11$ & $13.29 \pm 0.11$ & 0.48 \\
\hline NGC 80 & N8 & 14 & $-7.29 \pm 0.54$ & $13.71 \pm 0.06$ & $13.69 \pm 0.07$ & 0.41 \\
\hline NGC 70 & N7 & 11 & $-9.32 \pm 1.26$ & $13.78 \pm 0.15$ & $13.71 \pm 0.10$ & 0.35 \\
\hline A1367 & A1 & 68 & $-8.40 \pm 0.44$ & $13.91 \pm 0.06$ & $13.91 \pm 0.06$ & 0.56 \\
\hline Coma & $\mathrm{Co}$ & 79 & $-8.68 \pm 0.40$ & $13.94 \pm 0.06$ & $13.95 \pm 0.06$ & 0.56 \\
\hline A400 & A4 & 21 & $-7.95 \pm 0.48$ & $13.99 \pm 0.06$ & $14.00 \pm 0.06$ & 0.58 \\
\hline NGC 4065 & N40 & 14 & $-8.51 \pm 0.50$ & $14.25 \pm 0.09$ & $14.26 \pm 0.08$ & 0.40 \\
\hline A2634/66 & $\mathrm{A} 2$ & 29 & $-8.48 \pm 0.64$ & $14.43 \pm 0.09$ & $14.42 \pm 0.06$ & 0.48 \\
\hline Hercules & $\mathrm{He}$ & 39 & $-8.20 \pm 0.49$ & $15.06 \pm 0.07$ & $15.07 \pm 0.06$ & 0.42 \\
\hline
\end{tabular}

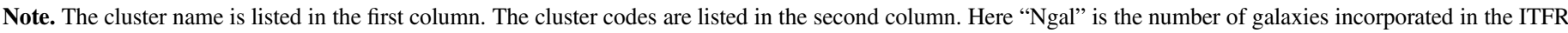

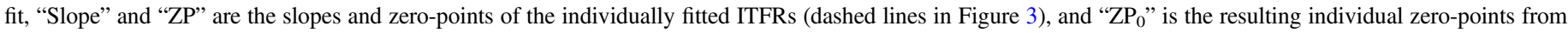

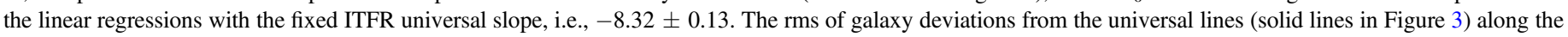
magnitude axis is tabulated in the last column.

scales is changed to force agreement, on average, between individual targets, then a difference at the level of $7 \%$ is created if the zero-point calibration is based solely on TRGB or CPLR samples.

We review this unsatisfactory situation. If the F19 calibration of the TRGB is accepted, then there is statistical agreement between the TRGB measurements and the CPLR moduli of Riess et al. (2019), but there is a disconnect between the TRGB and CPLR calibrations of the TFR that leads to inconsistent determinations of $H_{0}$. If, instead, the TRGB calibration of Rizzi et al. (2007) and the Riess et al. (2019) calibration of the CPLR are accepted, then the TRGB and CPLR calibrations of the TFR are consistent in terms of values of $H_{0}$ found using our cluster sample.

It is to be appreciated that the primary goal of the Cosmicflows program is to measure the peculiar velocities of galaxies, not $H_{0}$. With distances $d_{i}$, we obtain peculiar velocities $V_{\mathrm{pec}, i} \sim c z_{i}-H_{0} d_{i}$, where $H_{0} \sim\left\langle c z_{i} / d_{i}\right\rangle$. Hence, it is important that we use a value of $H_{0}$ compatible with the distance measurements, not that we use the "correct" $H_{0}$. Important details of the zero-point calibration remain to be resolved. The discussion in this section highlights the possibility of substantial systematics.

\subsection{Zero-point Adjustments}

In principle, distance moduli to a given cluster should be the same across all passbands. However, we have detected a trend in the cluster calibrator samples whereby moduli are slightly reduced at blueward bands. We trace this effect to a slight color offset between the ensemble cluster template sample and the sample with CPLR or TRGB distances that gives the zero-point calibration.

The problem is illustrated in Figure 7. Colors with respect to the infrared band $W 1$ are shown on the abscissa in each panel, with the cluster sample plotted as gray points and the zero-point sample in green. The ordinate positions of the points are given by absolute magnitudes in the $W 1$ band following the calibration described in the previous subsection. The solid black lines in these plots are fits to the cluster template sample obeying the formulation $\lambda-W 1=m M_{W 1}+b$. The intercept $b$ is the average $\lambda-W 1$ color of galaxies with $M_{W 1}=0$. The least-squares minimization is carried out in the color terms to counter Malmquist-type bias.

The green dashed lines in these panels are the fits solely to the zero-point calibrators, preserving the black solid line slopes but giving freedom to the scaling parameter $b$. Offsets between the black and green lines are given by $C_{\mathrm{zp}}=b_{\text {clusters }}-b_{\mathrm{zp}}$. In every case, between an optical bandpass and the infrared bandpass $W 1$, the green dashed lines are offset redward of the black solid lines. Never are the offsets statistically significant. However, making an adjustment for the offsets statistically eliminates the differences in distance moduli measurements as a function of passband. We make the adjustment

$$
\overline{\mathrm{ZP}}=\mathrm{ZP}+C_{\mathrm{zp}},
$$

which gives consistency with moduli determined in the $W 1$ band. There is no assurance that reporting to the $W 1$ band is correct. We take the amplitude of shifts at the level of $3 \%$ in distances as a quantitative estimator of systematic uncertainty.

The final linear calibrations are given in Figure 8 for the five optical and two infrared bands. The points in colors represent galaxies from the cluster template brighter than our magnitude thresholds and not rejected as an outlier. The CPLR and TRGB zero-point galaxies are represented by black points and establish the absolute magnitude scales. Information in each panel includes the number of contributing cluster template and zero-point galaxies, the slopes derived from the cluster template, the zero-points derived directly from the galaxies with CPLR or TRGB distances, and the adjustment parameters, $C_{\text {zp }}$, required to null the color offsets demonstrated in Figure 7. Neither of the infrared band correlations receives an adjustment.

To avoid confusion, the nomenclature for the ITFR with adjusted zero-points is

$$
\overline{\mathcal{M}_{\lambda}^{*}}=\text { Slope }\left(\log W_{m x}^{i}-2.5\right)+\overline{\mathrm{ZP}} .
$$




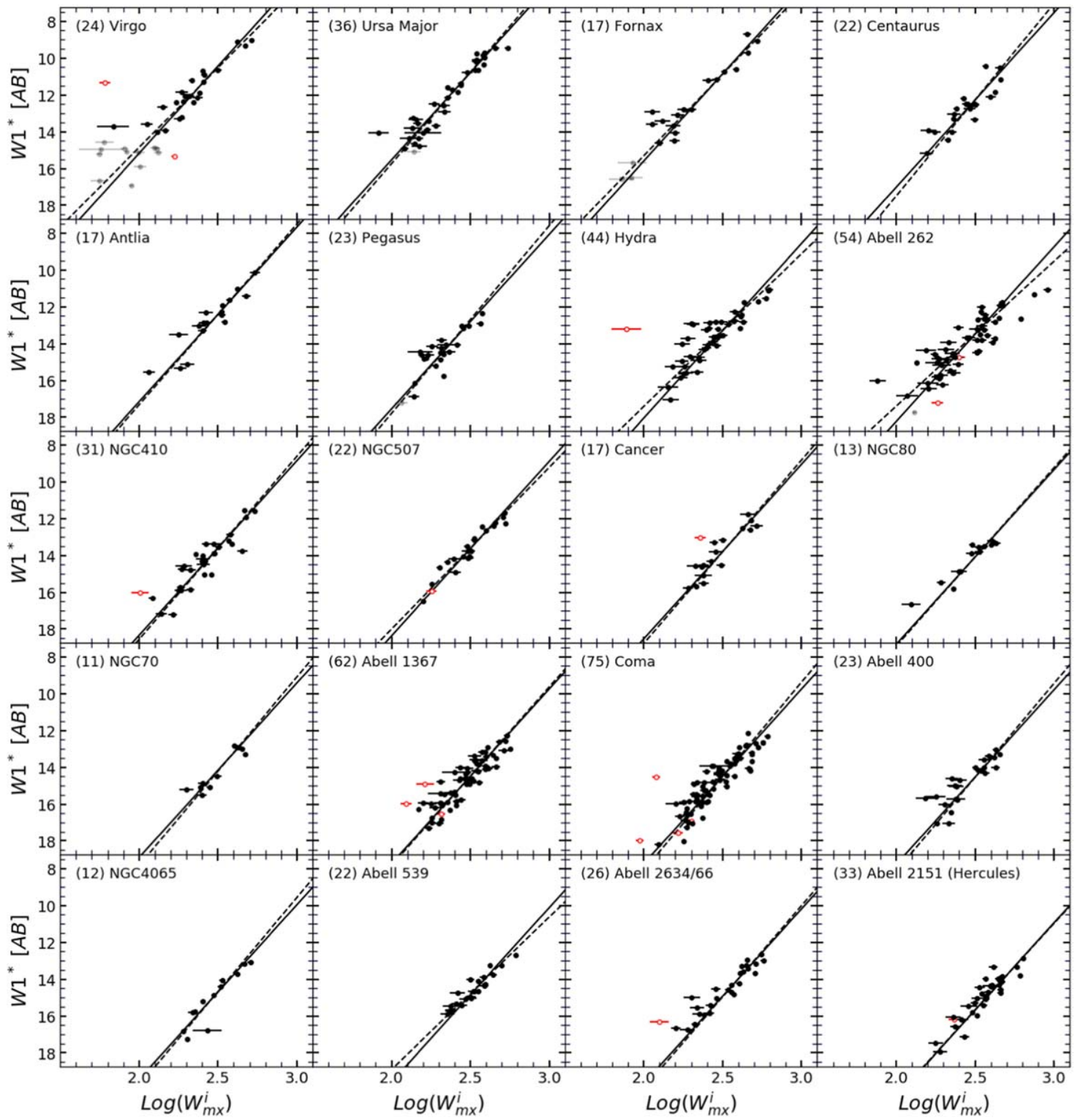

Figure 4. Same as Figure 3 but for 20 clusters with available WISE W1-band photometry. The faint-end magnitude cutoff is at $M_{W 1}=-16.1$ mag.

\subsection{Scatters about the ITFR}

We use the rms scatter of data points about the fitted ITFRs as a measurement of the statistical uncertainty of individual distance estimates. Absolute magnitude residuals from the ITFR are expressed as

$$
\Delta M_{\lambda}^{i}=M_{\lambda}^{i}-\mathcal{M}_{\lambda}^{*},
$$

where $M_{\lambda}^{i}$ is the absolute magnitude of a target galaxy and $\mathcal{M}_{\lambda}^{*}$ is the estimated absolute magnitude at the target's line width from the fitted linear relation. The rms scatter of the ensemble of galaxies in Figure 8 (colored cluster template sample) and zero-point calibrators (black points) are listed in columns (6) and (9) of Table 2, respectively. Residuals $\Delta M_{\lambda}^{i}$ follow an approximately Gaussian distribution; therefore, the rms value can be taken as the $1 \sigma$ error in estimates of target distances.

The averaged $u$-band scatter of 0.60 mag is greater than at longer optical wavelengths. The $u$ band is sensitive to young stellar populations and dust obscuration. Moreover, the quality of photometry is worst at the $u$ band, where the uncertainties of measured magnitudes are $\sim 0.10 \mathrm{mag}$, about double that at other SDSS bands. At the $g, r, i$, and $z$ bands, the averaged rms scatter is relatively the same and equal to $\sim 0.50$ mag. Using 267 cluster galaxies, Tully \& Courtois (2012) reported an rms scatter of $0.41 \mathrm{mag}$ for the I-band (Vega) calibration of the ITFR. With an additional 34 galaxies, Neill et al. (2014) found a slightly larger scatter of 0.46 mag at the same wave band. Our averaged rms scatter about the $i$-band relation, based on 430 galaxies, is 0.49 mag. Scatter tends to increase toward the fainter end of the TFR, a domain more completely probed by our more extensive samples. The increased scatter toward fainter magnitudes is demonstrated in Figure 9. Scatter increases from $\sim 0.40$ for the most luminous galaxies to $\sim 0.58$ for the faintest galaxies. It can be noted that the rms scatter for individual clusters recorded in Tables 3 and 4 tends to decrease for the more distant clusters. Progressively with increasing distance, only the bright end of the TFR is probed.

Based on 584 galaxies in our sample with infrared photometry, we find that the dispersion about the $W 1$ and $W 2$ calibrations is 0.58 and $0.62 \mathrm{mag}$, respectively, in agreement 

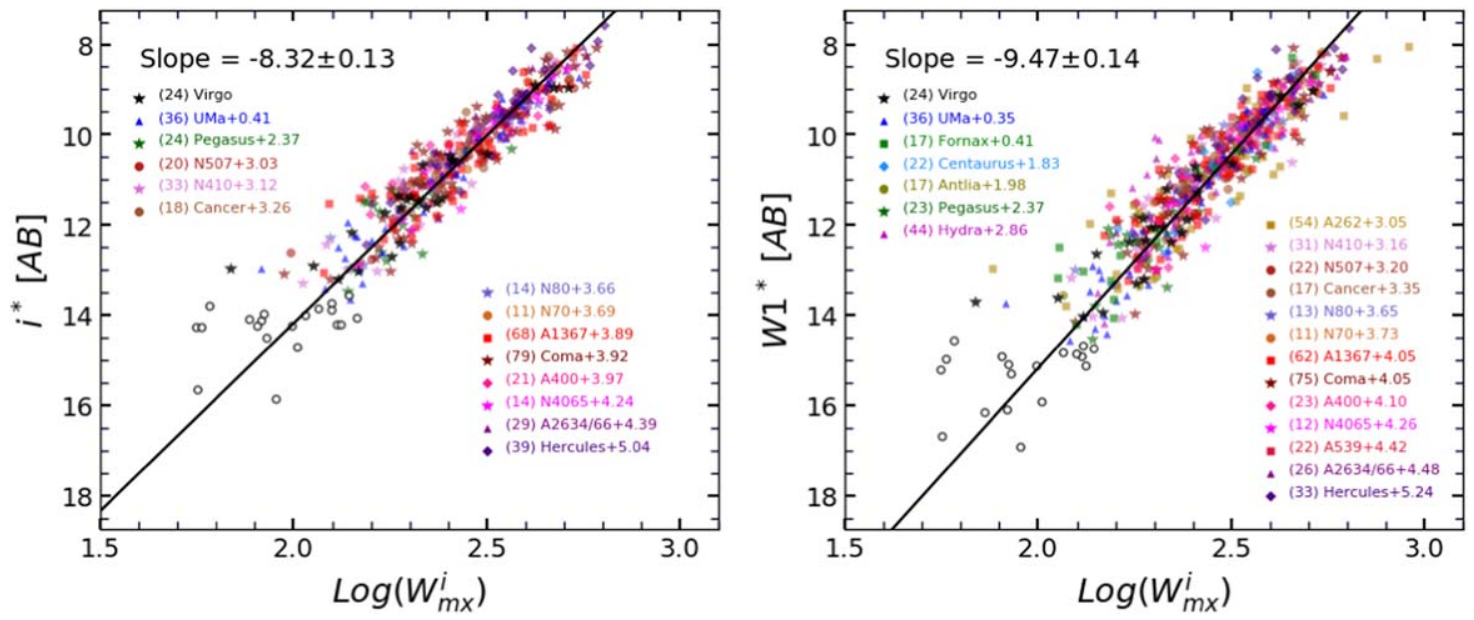

Figure 5. Universal template constructed from all clusters to find the slope of the ITFR. All clusters are shifted forward to the distance of the Virgo cluster by adjusting the apparent magnitude of their constituent galaxies. Galaxies in each cluster are displayed by unique symbol/color. Labels start with the number of contributed galaxies in each cluster, followed by the cluster name and the magnitude offset relative to Virgo. Open circles are galaxies fainter than the faint-end magnitude cutoff, i.e., $-17 \mathrm{mag}$ at the $i$ band and $-16.1 \mathrm{mag}$ at the $W 1$ band.

Table 4

Same as Table 3 but for the Parameters of the Fitted ITFRs for Individual Clusters in the $W 1$ Band, as Visualized in Figure 4

\begin{tabular}{|c|c|c|c|c|c|c|}
\hline Cluster & Code & Ngal & Slope & $\mathrm{ZP}$ & $\mathrm{ZP}_{0}$ & $\mathrm{rms}$ \\
\hline Virgo & V & 24 & $-8.64 \pm 0.53$ & $10.53 \pm 0.11$ & $10.44 \pm 0.10$ & 0.82 \\
\hline Ursa Major & U Ma & 36 & $-9.94 \pm 0.49$ & $10.77 \pm 0.08$ & $10.80 \pm 0.08$ & 0.59 \\
\hline Fornax & F & 17 & $-8.86 \pm 0.61$ & $10.88 \pm 0.13$ & $10.86 \pm 0.14$ & 0.78 \\
\hline Centaurus & $\mathrm{Ce}$ & 22 & $-10.44 \pm 1.27$ & $12.27 \pm 0.15$ & $12.28 \pm 0.13$ & 0.59 \\
\hline Antlia & An & 17 & $-9.80 \pm 1.05$ & $12.43 \pm 0.12$ & $12.42 \pm 0.11$ & 0.61 \\
\hline Pegasus & $\mathrm{Pe}$ & 23 & $-10.19 \pm 1.21$ & $12.70 \pm 0.24$ & $12.81 \pm 0.12$ & 0.66 \\
\hline Hydra & Hy & 44 & $-8.19 \pm 0.55$ & $13.25 \pm 0.09$ & $13.29 \pm 0.10$ & 0.89 \\
\hline A262 & A26 & 54 & $-7.86 \pm 0.63$ & $13.47 \pm 0.11$ & $13.49 \pm 0.13$ & 0.98 \\
\hline NGC 410 & N41 & 31 & $-9.93 \pm 0.83$ & $13.60 \pm 0.11$ & $13.61 \pm 0.10$ & 0.64 \\
\hline NGC 507 & N5 & 22 & $-8.78 \pm 0.52$ & $13.61 \pm 0.08$ & $13.64 \pm 0.08$ & 0.38 \\
\hline Cancer & $\mathrm{Ca}$ & 17 & $-9.95 \pm 0.84$ & $13.80 \pm 0.12$ & $13.79 \pm 0.11$ & 0.51 \\
\hline NGC 80 & N8 & 13 & $-9.68 \pm 1.23$ & $14.09 \pm 0.13$ & $14.09 \pm 0.12$ & 0.53 \\
\hline NGC 70 & N7 & 11 & $-10.23 \pm 1.40$ & $14.22 \pm 0.16$ & $14.17 \pm 0.12$ & 0.42 \\
\hline A1367 & A1 & 62 & $-9.70 \pm 0.52$ & $14.49 \pm 0.06$ & $14.49 \pm 0.06$ & 0.59 \\
\hline Coma & Co & 75 & $-10.21 \pm 0.48$ & $14.49 \pm 0.07$ & $14.50 \pm 0.06$ & 0.61 \\
\hline A400 & A4 & 23 & $-10.19 \pm 0.76$ & $14.55 \pm 0.09$ & $14.54 \pm 0.08$ & 0.69 \\
\hline NGC 4065 & $\mathrm{~N} 40$ & 12 & $-10.21 \pm 0.87$ & $14.68 \pm 0.12$ & $14.71 \pm 0.11$ & 0.53 \\
\hline A539 & A5 & 22 & $-8.28 \pm 0.66$ & $14.77 \pm 0.08$ & $14.86 \pm 0.08$ & 0.39 \\
\hline A2634/66 & $\mathrm{A} 2$ & 26 & $-9.91 \pm 0.92$ & $14.97 \pm 0.12$ & $14.93 \pm 0.08$ & 0.57 \\
\hline Hercules & $\mathrm{He}$ & 33 & $-9.38 \pm 0.82$ & $15.68 \pm 0.12$ & $15.68 \pm 0.09$ & 0.52 \\
\hline
\end{tabular}

Note. The universal slope at the $W 1$ band is $9.47 \pm 0.14$.

with previous studies (Sorce et al. 2013; Neill et al. 2014). The latter reported a scatter of 0.54 mag for dispersion about their W1 ITFR fit using 310 galaxies.

Overall, scatter tends to decrease toward the near-infrared wavelengths where with the effects of young stellar population and metallicity are minimized, as anticipated by Aaronson et al. (1979). The subsequent substantial increase in scatter at the WISE bandpasses is associated with a color term that is discussed in Section 4.

\subsection{Cluster Distance Bias}

While the formalism of the ITFR effectively nulls the selection Malmquist bias, another bias remains because of the nonuniform population of the luminosity function of a cluster. Here our concern is restricted to only the gas-rich spiral populations, which, from compilations combining Virgo, Ursa Major, and Fornax samples, can be described by Schechter functions (Schechter 1976) with bright-end exponential cutoffs characterized by $M_{I}^{\star}=-23.0$ and $M_{W 1}^{\star}=-22.0$ and faint-end power-law slopes $\alpha=-1.0$ (Tully \& Courtois 2012; Neill et al. 2014).

The bias arises when the faint limit in the sampling of a cluster approaches the exponential cutoff magnitude. In such instances, there are more fainter galaxies available to scatter upward into the sample than brighter galaxies to scatter downward out of the sample. The upward-scattered systems will tend to lie above an unbiased relation, resulting in a fit to the cluster that is slightly too close. Since the Schechter faintend slope parameter $\alpha=-1.0$ implies equal numbers per magnitude bin at fainter magnitudes, for relatively nearby 

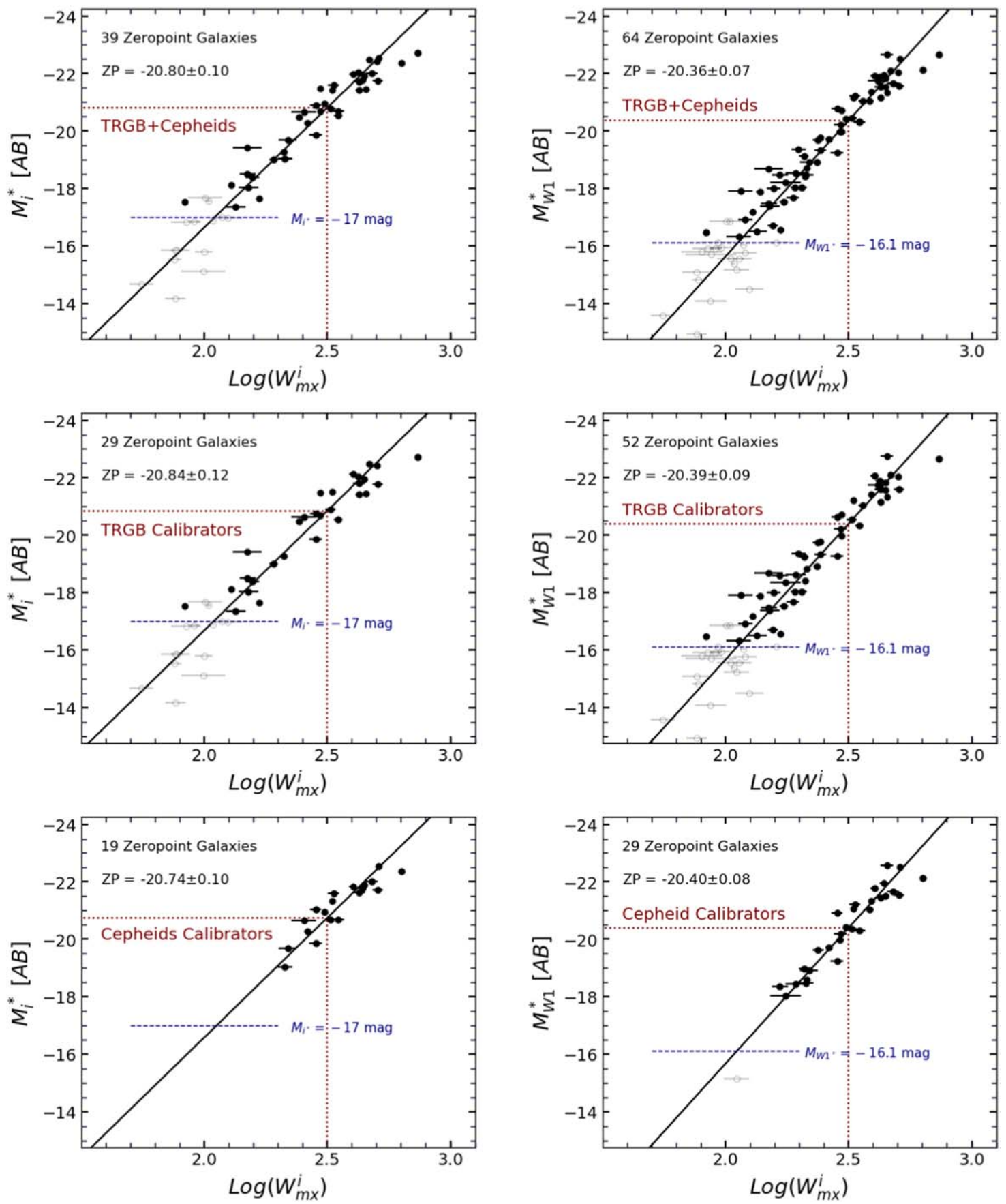

Figure 6. Absolute luminosity of the zero-point calibrators vs. H I line width at the optical $i$ band (left) and infrared $W l$ band (right). The slopes of the solid lines are set by the cluster template. Least-squares minimization of magnitudes sets the zero-points. Blue dashed lines mark the adopted magnitude limits. Faint and excluded zero-point calibrators are displayed in gray. Maroon dotted horizontal/vertical lines mark magnitude zero-points $\operatorname{where} \log \left(W_{m x}^{i}\right)=2.5$.

clusters, there is little bias because there is a roughly equal probability of upward and downward scatter. See Neill et al. (2014) for further elaboration.

We determine the amplitudes of the bias in each passband with simulations. However, before discussing that detail, we address another concern. Simulations can be used to study the joint effects discussed in this section and the next.

\subsection{TFR Curvature}

A close look at Figure 8 reveals that at the bright end of the TFR, almost all galaxies are below the fitted straight lines, implying that the linear TFR might not perfectly capture the general trend of data points. The departure of bright galaxies from the linear TFR is larger at longer wavelengths. Here we treat the effect of curvature as an additional bias that is addressed together with the effect of magnitude cutoff bias.

Curvature of the near-IR TFR has been considered in previous studies using $H$-band luminosities (Aaronson et al. 1986, and references therein). Sakai et al. (2000) used quadratic functions for the $B V R I H_{-0.5}$ bands and showed that the curvature term increases with wavelength, which is consistent with what we observe in Figure 8. Noordermeer \& Verheijen (2007) described the effect with linear fits with a break between two slopes, noting systems with declining rotation curves at 

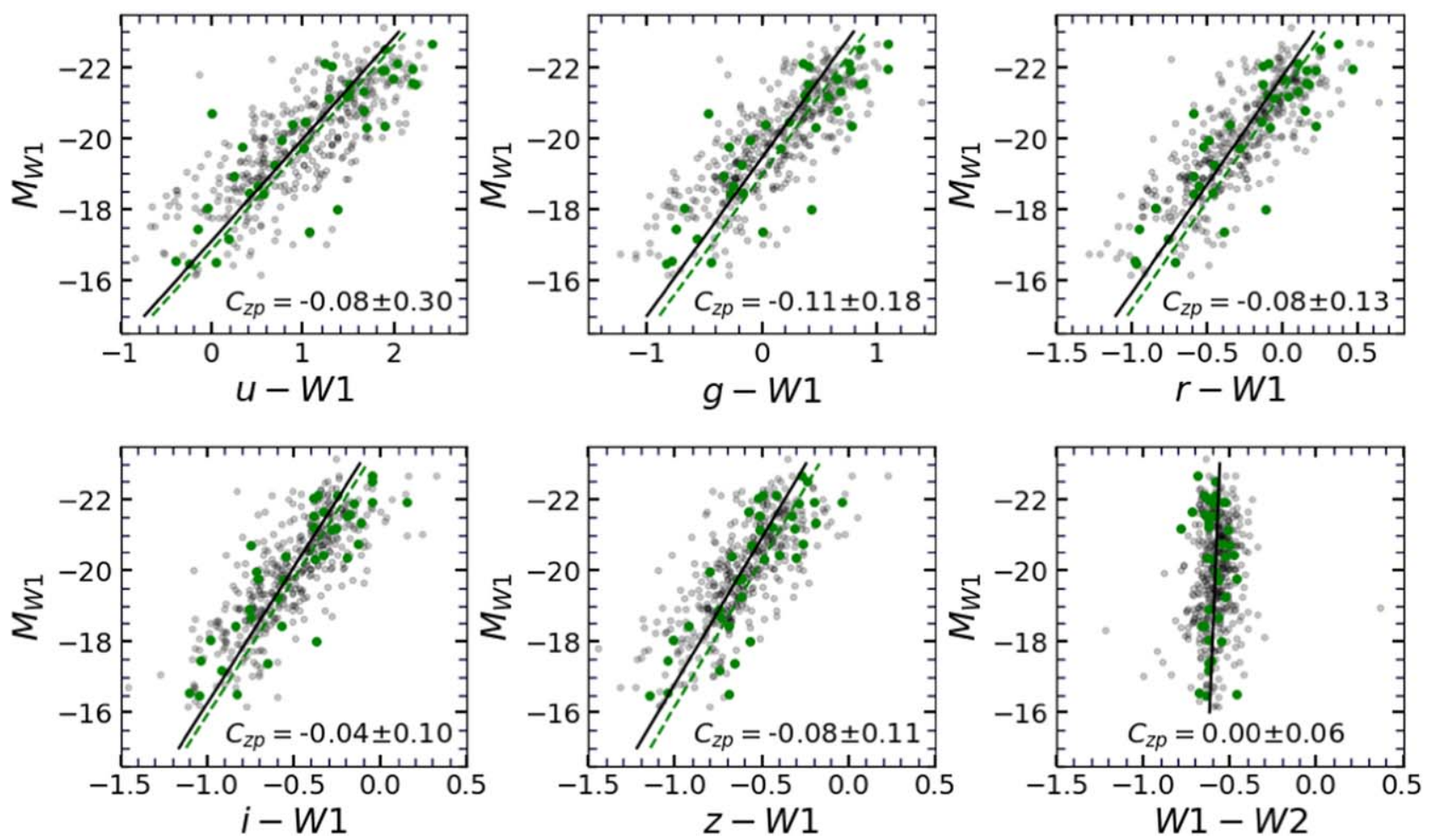

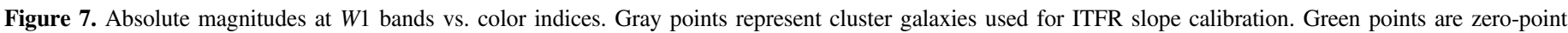

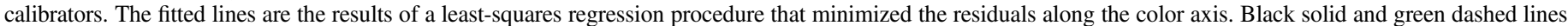

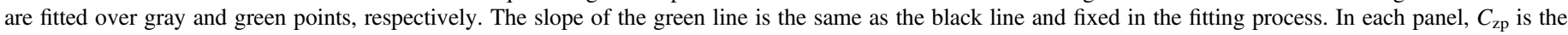
difference in the zero-point of the fitted lines along the horizontal axis.

large radii dominate the flatter portion at the bright end and that formulating a "baryonic" relation (McGaugh 2005) helps to minimize the change in slopes. Neill et al. (2014) accounted for curvature with the empirical strategy of employing quadratic functions for the TFR without adjustments of magnitudes or line widths.

In this study, we accept the fitted ITFRs everywhere except for the bright end, where we model the deviations of data points from the linear relation using quadratic functions. Our attempt to fit an inverted quadratic relation failed due to the dramatic increase of scatter about the resulting TFR. The same issue was also reported by Neill et al. (2014). Here, to fit the quadratic relation, we take the direct fitting approach and let the regression process minimize residuals along the magnitude axis. To keep the Malmquist bias at a minimum when fitting the quadratic curves, we still use the same template we constructed in the slope calibration process (see Section 3.1). The uncertainties of the magnitudes are much smaller than that of line widths; thus, we project the line width error bars onto the magnitude axis based on the slope of the linear TFR.

In our analysis, we examined different locations for the break point, where there is the transition from linear to quadratic forms. At optical bands, taking the break point as a free parameter of the fit results in unrealistic fitted curves, with harsh deviations from the linear relation beginning at $\log$ $\left(W_{m x}^{i}\right) \sim 2.6$ and a turnover after reaching to a maximum within the range of the data. Rather, at optical bands, we empirically explored break points to obtain a result that curves gently from the linear relations while leaving enough data points for the fitting process. We adopted $\log \left(W_{m x}^{i}\right)=2.5$ for the onset of deviation. At infrared bands, by contrast, the break point is reasonably found from minimization in the fitting process, giving $\log \left(W_{m x}^{i}\right)=2.4$. The following quadratic relation formulates the deviation from the linear TFR after the break point,

$$
\Delta M_{\lambda}=A_{2} X^{2}+A_{1} X+A_{0}
$$

where $X=\log \left(W_{m x}^{i}\right)-2.5$. Our fitting procedure requires that the quadratic curve and the linear TFR share the same slope at the break point, which reduces the number of independent parameters. Table 5 lists $A_{2}$ and other essential parameters in the calculation of $A_{1}$ and $A_{0}$. Figure 10 displays the resulting curved relations that apply brightward of the break points, with the previously determined linear ITFRs faintward of the break points. The rms scatter of the magnitude offsets from the curved relations is reported in the last column of Table 5. Scatter is not substantially reduced compared to the linear ITFRs because a large fraction of the luminosity-line width correlation is still modeled linearly, the deviation of the curved region from linear is modest, and there are not that many galaxies affected by the curvature at the bright end.

\subsection{Simulations}

We follow a similar bias analysis as explained in Tully \& Courtois (2012), Sorce et al. (2013), and Neill et al. (2014), with one important difference. Those earlier studies only considered the bias discussed in Section 3.6 while now we also consider the bias due to curvature, the topic of Section 3.7. These two biases act in opposite senses, and we study their effects jointly with simulations. 


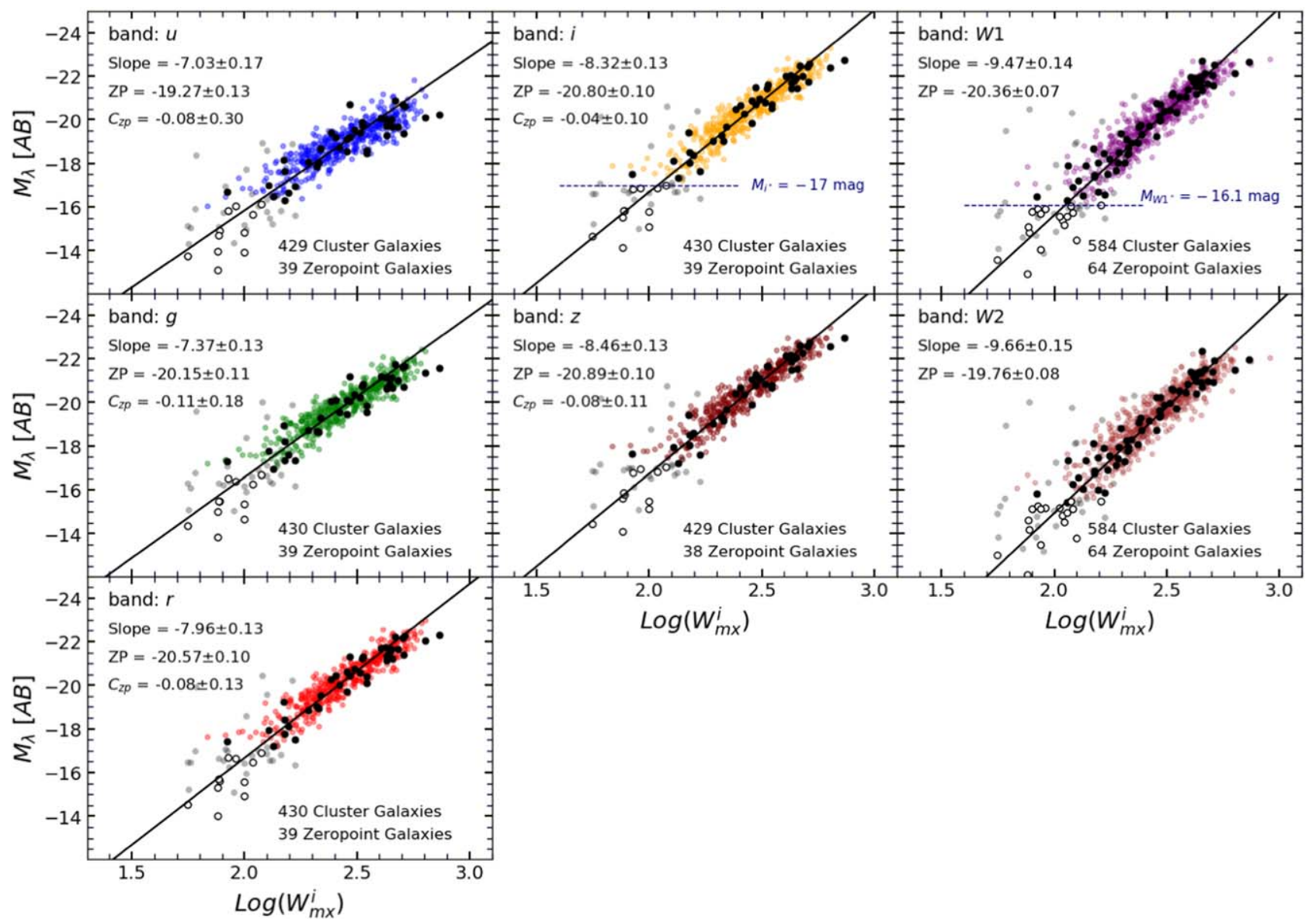

Figure 8. Absolute luminosity vs. H I line width for different wave bands. Solid straight lines illustrate the fitted ITFRs with no bandpass adjustments. Colored points show cluster galaxies that are used for slope calibration. The magnitude limit $M_{i}=-17$ mag is imposed in the fitting process, illustrated by the blue dashed line in the $i$-band panel. The blue dashed line in the W1-band panel shows the equivalent magnitude limit of -16.1 in the $W 1$ band. Gray points are the faint cluster galaxies or the outliers we excluded from the ensemble in the fitting process. Black filled points are the spirals used for zero-point calibration. Open circles are zero-point calibrators that are fainter than the adopted magnitude limit.

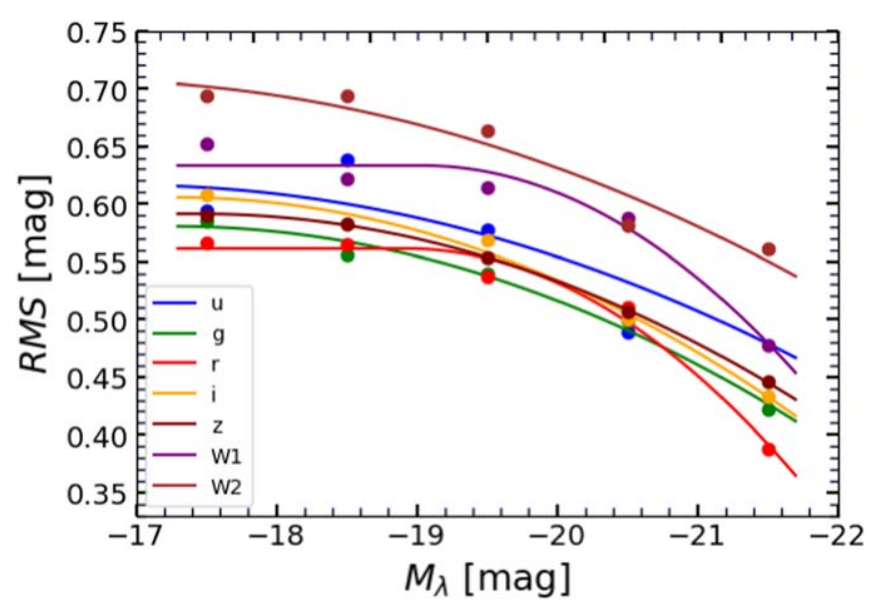

Figure 9. Binned rms scatter of the template cluster galaxies about the linear ITFR at different passbands. Curves are fitted parabolic functions that are flattened after they peak at the faint end.

We approximate the luminosity function of TFR-applicable galaxies with the Schechter function (Schechter 1976). To determine the faint-end slope, $\alpha$, we fit to the combination of Virgo-Ursa Major-Fornax cluster samples. The bright-end cutoff parameter $M^{\star}$ is poorly constrained by only these three clusters, so, to improve statistics, we add clusters by distance
Table 5

Parameters of the Curved TFRs

\begin{tabular}{lccccc}
\hline \hline Band & Slope & ZP & $\begin{array}{c}\text { log } \\
\left(W_{m x}^{i}\right) \\
\text { Break } \\
\text { Point }\end{array}$ & $A_{2}$ & rms \\
& & & & \\
& & & & \\
& & & & \\
\hline$u$ & $-7.03 \pm 0.17$ & $-19.27 \pm 0.13$ & 2.5 & $6.59 \pm 1.10$ & 0.56 \\
$g$ & $-7.37 \pm 0.13$ & $-20.15 \pm 0.11$ & 2.5 & $4.18 \pm 0.90$ & 0.48 \\
$r$ & $-7.96 \pm 0.13$ & $-20.57 \pm 0.10$ & 2.5 & $4.56 \pm 0.89$ & 0.48 \\
$i$ & $-8.32 \pm 0.13$ & $-20.80 \pm 0.10$ & 2.5 & $5.34 \pm 0.91$ & 0.48 \\
$z$ & $-8.46 \pm 0.13$ & $-20.89 \pm 0.10$ & 2.5 & $5.81 \pm 0.91$ & 0.48 \\
$W 1$ & $-9.47 \pm 0.14$ & $-20.36 \pm 0.07$ & 2.4 & $3.81 \pm 0.42$ & 0.56 \\
$W 2$ & $-9.66 \pm 0.15$ & $-19.76 \pm 0.08$ & 2.4 & $4.42 \pm 0.43$ & 0.61 \\
\hline
\end{tabular}

Note. Slope and ZP are parameters of the linear TFR.

groups, with $\alpha$ fixed by the nearest three clusters and magnitude ranges for the determination of $M^{\star}$ increasingly constrained with distance. The fitted Schechter parameters at the different bands are given in Table 6 .

The process of generating synthetic samples is given by the following recipe. (1) At each band, we generate a synthetic sample of galaxies with absolute magnitudes drawn from the Schechter luminosity function. (2) We assign a synthetic line width to each simulated galaxy based on the curved luminosity-line width correlations presented in Section 3.7. 


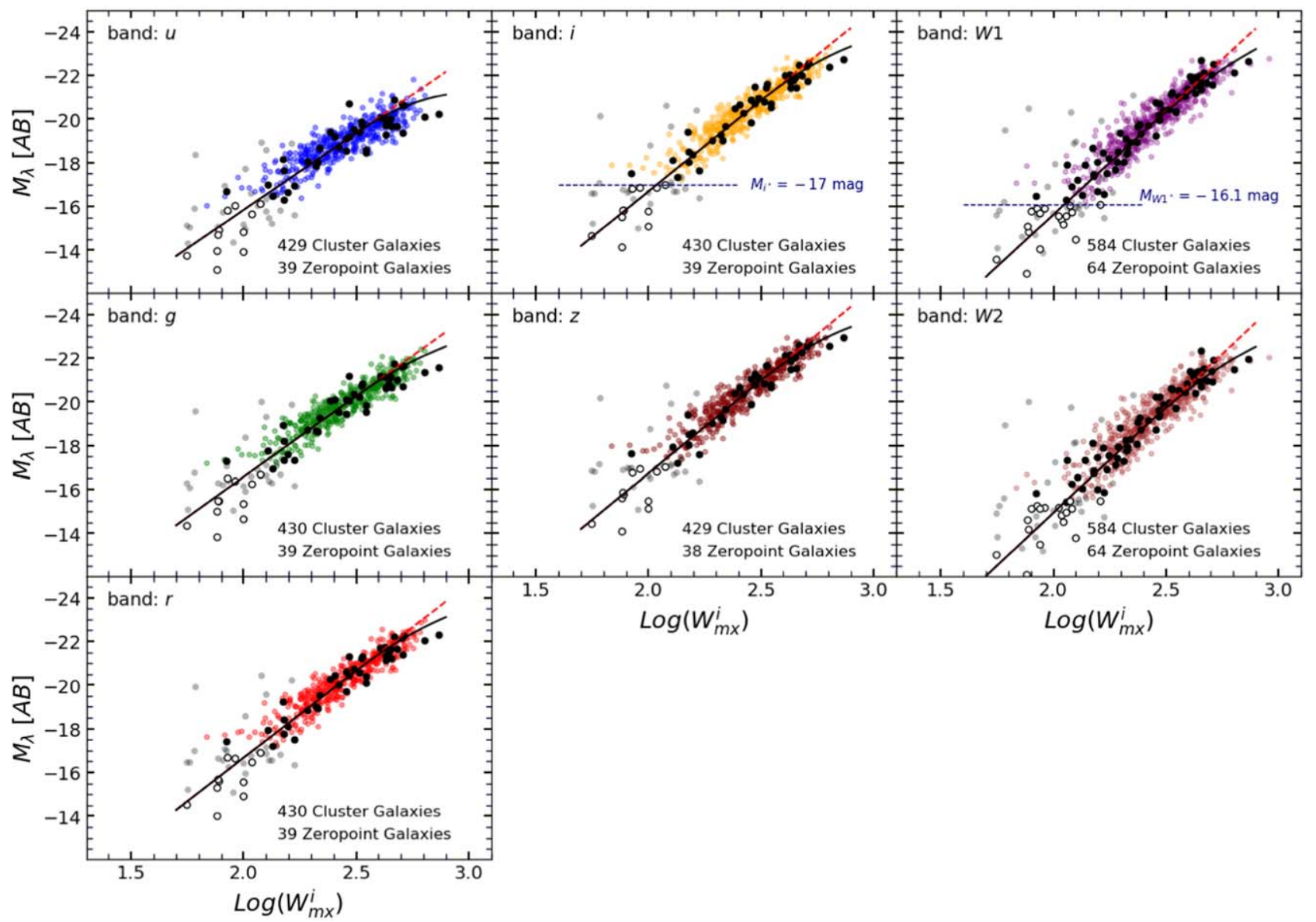

Figure 10. Similar to Figure 8. The linear ITFR is replaced with quadratic functions at the bright ends. At the $u, g, r, i$, and $z$ optical bands, the departure from the linear relation begins at $\log \left(W_{m x}^{i}\right)=2.5$. At the $W 1$ and $W 2$ infrared bands, the break point is at $\log \left(W_{m x}^{i}\right)=2.4$. In each panel, the dashed line displays the continuation of the linear ITFR.

Table 6

Parameters of the Third-degree Polynomial Function, $b=0.01 \times \sum_{n=0}^{3} B_{n} M_{\mathrm{lim}}^{n}$, that Fits the Distance Modulus Bias at Different Wave Bands

\begin{tabular}{|c|c|c|c|c|c|c|c|}
\hline \multirow{2}{*}{ Band } & \multirow{2}{*}{$\begin{array}{l}\text { TFR } \\
\text { Code }\end{array}$} & \multicolumn{4}{|c|}{ Modulus Bias Function } & \multicolumn{2}{|c|}{ LF Parameters } \\
\hline & & $B_{3}$ & $B_{2}$ & $\bar{B}$ & $B_{0}$ & $M^{\star}$ & $\alpha$ \\
\hline$u$ & $\mathrm{TF}_{c}$ & $1.08 \pm 0.19$ & $3.73 \pm 0.95$ & $-0.87 \pm 1.23$ & $0.16 \pm 0.38$ & $-19.9 \pm 0.3$ & $-1.0 \pm 0.3$ \\
\hline$g$ & $\mathrm{TF}_{g}$ & $0.41 \pm 0.03$ & $1.57 \pm 0.21$ & $-0.00 \pm 0.35$ & $0.12 \pm 0.15$ & $-21.3 \pm 0.5$ & $-1.0 \pm 0.2$ \\
\hline$r$ & $\mathrm{TF}_{r}$ & $0.48 \pm 0.04$ & $1.82 \pm 0.24$ & $0.37 \pm 0.43$ & $0.18 \pm 0.19$ & $-21.8 \pm 0.2$ & $-1.0 \pm 0.1$ \\
\hline$i$ & $\mathrm{TF}_{i}$ & $0.37 \pm 0.02$ & $1.26 \pm 0.14$ & $-0.28 \pm 0.25$ & $0.11 \pm 0.12$ & $-22.0 \pm 0.1$ & $-1.0 \pm 0.1$ \\
\hline$z$ & $\mathrm{TF}_{z}$ & $0.34 \pm 0.02$ & $1.14 \pm 0.15$ & $-0.30 \pm 0.26$ & $0.09 \pm 0.11$ & $-22.1 \pm 0.2$ & $-1.0 \pm 0.1$ \\
\hline$W 1$ & $\mathrm{TF}_{W 1}$ & $0.21 \pm 0.01$ & $0.61 \pm 0.05$ & $-0.21 \pm 0.10$ & $0.00 \pm 0.04$ & $-21.9 \pm 0.1$ & $-1.0 \pm 0.1$ \\
\hline$W 2$ & $\mathrm{TF}_{W 2}$ & $0.25 \pm 0.02$ & $0.98 \pm 0.15$ & $0.40 \pm 0.28$ & $0.12 \pm 0.14$ & $-21.3 \pm 0.2$ & $-1.0 \pm 0.1$ \\
\hline
\end{tabular}

Note. The last two columns are the fitted parameters of the luminosity function as described by the Schechter function.

(3) The magnitude of each galaxy is then statistically dispersed assuming a normal distribution with standard deviations taken from the scatter models similar to that presented in Figure 9, applied here to the curved luminosity-line width relations. The left panels of Figure 11 illustrate the ensemble of 1000 simulated galaxies at the $i$ and $W 1$ bands.

To calculate the amplitude of bias, for any given faint-end magnitude limit, $M_{\text {lim }}$, we draw 10,000 galaxies randomly with simulated magnitudes brighter than the cutoff magnitude. In our analysis, the relative distance moduli of clusters are determined through fitting the linear luminosity-line width correlations; therefore, we follow the same recipe to measure the magnitudes of the random ensemble. The line widths of randomly selected galaxies determine the assignments of absolute magnitudes from the model TFR. The average deviation of these measured magnitudes from input magnitudes is the bias, $b$.

The right panels of Figure 11 plot the bias as a function of magnitude limits. For each magnitude cutoff, we repeat the calculations for 100 different random ensembles and plot the averages and $1 \sigma$ standard deviations of the results. Data points are normalized to zero at the magnitude limits $M_{i}=-17$ and $M_{W 1}=-16.1 \mathrm{mag}$, where bias for a complete sample goes to zero. In this plot, black solid curves are third-degree 

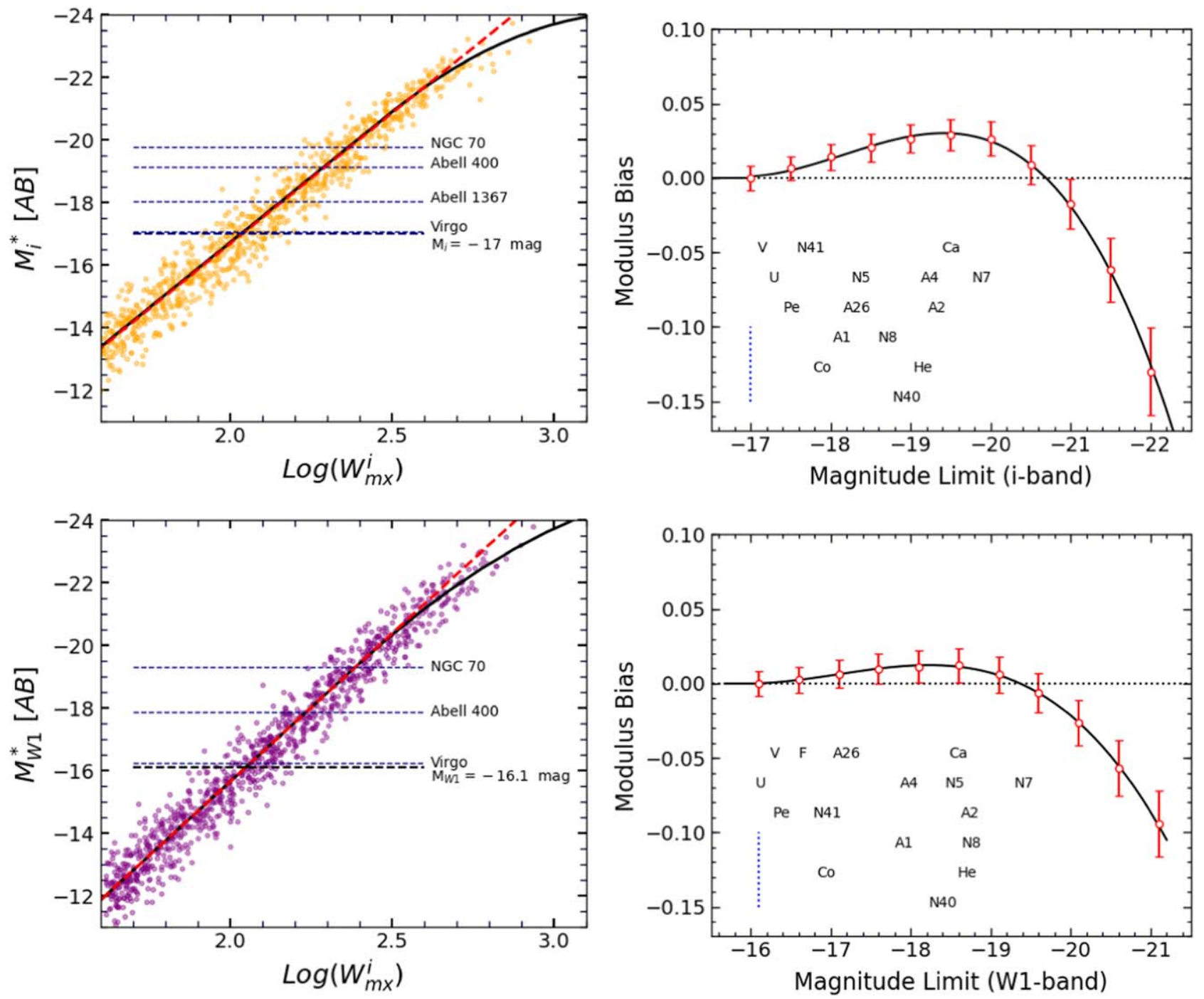

Figure 11. Left: 1000 simulated galaxies along the curved TFR drawing from the appropriate Schechter luminosity function and scattered along the magnitude axis based on the rms scatter model presented in Figure 9. Horizontal dashed lines represent the magnitude limit of our sample at the $i$ and $W 1$ bands. Dashed lines display the faint-end magnitude cutoffs of Virgo, A1367, A400, and NGC 70 clusters as examples. Right: distance bias as a function of the faint-end magnitude limit of simulated TFRs. Cluster codes, placed horizontally, locate the faint-end magnitude coverage of calibrator clusters. Vertical dotted lines are drawn at the location of the magnitude cutoff of our samples at $M_{i}=-17$ and $M_{W 1}=-16.1 \mathrm{mag}$.

polynomial functions that fit the modulus bias, of the form $b=0.01 \times \sum_{n=0}^{3} B_{n} M_{\mathrm{lim}}^{n}$, where $B_{n}$ is the coefficient of the polynomial function. Table 6 lists the coefficients of the fitted polynomials.

It is seen in Figure 11 that the combination of the two biases, those discussed in Sections 3.6 and 3.7 respectively, give results significantly different from those derived by Neill et al. (2014) and earlier because, while the bias due to scatter on the Schechter function is monotonically increasingly positive with distance, the curvature bias is monotonically increasingly negative with distance. With increasing distance, the former bias is initially dominant, but then the latter takes over. Over the range of relevance for our calibrator clusters, the bias remains positive but is subdued, reaching just $b \sim 0.04$ at the $i$ band and $b \sim 0.02$ at the $W 1$ band.

\section{TFR with Additional Parameters}

There have been suggestions to reduce the dispersion in the TFR by including additional parameters such as type, color, or
H I-to-stellar content (Rubin et al. 1985; Giovanelli et al. 1997; Makarov et al. 2018). In this spirit, Masters et al. (2006) fit the TFR at the $I$ band for early- and late-type spirals separately. It has been known that the TFR is steeper at longer wavelengths (Tully et al. 1982). This property must introduce a color term that affects scatter, producing systematic differences between passbands.

Larger spirals rotate faster, as manifested in larger $\mathrm{HI}$ line widths. Larger galaxies are composed of older and redder stellar populations owing to their rapid star formation histories. Consequently, spirals with larger HI line widths (bigger galaxies) are in general redder, as shown in Figure 12, causing the fast-rotating end of the TFR to rise up relative to the slowrotating end when progressing to longer wave bands. This effect is illustrated in Figure 13. The TFR progressively steepens from the optical $u$ band to the infrared $W 2$ band.

Consider two spirals that are different in color but the same in line width. Toward infrared bands, the redder galaxy rises in luminosity relative to the bluer galaxy. This effect induces an intrinsic scatter about the TFR that is a function of wave band and the color distribution of the sample galaxies. 


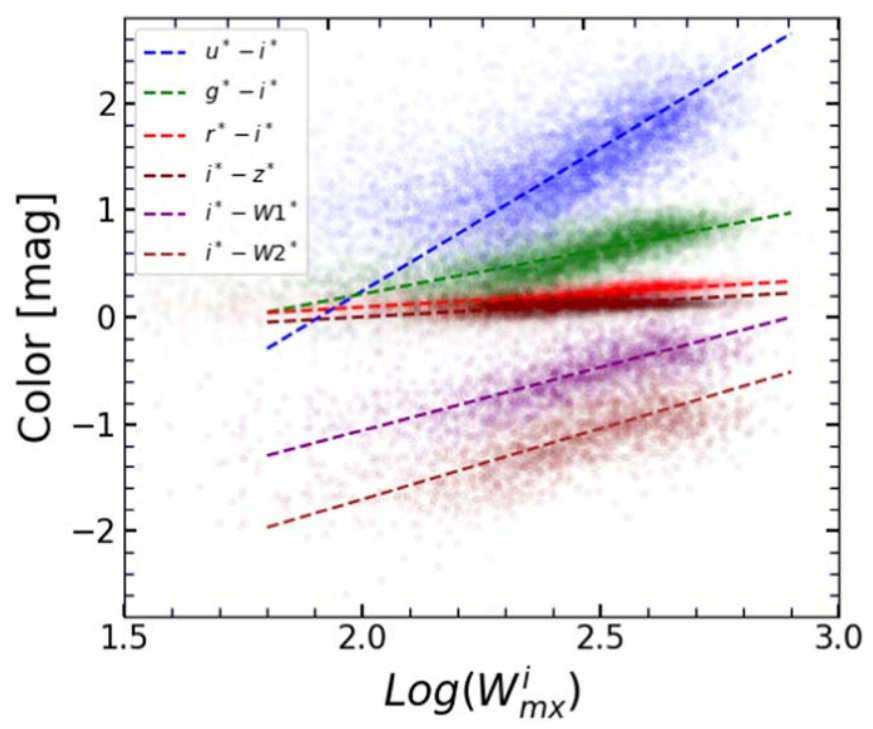

Figure 12. Color terms vs. H I line width. Each point displays a galaxy. Points are colored based on the color indices they represent. Dashed lines fit the data points of corresponding colors using the least-squares regression through minimizing the residuals along the vertical axis. The parameters of the fits are presented in Table 7.

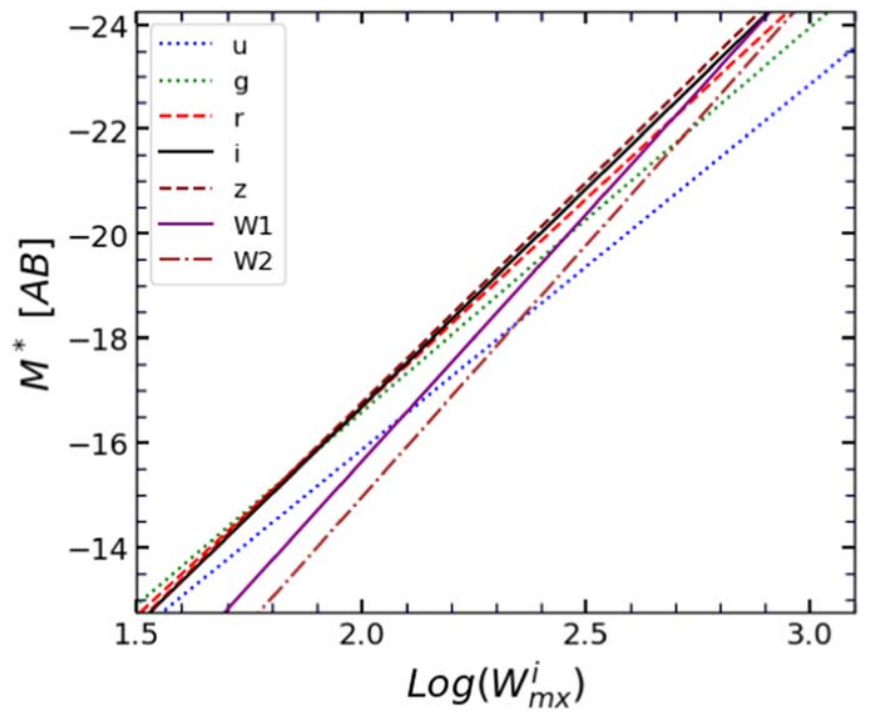

Figure 13. The ITFR fits in different optical and infrared wave bands. The power-law lines are those displayed in Figure 8.

The need for a color term in the infrared was clearly documented by Sorce et al. (2013) at $3.6 \mu \mathrm{m}$ using Spitzer IRAC images and Neill et al. (2014) in their study of the TFR at the WISE $W 1$ and $W 2$ bands. In each of these cases, the scatter at infrared bands was reduced by $\sim 0.1$ mag by the introduction of a color adjustment term. Empirically, color terms can effectively reduce scatter in the TFR at blueward and infrared bands, becoming minimally applicable between the $i$ and $z$ bands.

Here we evaluate correlations between TFR residuals and the various distance-independent observables that are available in our study. The features that we use to probe the type, morphology, and other general characteristics of spirals include (1) color indices calculated from optical and infrared magnitudes, (2) mean surface brightnesses of galaxies at different wave bands as probes of galaxy morphology, and (3) pseudocolors derived from the difference between the HI magnitude calculated from Equation (1) and optical/infrared magnitudes, $C_{21 \lambda}=m_{21}-m_{\lambda}$, as an estimate of the ratio of the galaxy $\mathrm{HI}$ content to its stellar mass.

These observables are not all independent of each other. In Section 4.1, we explore the correlations between these observables and the $\mathrm{HI}$ line width. The ability of quantifiable features to lower the scatter about the TFR is investigated in Section 4.2.

\subsection{Distance-independent Observables versus $H$ I Line Width}

In this section, we perform our analysis using a sample of $\sim 10,000$ spirals with high-quality photometry measurements. Distance-independent characteristics are evaluated in reference to the H I line width, the main distance-independent parameter of the TFR. We model the general relations between H I line width and other observable features using power laws that are formulated as

$$
\Omega=\omega_{0}\left(\log W_{m x}^{i}-2.5\right)+\omega_{1}
$$

where $\Omega$ can be any of the investigated features, such as color terms, surface brightness, or $C_{21 \lambda}$ parameter. Here $\omega_{0}$ and $\omega_{1}$ are the slope and zero-point of the linear relations. We minimize residuals along the $\Omega$-axis in the least-squares regression to fit the linear relation.

In the absence of the galaxy SED, color indices are effective substitutes that provide useful information on the type, morphology, stellar populations, and general characteristics of galaxies. Our spirals have measured magnitudes in seven different optical/infrared bands. Twenty-one color indices can be defined using these magnitudes. In this work, we only consider six independent color terms with reference to the $i$ band. Our choice of $i$ band is based on the relatively high image quality at that wave band; changing the reference passband does not change our conclusions.

The color terms with respect to the $i$ band are seen in Figure 12. Bigger spirals consist of redder/older stars and populate the larger line width region of the diagram. These relations steepen as the span of the wave bands that define the color term increases.

The correlations between line width and $C_{21 \lambda}$ at different passbands are plotted in Figure 14. Spirals with more positive $C_{21 \lambda}$ values (pseudoredder) have less $\mathrm{HI}$ gas relative to their stellar content. The $C_{21 \lambda}$ increases with line width, since the efficient star formation in bigger spirals consumes larger fractions of their $\mathrm{H}$ I content to form stars. The linear relations steepen as the $C_{21 \lambda}$ pseudocolor is based on longer wave bands. For a given galaxy, the $21 \mathrm{~cm}$ magnitude is a fixed parameter in the calculation of $C_{21 \lambda}$, so redder colors at larger line widths translate to steeper $C_{21 \lambda}$ slopes toward the infrared.

Figure 15 plots the mean surface brightness within the halflight radius versus $\mathrm{HI}$ line width. Surface brightness is corrected for the effect of inclination using Equation (5). More massive spirals with larger $\mathrm{H}$ I line widths tend toward higher surface brightness.

Table 7 contains the parameters of the linear fits that describe the correlations between various distance-dependent observables and $\mathrm{H}$ I line widths. 


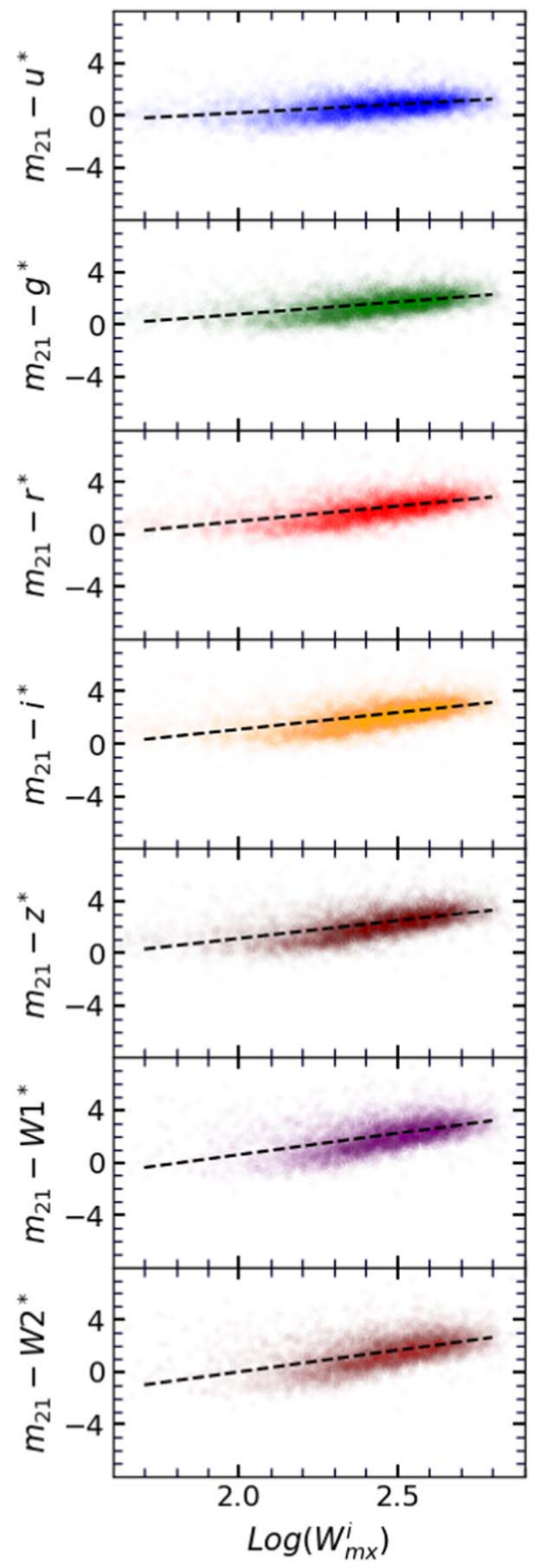

Figure 14. Similar to Figure 12 but for $m_{21}-m_{\lambda}$ vs. H I line width, where $\lambda$ represents different optical/infrared bands. The unit of the vertical axis is magnitude. The parameters of the black dashed lines are presented in Table 7.
Table 7

Parameters of the Linear Correlations between Various Distance-independent Observables and H I Line Widths as Expressed by Equation (14)

\begin{tabular}{|c|c|c|c|c|c|}
\hline $\begin{array}{l}\text { No. } \\
\text { (1) }\end{array}$ & $\begin{array}{c}\Omega^{\mathrm{a}, \mathrm{b}} \\
(2)\end{array}$ & $\begin{array}{l}\omega_{0} \\
(3)\end{array}$ & $\begin{array}{l}\omega_{1} \\
(4)\end{array}$ & $\begin{array}{c}\mathrm{rms} \\
(5)\end{array}$ & $\begin{array}{c}C(\%) \\
(6)\end{array}$ \\
\hline 1 & $u^{*}-i^{*}$ & 2.68 & 1.58 & 0.87 & 33 \\
\hline 2 & $g^{*}-i^{*}$ & 0.84 & 0.64 & 0.18 & 66 \\
\hline 3 & $r^{*}-i^{*}$ & 0.27 & 0.23 & 0.07 & 61 \\
\hline 4 & $i^{*}-z^{*}$ & 0.25 & 0.12 & 0.07 & 47 \\
\hline 5 & $i^{*}-W 1^{*}$ & 1.18 & -0.47 & 0.23 & 66 \\
\hline 6 & $i^{*}-W 2^{*}$ & 1.32 & -1.04 & 0.28 & 60 \\
\hline 7 & $m_{21}-u^{*}$ & 1.30 & 0.84 & 0.74 & 36 \\
\hline 8 & $m_{21}-g^{*}$ & 1.86 & 1.73 & 0.79 & 46 \\
\hline 9 & $m_{21}-r^{*}$ & 2.31 & 2.14 & 0.86 & 51 \\
\hline 10 & $m_{21}-i^{*}$ & 2.56 & 2.36 & 0.89 & 54 \\
\hline 11 & $m_{21}-z^{*}$ & 2.72 & 2.47 & 0.91 & 56 \\
\hline 12 & $m_{21}-W 1^{*}$ & 3.27 & 2.22 & 1.00 & 53 \\
\hline 13 & $m_{21}-W 2^{*}$ & 3.32 & 1.65 & 1.02 & 53 \\
\hline 14 & $\left\langle\mu_{u^{*}}\right\rangle_{e}$ & -0.95 & 24.30 & 1.37 & 15 \\
\hline 15 & $\left\langle\mu_{g^{*}}\right\rangle_{e}$ & -1.92 & 23.28 & 1.08 & 37 \\
\hline 16 & $\left\langle\mu_{r} *\right\rangle_{e}$ & -2.70 & 22.74 & 1.13 & 47 \\
\hline 17 & $\left\langle\mu_{i}^{*}\right\rangle_{e}$ & -3.12 & 22.48 & 1.14 & 52 \\
\hline 18 & $\left\langle\mu_{z} *\right\rangle_{e}$ & -3.45 & 22.26 & 1.16 & 55 \\
\hline 19 & $\left\langle\mu_{W 1^{*}}\right\rangle_{e}$ & -4.05 & 22.63 & 1.22 & 54 \\
\hline 20 & $\left\langle\mu_{W 2 *}\right\rangle_{e}$ & -4.06 & 23.21 & 1.41 & 49 \\
\hline
\end{tabular}

Notes. The parameter rms is the rms of the dispersion in data about the fitted lines along the axis of the corresponding parameter. In the last column, $C$ is the correlation coefficient and quantifies the strength of the relations.

a $\Omega_{1-13}$ are in mag.

${ }^{\mathrm{b}} \Omega_{14-20}$ are in mag $\operatorname{arcsec}^{-2}$.

\subsection{Linking TFR Dispersion to Distance-independent Observables}

It is shown that the scatter in the TFR can be reduced through introducing additional parameters. Sorce et al. (2013) and Neill et al. (2014) found significant correlations between deviations from the infrared TFRs and their optical-infrared color terms. In this section, we explore a range of ways to reduce scatter about the TFR at different wave bands.

The deviation of a spiral galaxy in the slope calibrator sample from the ITFR is given by Equation (9). These residuals from the ITFR are plotted against observables, and the trends of linear fits are evaluated with correlation coefficients. The strongest correlations are used to adjust magnitudes, resulting in modified ITFRs with smaller scatter. In this process, we consider two groups of observables. The first group consists of all 20 parameters listed in the second column of Table 7 . The second group considers the residuals of the distance-independent parameters from their linear relations with respect to $\mathrm{HI}$ line widths,

$$
\Delta \Omega^{i}=\Omega_{\mathrm{obs}}^{i}-\Omega,
$$

where $\Omega_{\mathrm{obs}}^{i}$ represents any of the individual distance-independent observed characteristics and $\Omega$ is calculated from the line width following Equation (14) and the parameters in Table 7.

We express the general correlations between deviation from the ITFR and galaxy observables using the linear relation

$$
\Delta M_{\lambda}^{(\Theta)}=\Phi_{\text {Slope }} \Theta+\Phi_{\mathrm{ZP}},
$$




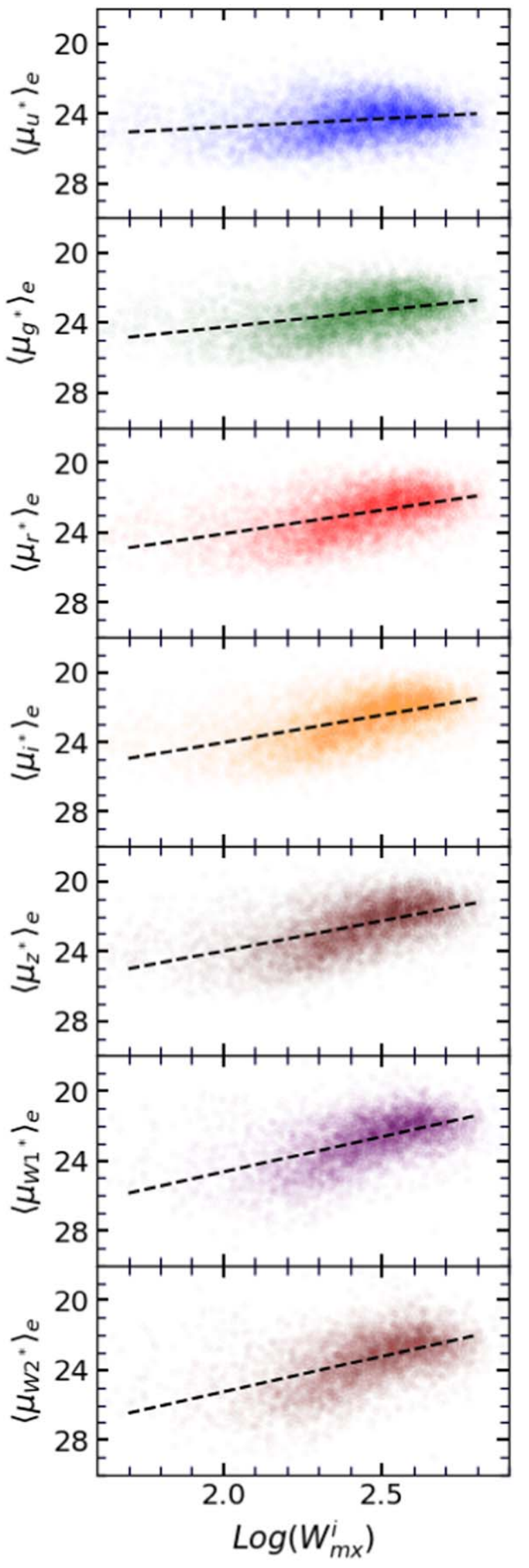

Figure 15. Similar to Figure 12 but for mean effective surface brightness, $\left\langle\mu_{\lambda^{*}}\right)_{e}^{(i)}$, vs. $\mathrm{H}$ I line width at different wave bands. The unit of the vertical axis is mag $\operatorname{arcsec}^{-2}$. The parameters of the black dashed lines are presented in Table 7.
Table 8

The Parameters of the Linear Relation between Deviations of Magnitudes from the Unadjusted TFR as Expressed in Equation (16)

\begin{tabular}{|c|c|c|c|c|}
\hline $\begin{array}{l}\text { Deviation } \\
\text { from TFR } \\
\text { (1) }\end{array}$ & $\begin{array}{c}\text { Correction } \\
\text { Parameter }(\Theta) \\
(2)\end{array}$ & $\begin{array}{c}\Phi_{\text {Slope }} \\
\text { (3) }\end{array}$ & $\begin{array}{l}\Phi_{\mathrm{ZP}} \\
(4)\end{array}$ & $\begin{array}{l}C \\
(\% \\
(5\end{array}$ \\
\hline $\begin{array}{l}u^{*} \text {-mean } \\
\text { Correlation }^{\mathrm{a}}\end{array}$ & $\begin{array}{c}u^{*}-i^{*} \\
u^{*}-W 1^{*} \\
\Delta\left(\left\langle\mu_{\left.\left.u^{*}\right\rangle_{e}\right)}\right.\right. \\
\Delta\left(m_{21}-u^{*}\right)\end{array}$ & $\begin{aligned} 0.76 & \pm 0.05 \\
0.50 & \pm 0.03 \\
-0.13 & \pm 0.02 \\
0.17 & \pm 0.03\end{aligned}$ & $\begin{array}{l}-1.13 \pm 0.07 \\
-0.48 \pm 0.04 \\
-0.21 \pm 0.04 \\
-0.29 \pm 0.05\end{array}$ & $\begin{array}{l}61 \\
58 \\
21\end{array}$ \\
\hline $\begin{array}{l}g^{*} \text {-mean } \\
\text { Correlation }\end{array}$ & $\begin{array}{c}g^{*}-i^{*} \\
g^{*}-W 1^{*} \\
i^{*}-W 1^{*}\end{array}$ & $\begin{array}{l}0.73 \pm 0.09 \\
0.35 \pm 0.05 \\
0.49 \pm 0.08\end{array}$ & $\begin{array}{r}-0.45 \pm 0.06 \\
-0.04 \pm 0.02 \\
0.26 \pm 0.05\end{array}$ & $\begin{array}{l}32 \\
25\end{array}$ \\
\hline $\begin{array}{l}r^{*} \text {-mean } \\
\text { Correlation }\end{array}$ & $\begin{array}{c}g^{*}-i^{*} \\
i^{*}-W 1^{*} \\
\Delta\left(i^{*}-W 1^{*}\right) \\
\Delta\left(\left\langle\mu_{\left.\left.r^{*}\right\rangle_{e}\right)}\right.\right. \\
\Delta\left(m_{21}-r^{*}\right)\end{array}$ & $\begin{aligned} 0.44 & \pm 0.09 \\
0.34 & \pm 0.08 \\
-0.14 & \pm 0.11 \\
0.06 & \pm 0.02 \\
-0.05 & \pm 0.02\end{aligned}$ & $\begin{aligned}-0.27 & \pm 0.06 \\
0.18 & \pm 0.05 \\
0.00 & \pm 0.02 \\
-0.01 & \pm 0.02 \\
0.01 & \pm 0.02\end{aligned}$ & $\begin{array}{l}16 \\
22 \\
23 \\
14\end{array}$ \\
\hline $\begin{array}{l}i^{*} \text {-mean } \\
\text { Correlation }\end{array}$ & $\begin{array}{c}g^{*}-i^{*} \\
i^{*}-W 1^{*} \\
\Delta\left(g^{*}-i^{*}\right) \\
\Delta\left(i^{*}-W 1^{*}\right) \\
\Delta\left(\left\langle\mu_{i^{*}}\right\rangle_{e}\right) \\
\Delta\left(m_{21}-i^{*}\right)\end{array}$ & $\begin{aligned} 0.29 & \pm 0.10 \\
0.28 & \pm 0.08 \\
-0.31 & \pm 0.14 \\
-0.24 & \pm 0.11 \\
0.06 & \pm 0.02 \\
-0.06 & \pm 0.02\end{aligned}$ & $\begin{aligned}-0.18 & \pm 0.06 \\
0.14 & \pm 0.05 \\
0.00 & \pm 0.02 \\
0.00 & \pm 0.02 \\
-0.02 & \pm 0.02 \\
0.01 & \pm 0.02\end{aligned}$ & $\begin{array}{l}14 \\
12 \\
19 \\
26 \\
24\end{array}$ \\
\hline $\begin{array}{l}z^{*} \text {-mean } \\
\text { Correlation }\end{array}$ & $\begin{array}{c}g^{*}-i^{*} \\
i^{*}-W 1^{*} \\
\Delta\left(g^{*}-i^{*}\right) \\
\Delta\left(i^{*}-W 1^{*}\right) \\
\Delta\left(\left\langle\mu_{\left.\left.z^{*}\right\rangle_{e}\right)}\right.\right. \\
\Delta\left(m_{21}-z^{*}\right)\end{array}$ & $\begin{aligned} 0.21 & \pm 0.10 \\
0.23 & \pm 0.08 \\
-0.39 & \pm 0.13 \\
-0.31 & \pm 0.11 \\
0.07 & \pm 0.02 \\
-0.08 & \pm 0.02\end{aligned}$ & $\begin{aligned}-0.14 & \pm 0.06 \\
0.11 & \pm 0.05 \\
0.00 & \pm 0.02 \\
-0.01 & \pm 0.02 \\
-0.02 & \pm 0.02 \\
0.01 & \pm 0.02\end{aligned}$ & $\begin{array}{l}11 \\
10 \\
21\end{array}$ \\
\hline $\begin{array}{l}W 1^{*} \text {-mean } \\
\text { Correlation }\end{array}$ & $\begin{array}{c}i^{*}-W 1^{*} \\
\Delta\left(i^{*}-W 1^{*}\right) \\
\Delta\left(\left\langle\mu_{\left.\left.W 1^{*}\right\rangle_{e}\right)}\right.\right. \\
\Delta\left(m_{21}-W 1^{*}\right)\end{array}$ & $\begin{aligned}-0.28 & \pm 0.09 \\
-1.08 & \pm 0.11 \\
0.16 & \pm 0.02 \\
-0.10 & \pm 0.02\end{aligned}$ & $\begin{aligned}-0.15 & \pm 0.05 \\
-0.04 & \pm 0.02 \\
-0.05 & \pm 0.02 \\
0.00 & \pm 0.02\end{aligned}$ & $\begin{array}{l}13 \\
53 \\
38\end{array}$ \\
\hline $\begin{array}{l}W 2^{*} \text {-mean } \\
\text { Correlation }\end{array}$ & $\begin{array}{c}i^{*}-W 2^{*} \\
\Delta\left(i^{*}-W 2^{*}\right) \\
\Delta\left(\left\langle\mu_{\left.W 2^{*}\right\rangle_{e}}\right)\right. \\
\Delta\left(m_{21}-W 2^{*}\right)\end{array}$ & $\begin{aligned}-0.39 & \pm 0.08 \\
-1.09 & \pm 0.09 \\
0.19 & \pm 0.02 \\
-0.12 & \pm 0.02\end{aligned}$ & $\begin{array}{l}-0.44 \pm 0.09 \\
-0.07 \pm 0.02 \\
-0.07 \pm 0.02 \\
-0.01 \pm 0.02\end{array}$ & 40 \\
\hline
\end{tabular}

Notes. In the last column, $C$ denotes the correlation strength between the deviations and the corresponding observable feature, $\Theta$.

${ }^{\mathrm{a}}$ The mean correlation is given by the unadjusted ITFR at each band, $\mathrm{TF}_{\lambda}$, where $\lambda=u, g, r, i, z, W 1$, and $W 2$, as formulated by Equation (7), with optimized parameters listed in Table 2.

where $\Delta M_{\lambda}^{(\Theta)}$ is the output of the model for deviations from the ITFR at the wave band $\lambda$, and $\Theta$ is any of the observable features, $\Omega_{\mathrm{obs}}$ or $\Delta \Omega$. Here $\Phi_{\text {Slope }}$ and $\Phi_{\mathrm{ZP}}$ are the slope and zero-point of the linear relation. Table 8 lists the parameters of the correlations between various observables, $\Omega_{\mathrm{obs}}$ or $\Delta \Omega$, and deviations from the mean unadjusted ITFRs (see Figure 8 ). To save space, this list only includes observables with the strongest correlations and a set of important features; these include mean effective surface brightness at each band, $\left\langle\mu_{\lambda^{*}}\right\rangle_{e}$; their deviations from the linear relations with line width as described by Equation (14), $\Delta\left(\left\langle\mu_{*}^{*}\right\rangle_{e}\right)$; and the $m_{21}-m_{\lambda}^{*}$ pseudocolors.

An observable $\Theta$ that is highly correlated to the ITFR residuals, $\Delta M$, offers a modified ITFR with smaller dispersion. The modified relation is constructed based on the adjusted 
Table 9

TFR Parameters Before and After Corrections

\begin{tabular}{|c|c|c|c|c|c|c|c|c|c|c|c|}
\hline \multirow{2}{*}{ Band } & \multirow{2}{*}{ Sample } & \multirow{2}{*}{$\begin{array}{l}\text { Correction } \\
\text { Parameter }\end{array}$} & \multirow{2}{*}{$\begin{array}{l}\text { TFR } \\
\text { Code }\end{array}$} & \multicolumn{3}{|c|}{ Universal Slope } & \multicolumn{3}{|c|}{ Zero-point } & \multicolumn{2}{|c|}{ Curvature } \\
\hline & & & & Ngal & Slope & $\mathrm{rms}$ & Ngal & Mag & $\overline{\mathrm{rms}}$ & Break Point & $A_{2}$ \\
\hline$u$ & OP & $\cdots$ & $\mathrm{TF}_{u}$ & 429 & $-7.03 \pm 0.17$ & 0.59 & 39 & $-19.27 \pm 0.13$ & 0.75 & $\log \left(W_{m x}^{i}\right)=2.5$ & $6.59 \pm 1.10$ \\
\hline$u$ & $\mathrm{OP}$ & $u^{*}-i^{*}$ & $\mathrm{TF}_{\mathrm{ucl}}$ & 429 & $-7.88 \pm 0.13$ & 0.49 & 39 & $-19.31 \pm 0.10$ & 0.60 & 2.5 & $5.03 \pm 0.90$ \\
\hline$u$ & $\mathrm{OP}+\mathrm{IR}$ & $u^{*}-W 1^{*}$ & $\mathrm{TF}_{\mathrm{uc} 2}$ & 400 & $-7.96 \pm 0.15$ & 0.51 & 38 & $-19.31 \pm 0.11$ & 0.61 & 2.5 & $5.90 \pm 0.91$ \\
\hline$u$ & OP & $\Delta\left(\left\langle\mu_{u}\right\rangle_{e}\right)$ & & 429 & $-7.35 \pm 0.16$ & 0.59 & 39 & $-19.33 \pm 0.12$ & 0.60 & 2.5 & $6.55 \pm 1.10$ \\
\hline$u$ & OP & $\Delta\left(m_{21}-u^{*}\right)$ & & 435 & $-7.58 \pm 0.17$ & 0.59 & 39 & $-19.43 \pm 0.13$ & 0.75 & 2.5 & $6.58 \pm 1.12$ \\
\hline$g$ & $\mathrm{OP}$ & $\ldots$ & $\mathrm{TF}_{g}$ & 430 & $-7.37 \pm 0.13$ & 0.49 & 39 & $-20.15 \pm 0.11$ & 0.62 & 2.5 & $4.18 \pm 0.90$ \\
\hline$g$ & $\mathrm{OP}$ & $g^{*}-i^{*}$ & $\mathrm{TF}_{\mathrm{gcl} 1}$ & 430 & $-8.04 \pm 0.13$ & 0.48 & 39 & $-20.18 \pm 0.10$ & 0.59 & 2.5 & $4.91 \pm 0.89$ \\
\hline$g$ & $\mathrm{OP}+\mathrm{IR}$ & $g^{*}-W 1^{*}$ & $\mathrm{TF}_{\mathrm{gc} 2}$ & 401 & $-8.00 \pm 0.14$ & 0.48 & 38 & $-20.17 \pm 0.10$ & 0.59 & 2.5 & $5.01 \pm 0.89$ \\
\hline$g$ & $\mathrm{OP}+\mathrm{IR}$ & $i^{*}-W 1^{*}$ & & 401 & $-7.85 \pm 0.14$ & 0.48 & 38 & $-20.17 \pm 0.11$ & 0.61 & 2.5 & $5.12 \pm 0.88$ \\
\hline$r$ & $\mathrm{OP}$ & $\ldots$ & $\mathrm{TF}_{r}$ & 430 & $-7.96 \pm 0.13$ & 0.49 & 39 & $-20.57 \pm 0.10$ & 0.59 & 2.5 & $4.56 \pm 0.89$ \\
\hline$r$ & $\mathrm{OP}$ & $g^{*}-i^{*}$ & & 430 & $-8.41 \pm 0.13$ & 0.49 & 39 & $-20.59 \pm 0.10$ & 0.58 & 2.5 & $5.23 \pm 0.91$ \\
\hline$r$ & $\mathrm{OP}+\mathrm{IR}$ & $i^{*}-W 1^{*}$ & & 401 & $-8.28 \pm 0.14$ & 0.49 & 38 & $-20.58 \pm 0.10$ & 0.58 & 2.5 & $5.13 \pm 0.90$ \\
\hline$r$ & $\mathrm{OP}+\mathrm{IR}$ & $\Delta\left(i^{*}-W 1^{*}\right)$ & $\mathrm{TF}_{r c}$ & 401 & $-7.89 \pm 0.14$ & 0.48 & 38 & $-20.57 \pm 0.10$ & 0.58 & 2.5 & $3.59 \pm 0.92$ \\
\hline$r$ & $\mathrm{OP}$ & $\Delta\left(\left\langle\mu_{r}\right\rangle_{e}\right)$ & $\mathrm{TF}_{r \mu}$ & 430 & $-7.89 \pm 0.13$ & 0.47 & 39 & $-20.55 \pm 0.10$ & 0.59 & 2.5 & $4.34 \pm 0.90$ \\
\hline$r$ & OP & $\Delta\left(m_{21}-r^{*}\right)$ & & 430 & $-7.81 \pm 0.13$ & 0.47 & 39 & $-20.53 \pm 0.10$ & 0.59 & 2.5 & $4.35 \pm 0.87$ \\
\hline$i$ & OP & $\ldots$ & $\mathrm{TF}_{i}$ & 430 & $-8.32 \pm 0.13$ & 0.49 & 39 & $-20.80 \pm 0.10$ & 0.59 & 2.5 & $5.34 \pm 0.91$ \\
\hline$i$ & OP & $g^{*}-i^{*}$ & & 430 & $-8.64 \pm 0.14$ & 0.49 & 39 & $-20.81 \pm 0.10$ & 0.59 & 2.5 & $5.87 \pm 0.94$ \\
\hline$i$ & $\mathrm{OP}+\mathrm{IR}$ & $i^{*}-W 1^{*}$ & & 401 & $-8.58 \pm 0.14$ & 0.49 & 38 & $-20.81 \pm 0.10$ & 0.58 & 2.5 & $5.71 \pm 0.94$ \\
\hline$i$ & $\mathrm{OP}$ & $\Delta\left(g^{*}-i^{*}\right)$ & & 430 & $-8.22 \pm 0.13$ & 0.49 & 39 & $-20.80 \pm 0.10$ & 0.59 & 2.5 & $4.68 \pm 0.89$ \\
\hline$i$ & $\mathrm{OP}+\mathrm{IR}$ & $\Delta\left(i^{*}-W 1^{*}\right)$ & $\mathrm{TF}_{i c}$ & 401 & $-8.22 \pm 0.14$ & 0.48 & 39 & $-20.81 \pm 0.10$ & 0.57 & 2.5 & $3.91 \pm 0.94$ \\
\hline$i$ & $\mathrm{OP}$ & $\Delta\left(\left\langle\mu_{i} *\right\rangle_{e}\right)$ & $\mathrm{TF}_{i \mu}$ & 430 & $-8.23 \pm 0.13$ & 0.47 & 39 & $-20.77 \pm 0.10$ & 0.59 & 2.5 & $5.03 \pm 0.92$ \\
\hline$i$ & OP & $\Delta\left(m_{21}-i^{*}\right)$ & & 435 & $-8.13 \pm 0.13$ & 0.48 & 39 & $-20.75 \pm 0.10$ & 0.59 & 2.5 & $5.01 \pm 0.89$ \\
\hline$z$ & OP & $\ldots$ & $\mathrm{TF}_{z}$ & 429 & $-8.46 \pm 0.13$ & 0.50 & 38 & $-20.89 \pm 0.10$ & 0.57 & 2.5 & $5.81 \pm 0.91$ \\
\hline$z$ & OP & $g^{*}-i^{*}$ & & 429 & $-8.69 \pm 0.13$ & 0.49 & 38 & $-20.88 \pm 0.10$ & 0.59 & 2.5 & $6.21 \pm 0.93$ \\
\hline$z$ & $\mathrm{OP}+\mathrm{IR}$ & $i^{*}-W 1^{*}$ & & 400 & $-8.60 \pm 0.14$ & 0.50 & 37 & $-20.88 \pm 0.10$ & 0.57 & 2.5 & $5.82 \pm 0.94$ \\
\hline$z$ & $\mathrm{OP}$ & $\Delta\left(g^{*}-i^{*}\right)$ & & 429 & $-8.33 \pm 0.13$ & 0.48 & 38 & $-20.88 \pm 0.10$ & 0.56 & 2.5 & $4.96 \pm 0.89$ \\
\hline$z$ & $\mathrm{OP}+\mathrm{IR}$ & $\Delta\left(i^{*}-W 1^{*}\right)$ & $\mathrm{TF}_{z c}$ & 400 & $-8.29 \pm 0.14$ & 0.47 & 37 & $-20.88 \pm 0.10$ & 0.55 & 2.5 & $3.77 \pm 0.94$ \\
\hline$z$ & $\mathrm{OP}$ & $\Delta\left(\left\langle\mu_{z}\right\rangle_{e}\right)$ & $\mathrm{TF}_{z \mu}$ & 429 & $-8.35 \pm 0.13$ & 0.47 & 38 & $-20.86 \pm 0.10$ & 0.57 & 2.5 & $3.88 \pm 0.95$ \\
\hline$z$ & OP & $\Delta\left(m_{21}-z^{*}\right)$ & & 429 & $-8.22 \pm 0.13$ & 0.47 & 38 & $-20.81 \pm 0.10$ & 0.57 & 2.5 & $5.34 \pm 0.88$ \\
\hline$W 1$ & IR & $\ldots$ & $\mathrm{TF}_{W 1}$ & 584 & $-9.47 \pm 0.14$ & 0.58 & 64 & $-20.36 \pm 0.07$ & 0.60 & 2.4 & $3.81 \pm 0.42$ \\
\hline$W 1$ & $\mathrm{OP}+\mathrm{IR}$ & $i^{*}-W 1^{*}$ & & 401 & $-9.21 \pm 0.15$ & 0.54 & 38 & $-20.30 \pm 0.10$ & 0.60 & 2.4 & $3.41 \pm 0.44$ \\
\hline$W 1$ & $\mathrm{OP}+\mathrm{IR}$ & $\Delta\left(i^{*}-W 1^{*}\right)$ & $\mathrm{TF}_{W 1 c}$ & 401 & $-9.27 \pm 0.14$ & 0.47 & 38 & $-20.29 \pm 0.10$ & 0.56 & 2.4 & $1.22 \pm 0.40$ \\
\hline$W 1$ & IR & $\Delta\left(\left\langle\mu_{\left.W 1^{*}\right\rangle_{e}}\right)\right.$ & $\mathrm{TF}_{W 1 \mu}$ & 584 & $-9.15 \pm 0.13$ & 0.51 & 64 & $-20.26 \pm 0.07$ & 0.57 & 2.4 & $2.55 \pm 0.40$ \\
\hline$W 1$ & IR & $\Delta\left(m_{21}-W 1^{*}\right)$ & & 584 & $-9.12 \pm 0.13$ & 0.53 & 64 & $-20.29 \pm 0.08$ & 0.58 & 2.4 & $3.35 \pm 0.40$ \\
\hline$W 2$ & IR & $\ldots$ & $\mathrm{TF}_{W 2}$ & 584 & $-9.66 \pm 0.15$ & 0.62 & 64 & $-19.76 \pm 0.08$ & 0.65 & 2.4 & $4.42 \pm 0.43$ \\
\hline$W 2$ & $\mathrm{OP}+\mathrm{IR}$ & $i^{*}-W 2^{*}$ & & 401 & $-9.14 \pm 0.15$ & 0.55 & 38 & $-19.70 \pm 0.11$ & 0.63 & 2.4 & $3.47 \pm 0.43$ \\
\hline$W 2$ & $\mathrm{OP}+\mathrm{IR}$ & $\Delta\left(i^{*}-W 2^{*}\right)$ & $\mathrm{TF}_{W 2 c}$ & 401 & $-9.40 \pm 0.14$ & 0.47 & 38 & $-19.70 \pm 0.10$ & 0.56 & 2.4 & $1.13 \pm 0.41$ \\
\hline$W 2$ & IR & $\Delta\left(\left\langle\mu_{\left.W 2^{*}\right\rangle_{e}}\right)\right.$ & $\mathrm{TF}_{W 2 \mu}$ & 584 & $-9.18 \pm 0.13$ & 0.52 & 64 & $-19.62 \pm 0.07$ & 0.60 & 2.4 & $2.67 \pm 0.39$ \\
\hline$W 2$ & IR & $\Delta\left(m_{21}-W 1^{*}\right)$ & & 584 & $-9.22 \pm 0.14$ & 0.56 & 64 & $-19.67 \pm 0.08$ & 0.62 & 2.4 & $3.79 \pm 0.41$ \\
\hline
\end{tabular}

apparent magnitudes that are given as

$$
C_{\lambda}(\Theta)=m_{\lambda}^{*}-\Delta M_{\lambda}^{(\Theta)},
$$

where $\Delta M_{\lambda}^{(\Theta)}$ is calculated based on the observable parameter, $\Theta$, following Equation (16). The same algorithm as explained in Section 3 is pursued to determine the slope and zeropoint of the revised ITFR that has the form $\mathcal{M}_{C_{\lambda}(\Theta)}^{*}=$ Slope $\left(\log W_{m x}^{i}-2.5\right)+$ ZP. In Table 9, we present the parameters of our ITFR fits for unadjusted and adjusted magnitudes. In this table, the unadjusted relations are denoted by $\mathrm{TF}_{\lambda}$ codes. We give specific attention to interesting cases where either the rms scatter of the relation is significantly reduced after adjusting magnitudes or the correction parameter is highly correlated with the dispersion of the original relation according to Table 8 . These cases are attributed unique codes for further discussion.

Following the procedure discussed in Section 3.7, we fit a second-degree polynomial to the bright end of the luminosityline width correlations for each of the adjusted and unadjusted cases. The last two columns of Table 9 list the parameters of the fitted curves as formulated in Equation (13).

The distance modulus of a spiral galaxy with the absolute pseudomagnitude, $\mathcal{M}_{C_{\lambda}(\Theta)}^{*}$, that is given by the modified ITFR is derived as

$$
\mu_{C_{\lambda}(\Theta)}=C_{\lambda}(\Theta)-\mathcal{M}_{C_{\lambda}(\Theta)}^{*},
$$

where $C_{\lambda}(\Theta)$ is the galaxy apparent pseudomagnitude that is determined by Equation (17). 

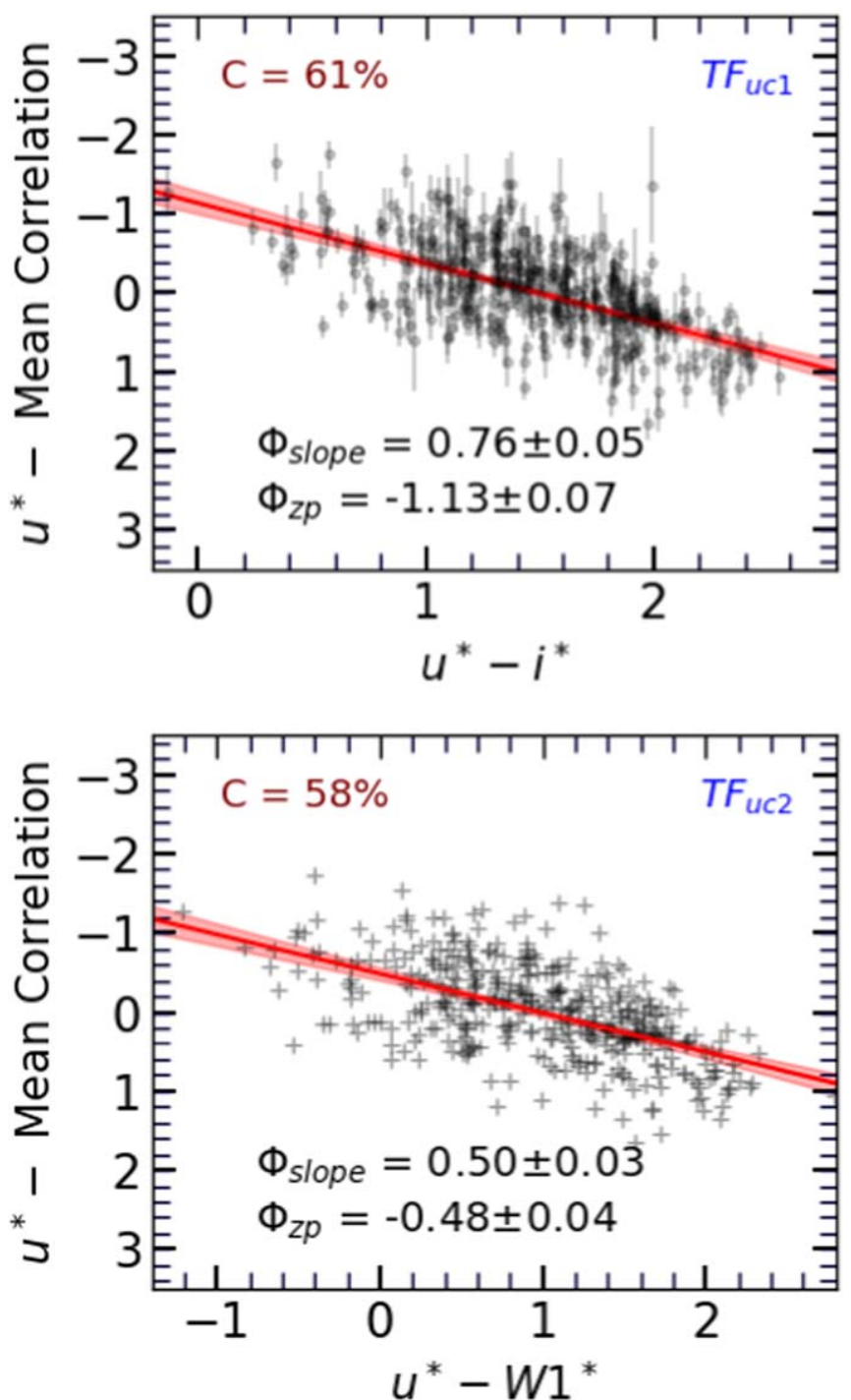

Figure 16. Deviations from the mean unadjusted ITFRs at the $u$ band vs. color. Each black point represents a galaxy. Dominant errors are along the vertical axis. The best linear relation is derived by least-squares regression incorporating the vertical uncertainties. Vertical error bars are displayed in the top panel as an example. The $95 \%$ confidence envelope is displayed about each fitted line. In the top left corner of each panel, $C$ is the correlation coefficient of the plotted parameters as listed in Table 8. In the top right corner is the code for the corresponding corrected ITFR.

\subsection{Modified ITFRs at $\mathrm{u}$ Band}

Among the correction parameters presented in the $u$-band section of Table 8 , the $u^{*}-i^{*}$ and $u^{*}-W 1^{*}$ colors are highly correlated with deviations from the mean $u$-band ITFR with correlation factors of $61 \%$ and $58 \%$, respectively. These correlations are illustrated in Figure 16, together with the best-fit linear relations described by Equation (16). The parameters of the fitted red lines are derived following the least-squares optimization along the vertical axis. Our fitting procedure ignores the horizontal errors, because they are significantly smaller than the vertical uncertainties and relatively the same for all spirals. These two linear relations are

$$
\begin{aligned}
\Delta M_{u}^{\left(u^{*}-i^{*}\right)}= & -(1.13 \pm 0.07) \\
& +(0.76 \pm 0.05)\left(u^{*}-i^{*}\right),
\end{aligned}
$$
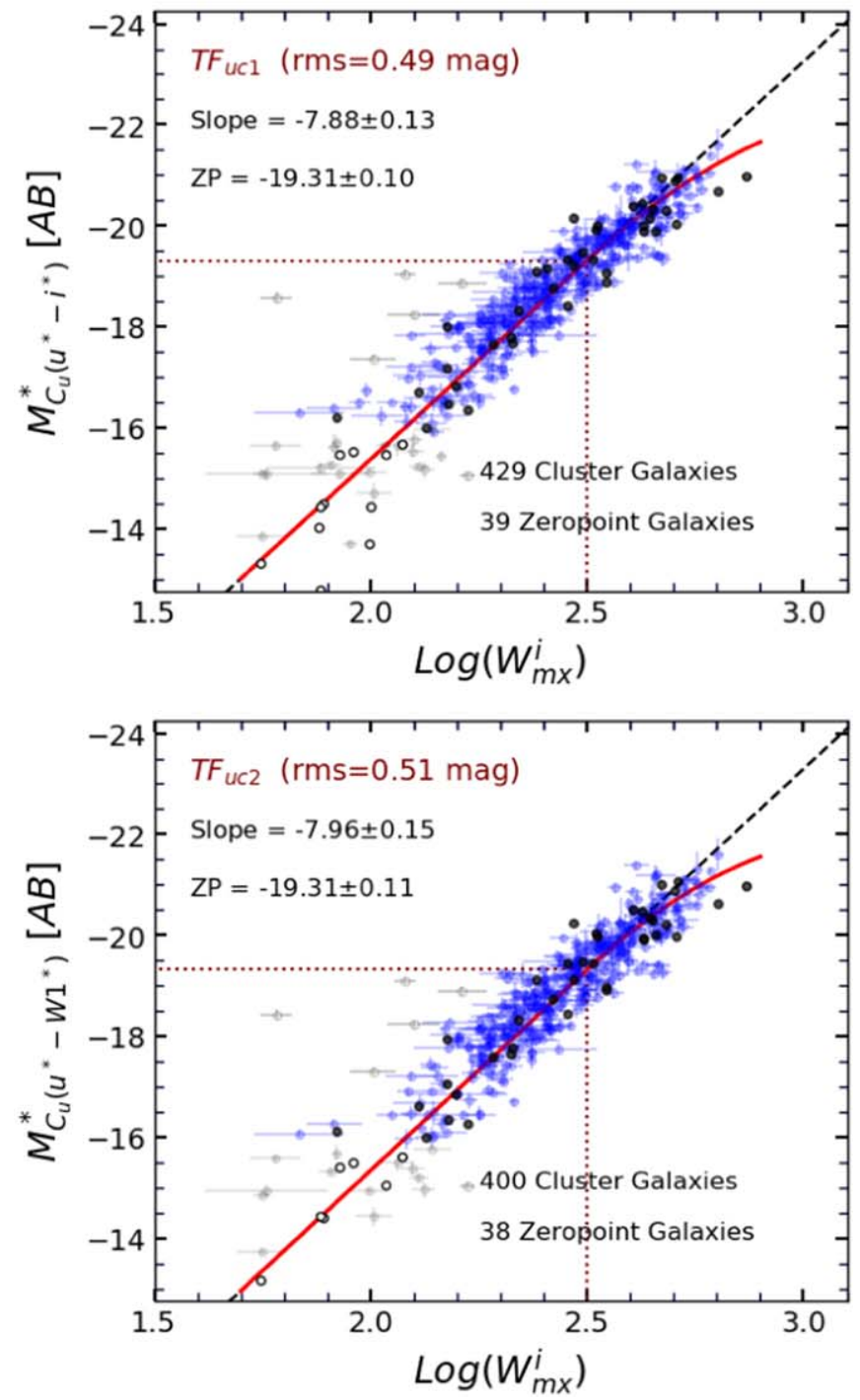

Figure 17. Unadjusted and revised ITFR relations. Top: unadjusted ITFR at the $u$ band $\left(\mathrm{TF}_{u}\right)$. This panel is the same as the top left panel of Figure 8. The rms of vertical residuals about the relation is $0.59 \mathrm{mag}$. Middle: modified ITFR after adjusting magnitudes using the $u^{*}-i^{*}$ color $\left(\mathrm{TF}_{u c 1}\right)$ with an rms scatter of 0.49 mag. Bottom: modified ITFR after adjusting magnitudes based on the $u^{*}-W 1^{*}$ color $\left(\mathrm{TF}_{u c 2}\right)$ with an rms scatter of $0.51 \mathrm{mag}$. 

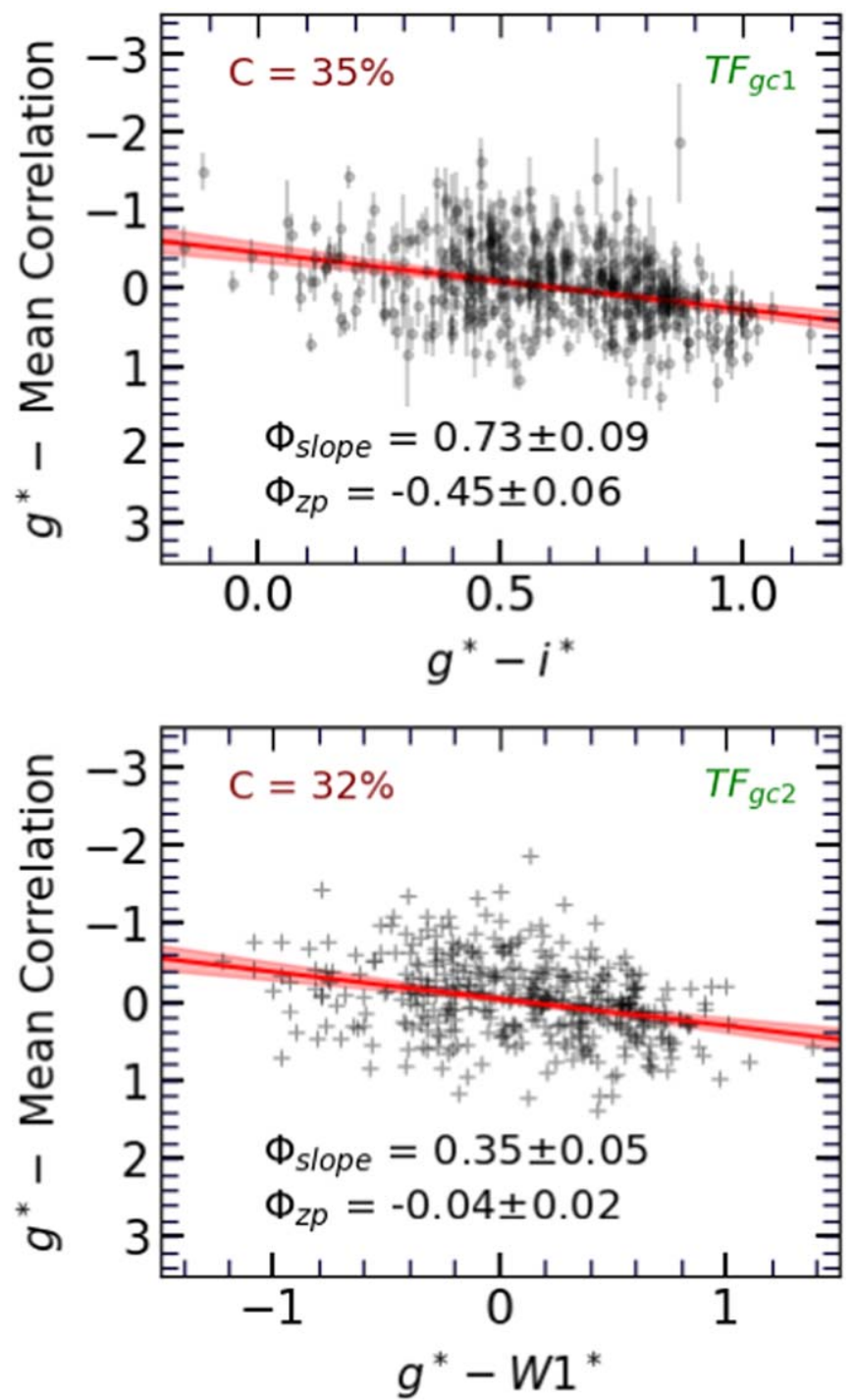

Figure 18. Same as Figure 16 but for deviations from the mean unadjusted ITFRs at the $g$ band vs. color.

$$
\begin{aligned}
\Delta M_{u}^{\left(u^{*}-W 1^{*}\right)}= & -(0.48 \pm 0.04) \\
& +(0.50 \pm 0.03)\left(u^{*}-W 1^{*}\right),
\end{aligned}
$$

where the parameter $\Theta$ in Equation (16) is replaced by $\left(u^{*}-i^{*}\right)$ and $\left(u^{*}-W 1^{*}\right)$ color terms. The revised ITFR relations are then derived based on the $u$-band pseudomagnitudes, $C_{u}(\Theta)$, through

$$
\begin{gathered}
C_{u}\left(u^{*}-i^{*}\right)=u^{*}-\Delta M_{u}^{\left(u^{*}-i^{*}\right)}, \\
C_{u}\left(u^{*}-W 1^{*}\right)=u^{*}-\Delta M_{u}^{\left(u^{*}-W 1^{*}\right)} .
\end{gathered}
$$

The corresponding modified ITFRs have the form

$$
\begin{aligned}
\mathcal{M}_{C_{u}\left(u^{*}-i^{*}\right)}^{*}= & -(19.31 \pm 0.10) \\
& -(7.88 \pm 0.13)\left(\log W_{m x}^{i}-2.5\right), \\
\mathcal{M}_{C_{u}\left(u^{*}-W 1^{*}\right)}^{*}= & -(19.31 \pm 0.11) \\
& -(7.96 \pm 0.15)\left(\log W_{m x}^{i}-2.5\right) .
\end{aligned}
$$

We assign the $\mathrm{TF}_{u c 1}$ and $\mathrm{TF}_{u c 2}$ codes to the revised ITFRs presented in Equations 21(a) and (b), respectively. These relations are illustrated in the middle and bottom panels of
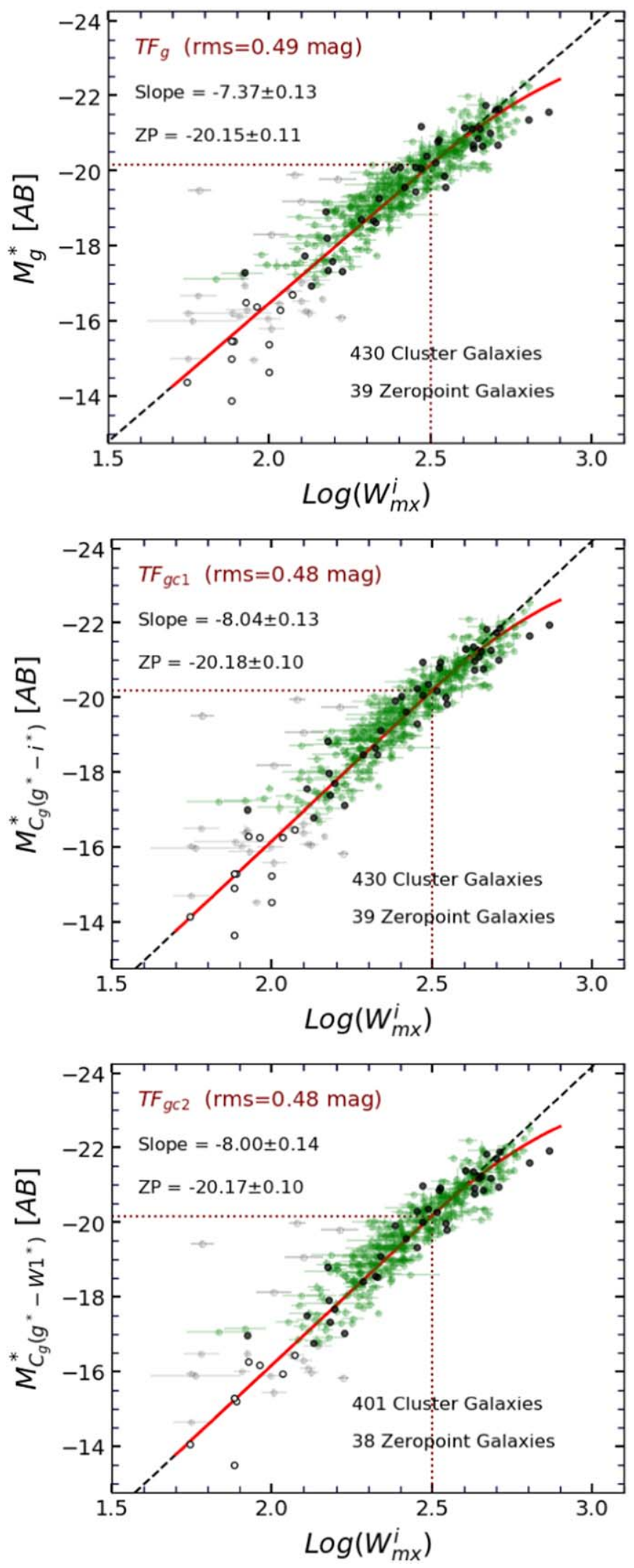

Figure 19. Same as Figure 17 for the $g$ band.

Figure 17. For comparison, the top panel of Figure 17 shows the unadjusted ITFR constructed using $u$ magnitudes $\left(\mathrm{TF}_{u}\right)$. In this figure, the partially curved relations are illustrated in solid red, with the linear part below the break point at $\log W_{m x}^{i}=2.5$ 

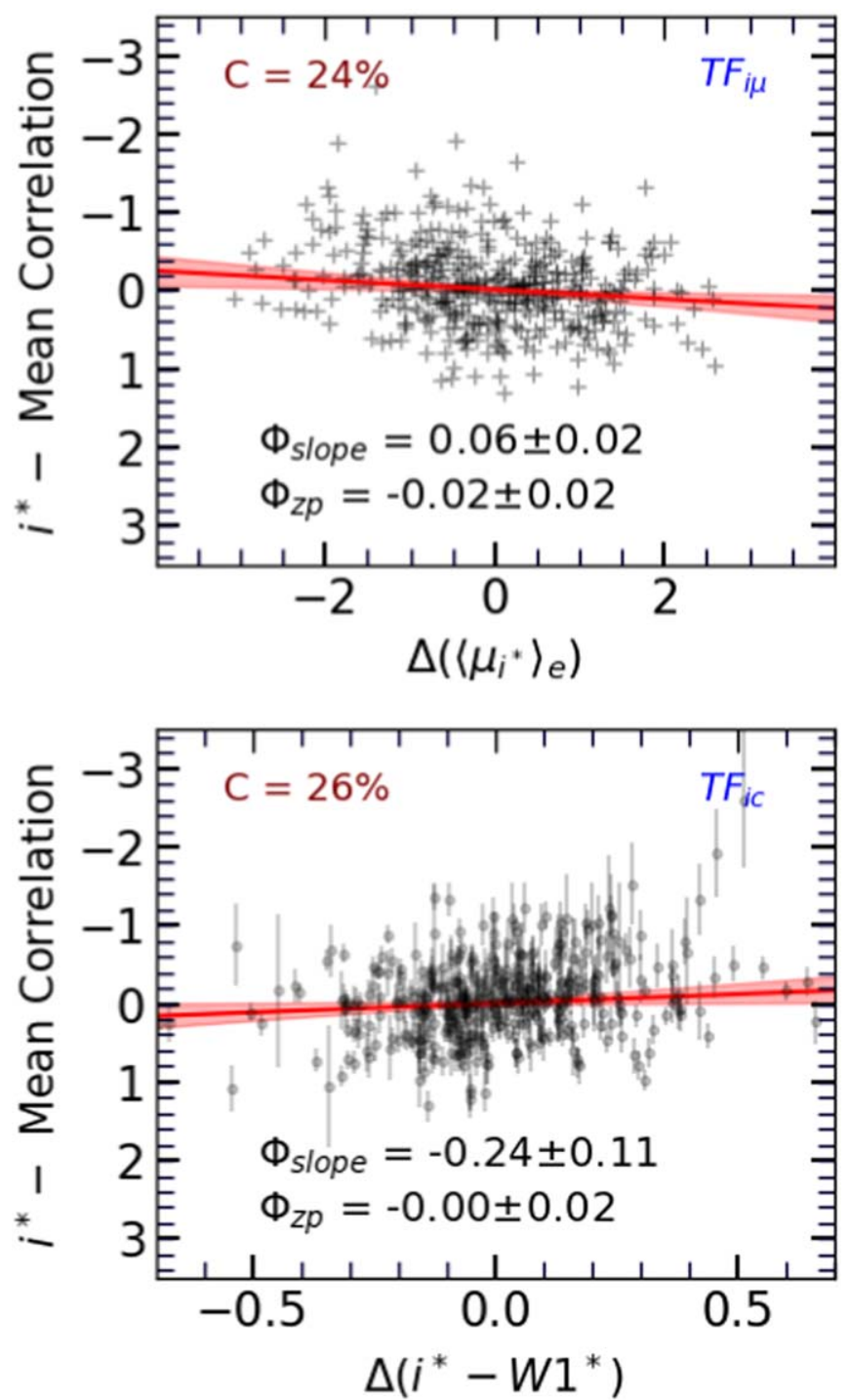

Figure 20. Deviations from the mean ITFR at the $i$ band as functions of surface brightness and color.

and the polynomial fits to data points brightward of the break point. Dashed black lines are the continuations of the linear ITFRs. To evaluate the performance of the models, we compare the scatters about the linear magnitude-line width relations. The rms of the residuals from the unadjusted $\mathrm{TF}_{u}$ of $0.59 \mathrm{mag}$ is significantly reduced to 0.49 and $0.51 \mathrm{mag}$ for $\mathrm{TF}_{u c 1}$ and $\mathrm{TF}_{u c 2}$, respectively. The $\mathrm{TF}_{u c 2}$ model requires the availability of WISE photometry that is only present for 400 out of 429 galaxies with $u$-band photometry. However, we do not expect that the lack of 29 galaxies from the $\mathrm{TF}_{u c 2}$ sample has a significant influence on our final conclusion.

The $u$ band is sensitive to populations of blue stars and starforming regions, whereas the majority of the baryonic mass in spiral galaxies resides in older stellar populations that dominate at longer wave bands. Color terms reduce scatter by providing information about older stellar populations that are subdominant in the $u$-band imaging.

\subsection{Modified ITFRs at $\mathrm{g}$ Band}

Resembling the $u$ band, it is seen in Table 8 that deviations from the mean unadjusted ITFRs at the $g$ band are most
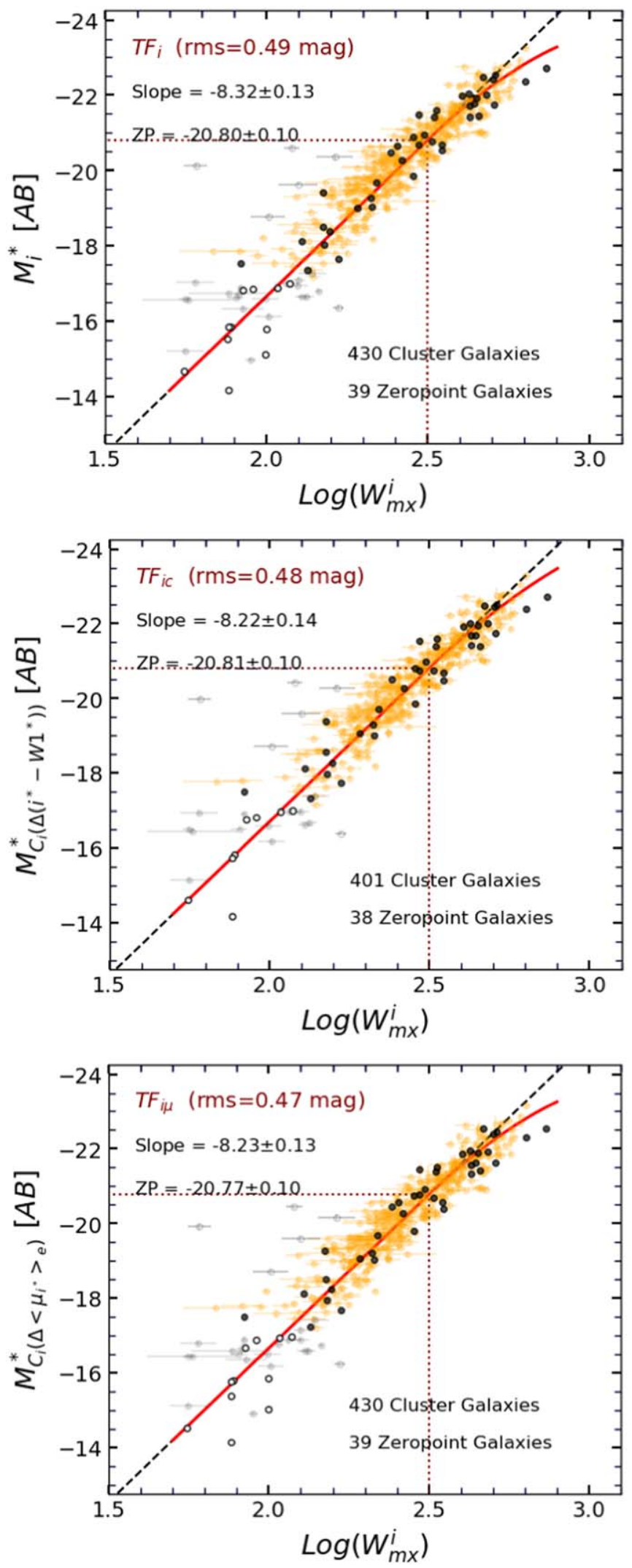

Figure 21. Same as Figure 17 for the $i$ band.

correlated with $g^{*}-i^{*}$ and $g^{*}-W 1^{*}$ color indices, with correlation factors of $35 \%$ and $32 \%$, respectively. These correlations are shown in Figure 16. The parameters of the 

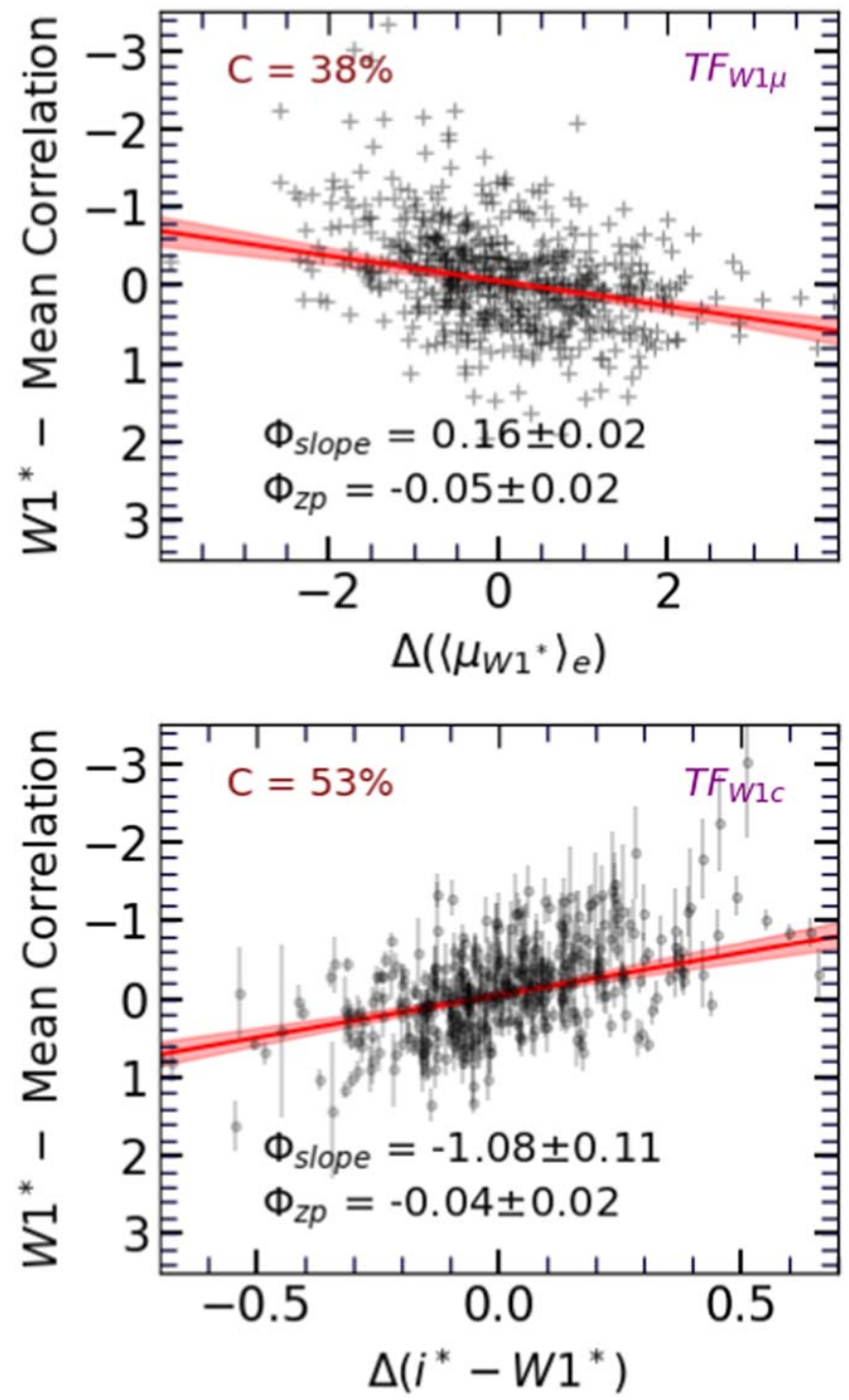

Figure 22. Same as Figure 20 but for deviations from the mean ITFR at the infrared $W 1$ band.

two linear fits required to adjust the $g$-band magnitudes are presented in Table 8 . The corresponding adjusted ITFRs based on the $g$-band pseudomagnitudes are given as

$$
\begin{aligned}
\mathcal{M}_{C_{g}\left(g^{*}-i^{*}\right)}^{*} & -(20.18 \pm 0.10) \\
& -(8.04 \pm 0.13)\left(\log W_{m x}^{i}-2.5\right), \\
\mathcal{M}_{C_{g}\left(g^{*}-W 1^{*}\right)}^{*} & -(20.17 \pm 0.10) \\
& -(8.00 \pm 0.14)\left(\log W_{m x}^{i}-2.5\right) .
\end{aligned}
$$

The modified ITFR models that are represented by Equations 22(a) and (b) are labeled with $\mathrm{TF}_{g c 1}$ and $\mathrm{TF}_{g c 2}$ codes (also see Table 9). The fits are illustrated in Figure 19.

\subsection{Modified ITFRs at $\mathrm{r}, \mathrm{i}$, and $\mathrm{z}$ Bands}

The rms scatter in unadjusted ITFRs at the $r, i$, and $z$ bands is similar. Exploring Tables 8 and 9 jointly reveals the observables
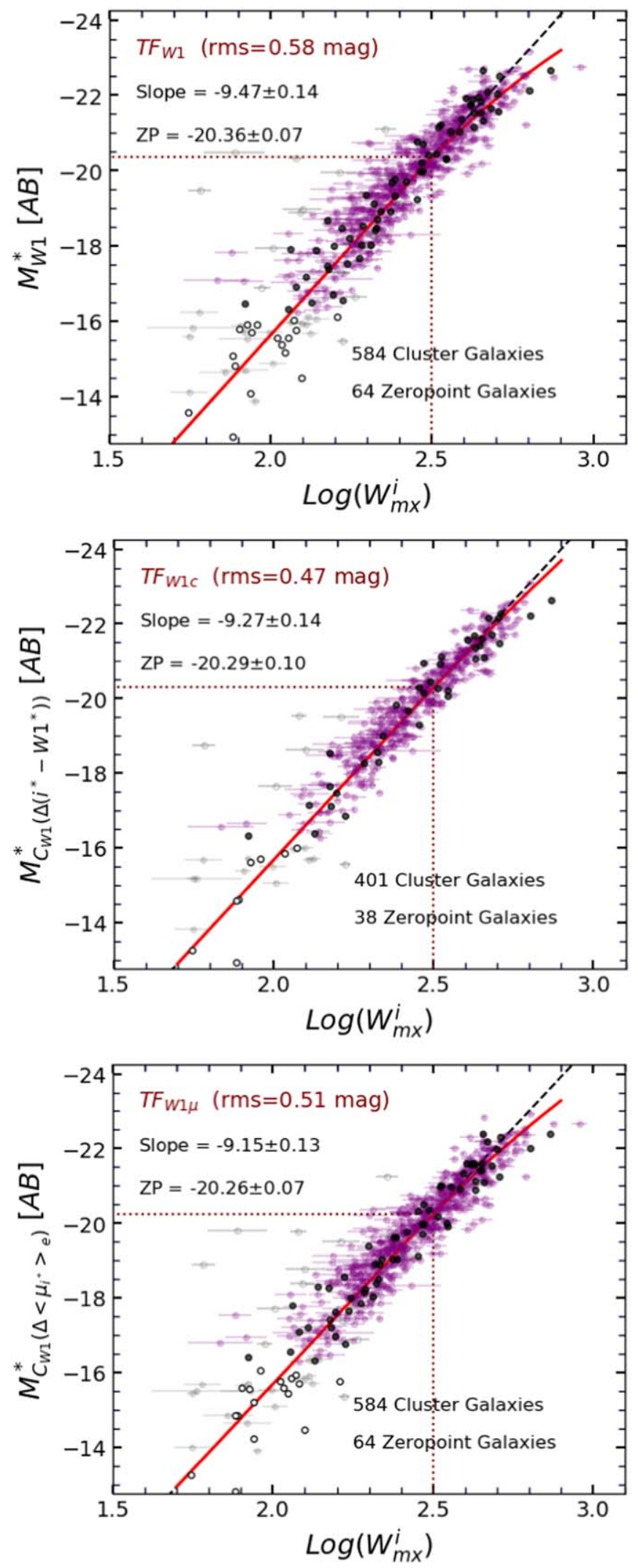

Figure 23. Same as Figure 17 for the $W 1$ band. Red solid lines display the partially curved ITFRs that deviate from the linear relation for galaxies with $\mathrm{H} \mathrm{I}$ line widths larger than $\log \left(W_{m x}^{i}\right)=2.4$. 
Table 10

Cluster Distances and $H_{0}$ Data

\begin{tabular}{|c|c|c|c|c|c|c|c|c|c|c|}
\hline Cluster & $\begin{array}{c}V_{\text {mod }} \\
\mathrm{km} \mathrm{s}^{-1} \\
(2)\end{array}$ & $\begin{array}{l}\text { TFR } \\
\text { Band } \\
(3)\end{array}$ & $\begin{array}{l}\text { TFR } \\
\text { Model } \\
(4)\end{array}$ & $\begin{array}{l}\text { Ngal } \\
(5)\end{array}$ & $\begin{array}{c}\mathrm{DM}_{o} \\
\mathrm{mag} \\
(6)\end{array}$ & $\begin{array}{l}\text { Bias } \\
\text { mag } \\
(7)\end{array}$ & $\begin{array}{c}\Delta \mathrm{DM} \\
\mathrm{mag} \\
(8)\end{array}$ & $\begin{array}{c}\mathrm{DM}_{c} \\
\mathrm{mag} \\
(9)\end{array}$ & $\begin{array}{c}D \\
\mathrm{Mpc} \\
(10)\end{array}$ & $\begin{array}{c}V_{\mathrm{mod}} / D \\
\mathrm{~km} \mathrm{~s}^{-1} \mathrm{Mpc}^{-1} \\
(11)\end{array}$ \\
\hline \multirow[t]{21}{*}{ Virgo } & \multirow[t]{21}{*}{$1516 \pm 45$} & $u$ & $\mathrm{TF}_{u}$ & 23 & 31.01 & 0.000 & & $31.01 \pm 0.12$ & $15.92 \pm 0.88$ & $95.21 \pm 5.97$ \\
\hline & & $u$ & $\mathrm{TF}_{\mathrm{uc} 1}$ & 23 & 30.95 & 0.000 & & $30.95 \pm 0.09$ & $15.49 \pm 0.64$ & $97.88 \pm 4.99$ \\
\hline & & $u$ & $\mathrm{TF}_{\mathrm{uc} 2}$ & 23 & 30.96 & 0.000 & & $30.96 \pm 0.09$ & $15.56 \pm 0.64$ & $97.43 \pm 4.97$ \\
\hline & & $g$ & $\mathrm{TF}_{g}$ & 24 & 31.05 & 0.001 & & $31.05 \pm 0.09$ & $16.23 \pm 0.67$ & $93.42 \pm 4.76$ \\
\hline & & $g$ & $\mathrm{TF}_{\mathrm{gc} 1}$ & 24 & 30.98 & 0.000 & & $30.98 \pm 0.09$ & $15.70 \pm 0.65$ & $96.54 \pm 4.92$ \\
\hline & & $g$ & $\mathrm{TF}_{\mathrm{gc} 2}$ & 24 & 31.00 & 0.000 & & $31.00 \pm 0.09$ & $15.85 \pm 0.66$ & $95.65 \pm 4.88$ \\
\hline & & $r$ & $\mathrm{TF}_{r}$ & 24 & 30.95 & 0.002 & & $30.95 \pm 0.08$ & $15.50 \pm 0.57$ & $97.81 \pm 4.63$ \\
\hline & & $r$ & $\mathrm{TF}_{r c}$ & 24 & 30.96 & 0.001 & & $30.96 \pm 0.08$ & $15.56 \pm 0.57$ & $97.41 \pm 4.61$ \\
\hline & & $r$ & $\mathrm{TF}_{r \mu}$ & 24 & 30.93 & 0.001 & & $30.93 \pm 0.08$ & $15.35 \pm 0.57$ & $98.75 \pm 4.67$ \\
\hline & & $i$ & $\mathrm{TF}_{i}$ & 24 & 30.87 & 0.001 & & $30.87 \pm 0.09$ & $14.94 \pm 0.62$ & $101.49 \pm 5.17$ \\
\hline & & $i$ & $\mathrm{TF}_{i c}$ & 24 & 30.90 & 0.001 & & $30.90 \pm 0.08$ & $15.14 \pm 0.56$ & $100.11 \pm 4.74$ \\
\hline & & $i$ & $\mathrm{TF}_{i \mu}$ & 24 & 30.86 & 0.001 & & $30.86 \pm 0.08$ & $14.87 \pm 0.55$ & $101.97 \pm 4.82$ \\
\hline & & $z$ & $\mathrm{TF}_{z}$ & 23 & 30.91 & 0.002 & & $30.91 \pm 0.09$ & $15.22 \pm 0.63$ & $99.63 \pm 5.08$ \\
\hline & & $z$ & $\mathrm{TF}_{z c}$ & 23 & 30.96 & 0.001 & & $30.96 \pm 0.08$ & $15.57 \pm 0.57$ & $97.40 \pm 4.61$ \\
\hline & & $z$ & $\mathrm{TF}_{z \mu}$ & 23 & 30.90 & 0.000 & & $30.90 \pm 0.08$ & $15.14 \pm 0.56$ & $100.15 \pm 4.74$ \\
\hline & & $W 1$ & $\mathrm{TF}_{W 1}$ & 24 & 30.80 & 0.000 & & $30.80 \pm 0.10$ & $14.46 \pm 0.67$ & $104.86 \pm 5.75$ \\
\hline & & $W 1$ & $\mathrm{TF}_{W 1 c}$ & 24 & 30.86 & 0.001 & 0.04 & $30.90 \pm 0.08$ & $15.14 \pm 0.56$ & $100.11 \pm 4.74$ \\
\hline & & $W 1$ & $\mathrm{TF}_{\mathrm{W} 1 \mu}$ & 24 & 30.82 & -0.001 & 0.11 & $30.93 \pm 0.09$ & $15.34 \pm 0.64$ & $98.83 \pm 5.04$ \\
\hline & & $W 2$ & $\mathrm{TF}_{W 2}$ & 24 & 30.79 & 0.001 & & $30.79 \pm 0.11$ & $14.39 \pm 0.73$ & $105.33 \pm 6.18$ \\
\hline & & $W 2$ & $\mathrm{TF}_{W 2 c}$ & 24 & 30.86 & 0.000 & 0.06 & $30.92 \pm 0.08$ & $15.28 \pm 0.56$ & $99.23 \pm 4.69$ \\
\hline & & $W 2$ & $\mathrm{TF}_{W 2 \mu}$ & 24 & 30.81 & -0.001 & 0.17 & $30.98 \pm 0.09$ & $15.70 \pm 0.65$ & $96.58 \pm 4.92$ \\
\hline \multirow[t]{21}{*}{ Ursa Major } & \multirow[t]{21}{*}{$1141 \pm 13$} & $u$ & $\mathrm{TF}_{u}$ & 36 & 31.46 & 0.000 & & $31.46 \pm 0.09$ & $19.59 \pm 0.81$ & $58.25 \pm 2.50$ \\
\hline & & $u$ & $\mathrm{TF}_{\mathrm{uc} 1}$ & 36 & 31.36 & 0.000 & & $31.36 \pm 0.07$ & $18.71 \pm 0.60$ & $60.99 \pm 2.09$ \\
\hline & & $u$ & $\mathrm{TF}_{\mathrm{uc} 2}$ & 36 & 31.34 & 0.000 & & $31.34 \pm 0.07$ & $18.54 \pm 0.60$ & $61.56 \pm 2.10$ \\
\hline & & $g$ & $\mathrm{TF}_{g}$ & 36 & 31.45 & 0.003 & & $31.45 \pm 0.07$ & $19.52 \pm 0.63$ & $58.45 \pm 2.00$ \\
\hline & & $g$ & $\mathrm{TF}_{\mathrm{gcl}}$ & 36 & 31.38 & 0.002 & & $31.38 \pm 0.07$ & $18.90 \pm 0.61$ & $60.37 \pm 2.06$ \\
\hline & & $g$ & $\mathrm{TF}_{\mathrm{gc} 2}$ & 36 & 31.38 & 0.001 & & $31.38 \pm 0.07$ & $18.89 \pm 0.61$ & $60.40 \pm 2.07$ \\
\hline & & $r$ & $\mathrm{TF}_{r}$ & 36 & 31.36 & 0.002 & & $31.36 \pm 0.07$ & $18.73 \pm 0.60$ & $60.93 \pm 2.08$ \\
\hline & & $r$ & $\mathrm{TF}_{r c}$ & 36 & 31.38 & -0.001 & & $31.38 \pm 0.07$ & $18.88 \pm 0.61$ & $60.45 \pm 2.07$ \\
\hline & & $r$ & $\mathrm{TF}_{r \mu}$ & 36 & 31.35 & 0.000 & & $31.35 \pm 0.07$ & $18.62 \pm 0.60$ & $61.27 \pm 2.09$ \\
\hline & & $i$ & $\mathrm{TF}_{i}$ & 36 & 31.28 & 0.002 & & $31.28 \pm 0.07$ & $18.05 \pm 0.58$ & $63.21 \pm 2.16$ \\
\hline & & $i$ & $\mathrm{TF}_{i c}$ & 36 & 31.31 & 0.003 & & $31.31 \pm 0.07$ & $18.30 \pm 0.59$ & $62.33 \pm 2.13$ \\
\hline & & $i$ & $\mathrm{TF}_{i \mu}$ & 36 & 31.28 & 0.002 & & $31.28 \pm 0.07$ & $18.05 \pm 0.58$ & $63.23 \pm 2.16$ \\
\hline & & $z$ & $\mathrm{TF}_{z}$ & 36 & 31.31 & 0.003 & & $31.31 \pm 0.07$ & $18.31 \pm 0.59$ & $62.31 \pm 2.13$ \\
\hline & & $z$ & $\mathrm{TF}_{z c}$ & 36 & 31.36 & 0.002 & & $31.36 \pm 0.07$ & $18.72 \pm 0.60$ & $60.95 \pm 2.08$ \\
\hline & & $z$ & $\mathrm{TF}_{z \mu}$ & 36 & 31.31 & -0.002 & & $31.31 \pm 0.07$ & $18.26 \pm 0.59$ & $62.48 \pm 2.14$ \\
\hline & & $W 1$ & $\mathrm{TF}_{W 1}$ & 36 & 31.15 & 0.000 & & $31.15 \pm 0.08$ & $16.98 \pm 0.63$ & $67.19 \pm 2.59$ \\
\hline & & $W 1$ & $\mathrm{TF}_{W 1 c}$ & 36 & 31.26 & 0.000 & 0.04 & $31.30 \pm 0.07$ & $18.20 \pm 0.59$ & $62.69 \pm 2.14$ \\
\hline & & $W 1$ & $\mathrm{TF}_{W 1 \mu}$ & 36 & 31.15 & 0.000 & 0.11 & $31.26 \pm 0.07$ & $17.86 \pm 0.58$ & $63.87 \pm 2.18$ \\
\hline & & $W 2$ & $\mathrm{TF}_{W 2}$ & 36 & 31.11 & 0.001 & & $31.11 \pm 0.08$ & $16.68 \pm 0.61$ & $68.41 \pm 2.64$ \\
\hline & & $W 2$ & $\mathrm{TF}_{W 2 c}$ & 36 & 31.27 & 0.000 & 0.06 & $31.33 \pm 0.07$ & $18.45 \pm 0.59$ & $61.83 \pm 2.11$ \\
\hline & & $W 2$ & $\mathrm{TF}_{W 2 \mu}$ & 36 & 31.11 & -0.000 & 0.17 & $31.28 \pm 0.08$ & $18.03 \pm 0.66$ & $63.30 \pm 2.44$ \\
\hline \multirow[t]{4}{*}{ Fornax } & \multirow[t]{4}{*}{$1383 \pm 32$} & $W 1$ & $\mathrm{TF}_{W 1}$ & 17 & 31.21 & 0.002 & & $31.21 \pm 0.14$ & $17.48 \pm 1.13$ & $79.13 \pm 5.42$ \\
\hline & & $W 1$ & $\mathrm{TF}_{W 1 \mu}$ & 17 & 31.19 & -0.003 & 0.11 & $31.30 \pm 0.12$ & $18.17 \pm 1.00$ & $76.11 \pm 4.56$ \\
\hline & & $W 2$ & $\mathrm{TF}_{W 2}$ & 17 & 31.18 & 0.001 & & $31.18 \pm 0.16$ & $17.23 \pm 1.27$ & $80.27 \pm 6.20$ \\
\hline & & $W 2$ & $\mathrm{TF}_{W 2 \mu}$ & 17 & 31.16 & -0.005 & 0.17 & $31.32 \pm 0.13$ & $18.40 \pm 1.10$ & $75.15 \pm 4.82$ \\
\hline \multirow[t]{12}{*}{ Pegasus } & \multirow[t]{12}{*}{$3249 \pm 61$} & $u$ & $\mathrm{TF}_{u}$ & 24 & 33.42 & 0.005 & & $33.42 \pm 0.13$ & $48.41 \pm 2.90$ & $67.12 \pm 4.21$ \\
\hline & & $u$ & $\mathrm{TF}_{\mathrm{uc} 1}$ & 24 & 33.34 & 0.003 & & $33.34 \pm 0.11$ & $46.62 \pm 2.36$ & $69.69 \pm 3.77$ \\
\hline & & $u$ & $\mathrm{TF}_{\mathrm{uc} 2}$ & 22 & 33.36 & 0.003 & & $33.36 \pm 0.12$ & $47.06 \pm 2.60$ & $69.03 \pm 4.03$ \\
\hline & & $g$ & $\mathrm{TF}_{g}$ & 24 & 33.42 & 0.005 & & $33.43 \pm 0.11$ & $48.42 \pm 2.45$ & $67.10 \pm 3.63$ \\
\hline & & $g$ & $\mathrm{TF}_{\mathrm{gc} 1}^{\circ}$ & 24 & 33.35 & 0.005 & & $33.36 \pm 0.11$ & $46.89 \pm 2.38$ & $69.29 \pm 3.74$ \\
\hline & & $g$ & $\mathrm{TF}_{\mathrm{gc} 2}$ & 22 & 33.36 & 0.003 & & $33.36 \pm 0.12$ & $47.06 \pm 2.60$ & $69.04 \pm 4.03$ \\
\hline & & $r$ & $\mathrm{TF}_{r}$ & 24 & 33.33 & 0.003 & & $33.33 \pm 0.11$ & $46.42 \pm 2.35$ & $69.99 \pm 3.78$ \\
\hline & & $r$ & $\mathrm{TF}_{r c}$ & 22 & 33.32 & -0.001 & & $33.32 \pm 0.12$ & $46.12 \pm 2.55$ & $70.45 \pm 4.11$ \\
\hline & & $r$ & $\mathrm{TF}_{r \mu}$ & 24 & 33.31 & 0.000 & & $33.31 \pm 0.11$ & $45.93 \pm 2.33$ & $70.74 \pm 3.82$ \\
\hline & & $i$ & $\mathrm{TF}_{i}$ & 24 & 33.24 & 0.004 & & $33.24 \pm 0.11$ & $44.54 \pm 2.26$ & $72.94 \pm 3.94$ \\
\hline & & $i$ & $\mathrm{TF}_{i c}$ & 22 & 33.24 & 0.005 & & $33.24 \pm 0.12$ & $44.56 \pm 2.46$ & $72.91 \pm 4.26$ \\
\hline & & $i$ & $\mathrm{TF}_{i \mu}$ & 24 & 33.22 & 0.003 & & $33.22 \pm 0.11$ & $44.12 \pm 2.24$ & $73.64 \pm 3.98$ \\
\hline
\end{tabular}


Table 10

(Continued)

\begin{tabular}{|c|c|c|c|c|c|c|c|c|c|c|}
\hline Cluster & $\begin{array}{c}V_{\text {mod }} \\
\mathrm{km} \mathrm{s}^{-1} \\
(2)\end{array}$ & $\begin{array}{c}\text { TFR } \\
\text { Band } \\
\text { (3) }\end{array}$ & $\begin{array}{l}\text { TFR } \\
\text { Model } \\
(4)\end{array}$ & Ngal & $\begin{array}{c}\mathrm{DM}_{o} \\
\operatorname{mag} \\
(6)\end{array}$ & $\begin{array}{l}\text { Bias } \\
\text { mag } \\
(7)\end{array}$ & $\begin{array}{c}\Delta \mathrm{DM} \\
\mathrm{mag} \\
(8)\end{array}$ & $\begin{array}{c}\mathrm{DM}_{c} \\
\mathrm{mag} \\
(9)\end{array}$ & $\begin{array}{c}D \\
\mathrm{Mpc} \\
(10)\end{array}$ & $\begin{array}{c}V_{\mathrm{mod}} / D \\
\mathrm{~km} \mathrm{~s}^{-1} \mathrm{Mpc}^{-1} \\
(11)\end{array}$ \\
\hline & & $z$ & $\mathrm{TF}_{z}$ & 24 & 33.31 & 0.005 & & $33.31 \pm 0.11$ & $46.02 \pm 2.33$ & $70.59 \pm 3.81$ \\
\hline & & $z$ & $\mathrm{TF}_{z c}$ & 22 & 33.30 & 0.002 & & $33.30 \pm 0.11$ & $45.75 \pm 2.32$ & $71.02 \pm 3.84$ \\
\hline & & $z$ & $\mathrm{TF}_{z \mu}$ & 24 & 33.29 & -0.003 & & $33.29 \pm 0.11$ & $45.44 \pm 2.30$ & $71.50 \pm 3.86$ \\
\hline & & $W 1$ & $\mathrm{TF}_{W 1}$ & 23 & 33.17 & 0.001 & & $33.17 \pm 0.12$ & $43.06 \pm 2.38$ & $75.45 \pm 4.40$ \\
\hline & & $W 1$ & $\mathrm{TF}_{W 1 c}$ & 22 & 33.21 & 0.001 & 0.04 & $33.25 \pm 0.12$ & $44.69 \pm 2.47$ & $72.69 \pm 4.24$ \\
\hline & & $W 1$ & $\mathrm{TF}_{W 1 \mu}$ & 23 & 33.05 & -0.000 & 0.11 & $33.16 \pm 0.12$ & $42.85 \pm 2.37$ & $75.82 \pm 4.43$ \\
\hline & & $W 2$ & $\mathrm{TF}_{W 2}$ & 23 & 33.18 & 0.001 & & $33.18 \pm 0.12$ & $43.27 \pm 2.39$ & $75.09 \pm 4.38$ \\
\hline & & $W 2$ & $\mathrm{TF}_{W 2 c}$ & 22 & 33.21 & 0.000 & 0.06 & $33.27 \pm 0.12$ & $45.09 \pm 2.49$ & $72.06 \pm 4.21$ \\
\hline & & $W 2$ & $\mathrm{TF}_{W 2 \mu}$ & 23 & 33.02 & 0.000 & 0.17 & $33.19 \pm 0.11$ & $43.46 \pm 2.20$ & $74.76 \pm 4.04$ \\
\hline \multirow[t]{4}{*}{ Centaurus } & $3645 \pm 56$ & $W 1$ & $\mathrm{TF}_{W 1}$ & 22 & 32.63 & 0.012 & & $32.64 \pm 0.13$ & $33.76 \pm 2.02$ & $107.96 \pm 6.67$ \\
\hline & & $W 1$ & $\mathrm{TF}_{W 1 \mu}$ & 22 & 32.62 & -0.013 & 0.11 & $32.72 \pm 0.13$ & $34.94 \pm 2.09$ & $104.33 \pm 6.45$ \\
\hline & & $W 2$ & $\mathrm{TF}_{W 2}$ & 22 & 32.60 & 0.013 & & $32.61 \pm 0.14$ & $33.31 \pm 2.15$ & $109.44 \pm 7.25$ \\
\hline & & $W 2$ & $\mathrm{TF}_{W 2 \mu}$ & 22 & 32.58 & -0.024 & 0.17 & $32.73 \pm 0.13$ & $35.10 \pm 2.10$ & $103.86 \pm 6.42$ \\
\hline \multirow[t]{4}{*}{ Antlia } & $3103 \pm 53$ & $W 1$ & $\mathrm{TF}_{W 1}$ & 17 & 32.78 & 0.009 & & $32.79 \pm 0.11$ & $36.12 \pm 1.83$ & $85.90 \pm 4.59$ \\
\hline & & $W 1$ & $\mathrm{TF}_{W 1 \mu}$ & 17 & 32.72 & -0.007 & 0.11 & $32.82 \pm 0.10$ & $36.69 \pm 1.69$ & $84.58 \pm 4.15$ \\
\hline & & $W 2$ & $\mathrm{TF}_{W 2}$ & 17 & 32.74 & 0.006 & & $32.75 \pm 0.12$ & $35.42 \pm 1.96$ & $87.60 \pm 5.07$ \\
\hline & & $W 2$ & $\mathrm{TF}_{W 2 \mu}$ & 17 & 32.67 & -0.013 & 0.17 & $32.83 \pm 0.11$ & $36.77 \pm 1.86$ & $84.39 \pm 4.51$ \\
\hline \multirow[t]{4}{*}{ Hydra } & $4084 \pm 44$ & $W 1$ & $\mathrm{TF}_{W 1}$ & 44 & 33.66 & 0.008 & & $33.67 \pm 0.10$ & $54.14 \pm 2.49$ & $75.43 \pm 3.57$ \\
\hline & & $W 1$ & $\mathrm{TF}_{W 1 \mu}$ & 44 & 33.59 & -0.007 & 0.11 & $33.69 \pm 0.09$ & $54.79 \pm 2.27$ & $74.54 \pm 3.19$ \\
\hline & & $W 2$ & $\mathrm{TF}_{W 2}$ & 44 & 33.67 & 0.004 & & $33.67 \pm 0.10$ & $54.31 \pm 2.50$ & $75.19 \pm 3.56$ \\
\hline & & $W 2$ & $\mathrm{TF}_{W 2 \mu}$ & 44 & 33.56 & -0.010 & 0.17 & $33.72 \pm 0.09$ & $55.47 \pm 2.30$ & $73.63 \pm 3.15$ \\
\hline \multirow[t]{4}{*}{ A262 } & $4684 \pm 50$ & $W 1$ & $\mathrm{TF}_{W 1}$ & 54 & 33.85 & 0.006 & & $33.86 \pm 0.13$ & $59.03 \pm 3.53$ & $79.34 \pm 4.83$ \\
\hline & & $W 1$ & $\mathrm{TF}_{W 1 \mu}$ & 54 & 33.81 & -0.005 & 0.11 & $33.91 \pm 0.11$ & $60.67 \pm 3.07$ & $77.21 \pm 4.00$ \\
\hline & & $W 2$ & $\mathrm{TF}_{W 2}$ & 54 & 33.84 & 0.003 & & $33.84 \pm 0.14$ & $58.70 \pm 3.78$ & $79.79 \pm 5.21$ \\
\hline & & $W 2$ & $\mathrm{TF}_{W 2 \mu}$ & 54 & 33.78 & -0.009 & 0.17 & $33.94 \pm 0.12$ & $61.41 \pm 3.39$ & $76.27 \pm 4.29$ \\
\hline \multirow[t]{21}{*}{ NGC 507} & $4660 \pm 64$ & $u$ & $\mathrm{TF}_{u}$ & 20 & 34.07 & 0.027 & & $34.10 \pm 0.15$ & $65.98 \pm 4.56$ & $70.63 \pm 4.97$ \\
\hline & & $u$ & $\mathrm{TF}_{\mathrm{uc} 1}$ & 20 & 33.97 & 0.014 & & $33.98 \pm 0.12$ & $62.64 \pm 3.46$ & $74.40 \pm 4.24$ \\
\hline & & $u$ & $\mathrm{TF}_{\mathrm{uc} 2}$ & 19 & 34.06 & 0.018 & & $34.08 \pm 0.11$ & $65.41 \pm 3.31$ & $71.25 \pm 3.74$ \\
\hline & & $g$ & $\mathrm{TF}_{g}$ & 20 & 34.03 & 0.014 & & $34.04 \pm 0.11$ & $64.40 \pm 3.26$ & $72.36 \pm 3.80$ \\
\hline & & $g$ & $\mathrm{TF}_{\mathrm{gcl}}$ & 20 & 33.99 & 0.019 & & $34.01 \pm 0.12$ & $63.37 \pm 3.50$ & $73.54 \pm 4.19$ \\
\hline & & $g$ & $\mathrm{TF}_{\mathrm{gc} 2}$ & 19 & 34.07 & 0.019 & & $34.09 \pm 0.10$ & $65.72 \pm 3.03$ & $70.90 \pm 3.41$ \\
\hline & & $r$ & $\mathrm{TF}_{r}$ & 20 & 33.98 & 0.014 & & $33.99 \pm 0.11$ & $62.93 \pm 3.19$ & $74.05 \pm 3.89$ \\
\hline & & $r$ & $\mathrm{TF}_{r c}$ & 19 & 34.02 & 0.003 & & $34.02 \pm 0.10$ & $63.75 \pm 2.94$ & $73.09 \pm 3.51$ \\
\hline & & $r$ & $\mathrm{TF}_{r \mu}$ & 20 & 33.95 & 0.005 & & $33.95 \pm 0.11$ & $61.80 \pm 3.13$ & $75.41 \pm 3.96$ \\
\hline & & $i$ & $\mathrm{TF}_{i}$ & 20 & 33.90 & 0.017 & & $33.92 \pm 0.12$ & $60.73 \pm 3.36$ & $76.73 \pm 4.37$ \\
\hline & & $i$ & $\mathrm{TF}_{i c}$ & 19 & 33.97 & 0.021 & & $33.99 \pm 0.09$ & $62.84 \pm 2.60$ & $74.15 \pm 3.24$ \\
\hline & & $i$ & $\mathrm{TF}_{i \mu}$ & 20 & 33.88 & 0.017 & & $33.90 \pm 0.12$ & $60.17 \pm 3.32$ & $77.45 \pm 4.41$ \\
\hline & & $z$ & $\mathrm{TF}_{z}$ & 20 & 33.93 & 0.018 & & $33.95 \pm 0.10$ & $61.60 \pm 2.84$ & $75.65 \pm 3.64$ \\
\hline & & $z$ & $\mathrm{TF}_{z c}$ & 19 & 33.97 & 0.005 & & $33.97 \pm 0.09$ & $62.37 \pm 2.59$ & $74.71 \pm 3.26$ \\
\hline & & $z$ & $\mathrm{TF}_{z \mu}$ & 20 & 33.90 & -0.003 & & $33.90 \pm 0.10$ & $60.17 \pm 2.77$ & $77.45 \pm 3.72$ \\
\hline & & $W 1$ & $\mathrm{TF}_{W 1}$ & 22 & 33.99 & 0.012 & & $34.00 \pm 0.08$ & $63.15 \pm 2.33$ & $73.79 \pm 2.90$ \\
\hline & & $W 1$ & $\mathrm{TF}_{W 1 c}$ & 19 & 33.93 & 0.025 & 0.04 & $33.99 \pm 0.09$ & $62.94 \pm 2.61$ & $74.04 \pm 3.23$ \\
\hline & & $W 1$ & $\mathrm{TF}_{W 1 \mu}$ & 22 & 33.84 & -0.015 & 0.11 & $33.94 \pm 0.07$ & $61.25 \pm 1.97$ & $76.08 \pm 2.67$ \\
\hline & & $W 2$ & $\mathrm{TF}_{W 2}$ & 22 & 34.04 & 0.013 & & $34.05 \pm 0.09$ & $64.65 \pm 2.68$ & $72.08 \pm 3.15$ \\
\hline & & $W 2$ & $\mathrm{TF}_{W 2 c}$ & 19 & 33.92 & 0.004 & 0.06 & $33.98 \pm 0.09$ & $62.65 \pm 2.60$ & $74.39 \pm 3.25$ \\
\hline & & $W 2$ & $\mathrm{TF}_{W 2 \mu}$ & 22 & 33.84 & -0.023 & 0.17 & $33.99 \pm 0.08$ & $62.73 \pm 2.31$ & $74.28 \pm 2.92$ \\
\hline \multirow[t]{9}{*}{ NGC 410} & $4792 \pm 53$ & $u$ & $\mathrm{TF}_{u}$ & 33 & 34.09 & 0.008 & & $34.10 \pm 0.09$ & $66.02 \pm 2.74$ & $72.58 \pm 3.11$ \\
\hline & & $u$ & $\mathrm{TF}_{\mathrm{uc} 1}$ & 33 & 34.05 & 0.006 & & $34.06 \pm 0.08$ & $64.74 \pm 2.39$ & $74.02 \pm 2.85$ \\
\hline & & $u$ & $\mathrm{TF}_{\mathrm{uc} 2}$ & 31 & 34.05 & 0.006 & & $34.06 \pm 0.08$ & $64.76 \pm 2.39$ & $74.00 \pm 2.85$ \\
\hline & & $g$ & $\mathrm{TF}_{g}$ & 33 & 34.07 & 0.010 & & $34.08 \pm 0.07$ & $65.45 \pm 2.11$ & $73.21 \pm 2.50$ \\
\hline & & $g$ & $\mathrm{TF}_{\mathrm{gc} 1}$ & 33 & 34.06 & 0.013 & & $34.07 \pm 0.08$ & $65.26 \pm 2.40$ & $73.43 \pm 2.82$ \\
\hline & & $g$ & $\mathrm{TF}_{\mathrm{gc} 2}$ & 31 & 34.06 & 0.010 & & $34.07 \pm 0.08$ & $65.16 \pm 2.40$ & $73.54 \pm 2.83$ \\
\hline & & $r$ & $\mathrm{TF}_{r}$ & 33 & 34.03 & 0.007 & & $34.04 \pm 0.08$ & $64.19 \pm 2.36$ & $74.65 \pm 2.87$ \\
\hline & & $r$ & $\mathrm{TF}_{r c}$ & 31 & 34.04 & -0.000 & & $34.04 \pm 0.08$ & $64.26 \pm 2.37$ & $74.57 \pm 2.87$ \\
\hline & & $r$ & $\mathrm{TF}_{r \mu}$ & 33 & 34.02 & 0.002 & & $34.02 \pm 0.07$ & $63.73 \pm 2.05$ & $75.19 \pm 2.56$ \\
\hline
\end{tabular}


Table 10

(Continued)

\begin{tabular}{|c|c|c|c|c|c|c|c|c|c|c|}
\hline Cluster & $\begin{array}{c}V_{\text {mod }} \\
\mathrm{km} \mathrm{s}^{-1} \\
(2)\end{array}$ & $\begin{array}{c}\text { TFR } \\
\text { Band } \\
(3)\end{array}$ & $\begin{array}{l}\text { TFR } \\
\text { Model } \\
(4)\end{array}$ & Ngal & $\begin{array}{c}\mathrm{DM}_{o} \\
\operatorname{mag} \\
(6)\end{array}$ & $\begin{array}{c}\text { Bias } \\
\text { mag } \\
(7)\end{array}$ & $\begin{array}{c}\Delta \mathrm{DM} \\
\mathrm{mag} \\
(8)\end{array}$ & $\begin{array}{c}\mathrm{DM}_{c} \\
\mathrm{mag} \\
(9)\end{array}$ & $\begin{array}{c}D \\
\mathrm{Mpc} \\
(10)\end{array}$ & $\begin{array}{c}V_{\mathrm{mod}} / D \\
\mathrm{~km} \mathrm{~s}^{-1} \mathrm{Mpc}^{-1} \\
(11)\end{array}$ \\
\hline & & $i$ & $\mathrm{TF}_{i}$ & 33 & 33.99 & 0.006 & & $34.00 \pm 0.08$ & $62.98 \pm 2.32$ & $76.08 \pm 2.93$ \\
\hline & & $i$ & $\mathrm{TF}_{i c}$ & 31 & 34.00 & 0.007 & & $34.01 \pm 0.08$ & $63.31 \pm 2.33$ & $75.69 \pm 2.91$ \\
\hline & & $i$ & $\mathrm{TF}_{i \mu}$ & 33 & 33.97 & 0.005 & & $33.98 \pm 0.08$ & $62.38 \pm 2.30$ & $76.82 \pm 2.95$ \\
\hline & & $z$ & $\mathrm{TF}_{z}$ & 33 & 33.99 & 0.009 & & $34.00 \pm 0.08$ & $63.08 \pm 2.32$ & $75.97 \pm 2.92$ \\
\hline & & $z$ & $\mathrm{TF}_{z c}$ & 31 & 34.01 & 0.003 & & $34.01 \pm 0.07$ & $63.48 \pm 2.05$ & $75.49 \pm 2.57$ \\
\hline & & $z$ & $\mathrm{TF}_{z \mu}$ & 33 & 33.98 & -0.004 & & $33.98 \pm 0.08$ & $62.41 \pm 2.30$ & $76.79 \pm 2.95$ \\
\hline & & $W 1$ & $\mathrm{TF}_{W 1}$ & 31 & 33.96 & 0.004 & & $33.96 \pm 0.10$ & $62.05 \pm 2.86$ & $77.23 \pm 3.66$ \\
\hline & & $W 1$ & $\mathrm{TF}_{W 1 c}$ & 31 & 33.96 & 0.005 & 0.04 & $34.00 \pm 0.08$ & $63.24 \pm 2.33$ & $75.78 \pm 2.91$ \\
\hline & & $W 1$ & $\mathrm{TF}_{W 1 \mu}$ & 31 & 33.85 & -0.004 & 0.11 & $33.96 \pm 0.09$ & $61.84 \pm 2.56$ & $77.49 \pm 3.32$ \\
\hline & & $W 2$ & $\mathrm{TF}_{W 2}$ & 31 & 33.96 & 0.003 & & $33.96 \pm 0.10$ & $62.02 \pm 2.86$ & $77.27 \pm 3.66$ \\
\hline & & $W 2$ & $\mathrm{TF}_{W 2 c}$ & 31 & 33.96 & 0.001 & 0.06 & $34.02 \pm 0.08$ & $63.71 \pm 2.35$ & $75.22 \pm 2.89$ \\
\hline & & $W 2$ & $\mathrm{TF}_{W 2 \mu}$ & 31 & 33.82 & -0.007 & 0.17 & $33.98 \pm 0.09$ & $62.61 \pm 2.60$ & $76.54 \pm 3.28$ \\
\hline \multirow[t]{21}{*}{ Cancer } & $5025 \pm 71$ & $u$ & $\mathrm{TF}_{u}$ & 18 & 34.24 & 0.071 & & $34.31 \pm 0.14$ & $72.80 \pm 4.69$ & $69.02 \pm 4.56$ \\
\hline & & $u$ & $\mathrm{TF}_{\mathrm{uc} 1}$ & 18 & 34.19 & 0.040 & & $34.23 \pm 0.11$ & $70.15 \pm 3.55$ & $71.63 \pm 3.77$ \\
\hline & & $u$ & $\mathrm{TF}_{\mathrm{uc} 2}$ & 17 & 34.22 & 0.045 & & $34.27 \pm 0.11$ & $71.30 \pm 3.61$ & $70.48 \pm 3.71$ \\
\hline & & $g$ & $\mathrm{TF}_{g}$ & 18 & 34.19 & 0.035 & & $34.23 \pm 0.10$ & $69.99 \pm 3.22$ & $71.80 \pm 3.46$ \\
\hline & & $g$ & $\mathrm{TF}_{\mathrm{gcl}}^{\circ}$ & 18 & 34.20 & 0.057 & & $34.26 \pm 0.11$ & $71.02 \pm 3.60$ & $70.76 \pm 3.72$ \\
\hline & & $g$ & $\mathrm{TF}_{\mathrm{gc} 2}$ & 17 & 34.21 & 0.056 & & $34.27 \pm 0.10$ & $71.31 \pm 3.28$ & $70.46 \pm 3.39$ \\
\hline & & $r$ & $\mathrm{TF}_{r}$ & 18 & 34.18 & 0.032 & & $34.21 \pm 0.11$ & $69.56 \pm 3.52$ & $72.24 \pm 3.80$ \\
\hline & & $r$ & $\mathrm{TF}_{r c}$ & 17 & 34.19 & 0.012 & & $34.20 \pm 0.11$ & $69.25 \pm 3.51$ & $72.56 \pm 3.82$ \\
\hline & & $r$ & $\mathrm{TF}_{r \mu}$ & 18 & 34.15 & 0.019 & & $34.17 \pm 0.11$ & $68.21 \pm 3.46$ & $73.67 \pm 3.87$ \\
\hline & & $i$ & $\mathrm{TF}_{i}$ & 18 & 34.14 & 0.030 & & $34.17 \pm 0.11$ & $68.24 \pm 3.46$ & $73.64 \pm 3.87$ \\
\hline & & $i$ & $\mathrm{TF}_{i c}$ & 17 & 34.16 & 0.038 & & $34.20 \pm 0.11$ & $69.13 \pm 3.50$ & $72.69 \pm 3.82$ \\
\hline & & $i$ & $\mathrm{TF}_{i \mu}$ & 18 & 34.11 & 0.032 & & $34.14 \pm 0.11$ & $67.35 \pm 3.41$ & $74.61 \pm 3.92$ \\
\hline & & $z$ & $\mathrm{TF}_{z}$ & 18 & 34.20 & 0.027 & & $34.23 \pm 0.11$ & $70.04 \pm 3.55$ & $71.74 \pm 3.77$ \\
\hline & & $z$ & $\mathrm{TF}_{z c}$ & 17 & 34.22 & 0.007 & & $34.23 \pm 0.12$ & $70.05 \pm 3.87$ & $71.73 \pm 4.09$ \\
\hline & & $z$ & $\mathrm{TF}_{z \mu}$ & 18 & 34.17 & -0.003 & & $34.17 \pm 0.11$ & $68.14 \pm 3.45$ & $73.74 \pm 3.88$ \\
\hline & & $W 1$ & $\mathrm{TF}_{W 1}$ & 17 & 34.15 & 0.012 & & $34.16 \pm 0.11$ & $67.98 \pm 3.44$ & $73.92 \pm 3.89$ \\
\hline & & $W 1$ & $\mathrm{TF}_{W 1 c}$ & 17 & 34.12 & 0.026 & 0.04 & $34.19 \pm 0.11$ & $68.73 \pm 3.48$ & $73.11 \pm 3.85$ \\
\hline & & $W 1$ & $\mathrm{TF}_{W 1 \mu}$ & 17 & 34.03 & -0.015 & 0.11 & $34.12 \pm 0.10$ & $66.82 \pm 3.08$ & $75.20 \pm 3.62$ \\
\hline & & $W 2$ & $\mathrm{TF}_{W 2}$ & 17 & 34.14 & 0.013 & & $34.15 \pm 0.11$ & $67.70 \pm 3.43$ & $74.22 \pm 3.90$ \\
\hline & & $W 2$ & $\mathrm{TF}_{W 2 c}$ & 17 & 34.12 & 0.006 & 0.06 & $34.19 \pm 0.11$ & $68.73 \pm 3.48$ & $73.12 \pm 3.85$ \\
\hline & & $W 2$ & $\mathrm{TF}_{W 2 \mu}$ & 17 & 33.98 & -0.027 & 0.17 & $34.12 \pm 0.11$ & $66.76 \pm 3.38$ & $75.26 \pm 3.96$ \\
\hline \multirow[t]{21}{*}{ NGC 80} & $5499 \pm 42$ & $u$ & $\mathrm{TF}_{u}$ & 14 & 34.72 & 0.046 & & $34.77 \pm 0.10$ & $89.77 \pm 4.13$ & $61.25 \pm 2.86$ \\
\hline & & $u$ & $\mathrm{TF}_{\mathrm{uc} 1}$ & 14 & 34.61 & 0.024 & & $34.63 \pm 0.07$ & $84.47 \pm 2.72$ & $65.10 \pm 2.16$ \\
\hline & & $u$ & $\mathrm{TF}_{\mathrm{uc} 2}$ & 13 & 34.61 & 0.026 & & $34.64 \pm 0.09$ & $84.57 \pm 3.51$ & $65.02 \pm 2.74$ \\
\hline & & $g$ & $\mathrm{TF}_{g}$ & 14 & 34.67 & 0.024 & & $34.69 \pm 0.08$ & $86.87 \pm 3.20$ & $63.30 \pm 2.38$ \\
\hline & & $g$ & $\mathrm{TF}_{\mathrm{gcl} 1}^{8}$ & 14 & 34.62 & 0.035 & & $34.66 \pm 0.07$ & $85.32 \pm 2.75$ & $64.45 \pm 2.14$ \\
\hline & & $g$ & $\mathrm{TF}_{\mathrm{gc} 2}$ & 13 & 34.62 & 0.032 & & $34.65 \pm 0.08$ & $85.20 \pm 3.14$ & $64.54 \pm 2.43$ \\
\hline & & $r$ & $\mathrm{TF}_{r}$ & 14 & 34.59 & 0.023 & & $34.61 \pm 0.08$ & $83.67 \pm 3.08$ & $65.72 \pm 2.47$ \\
\hline & & $r$ & $\mathrm{TF}_{r c}$ & 13 & 34.60 & 0.006 & & $34.61 \pm 0.08$ & $83.42 \pm 3.07$ & $65.92 \pm 2.48$ \\
\hline & & $r$ & $\mathrm{TF}_{r \mu}$ & 14 & 34.60 & 0.010 & & $34.61 \pm 0.07$ & $83.58 \pm 2.69$ & $65.80 \pm 2.18$ \\
\hline & & $i$ & $\mathrm{TF}_{i}$ & 14 & 34.53 & 0.023 & & $34.55 \pm 0.07$ & $81.39 \pm 2.62$ & $67.57 \pm 2.24$ \\
\hline & & $i$ & $\mathrm{TF}_{i c}$ & 13 & 34.55 & 0.027 & & $34.58 \pm 0.07$ & $82.29 \pm 2.65$ & $66.82 \pm 2.21$ \\
\hline & & $i$ & $\mathrm{TF}_{i \mu}$ & 14 & 34.55 & 0.024 & & $34.57 \pm 0.07$ & $82.17 \pm 2.65$ & $66.92 \pm 2.22$ \\
\hline & & $z$ & $\mathrm{TF}_{z}$ & 14 & 34.53 & 0.022 & & $34.55 \pm 0.07$ & $81.36 \pm 2.62$ & $67.59 \pm 2.24$ \\
\hline & & $z$ & $\mathrm{TF}_{z c}$ & 13 & 34.55 & 0.006 & & $34.56 \pm 0.07$ & $81.50 \pm 2.63$ & $67.47 \pm 2.24$ \\
\hline & & $z$ & $\mathrm{TF}_{z \mu}$ & 14 & 34.55 & -0.002 & & $34.55 \pm 0.06$ & $81.20 \pm 2.24$ & $67.72 \pm 1.94$ \\
\hline & & $W 1$ & $\mathrm{TF}_{W 1}$ & 13 & 34.45 & 0.011 & & $34.46 \pm 0.12$ & $78.02 \pm 4.31$ & $70.49 \pm 3.93$ \\
\hline & & $W 1$ & $\mathrm{TF}_{W 1 c}$ & 13 & 34.50 & 0.029 & 0.04 & $34.57 \pm 0.07$ & $82.01 \pm 2.64$ & $67.06 \pm 2.22$ \\
\hline & & $W 1$ & $\mathrm{TF}_{W 1 \mu}$ & 13 & 34.45 & -0.019 & 0.11 & $34.54 \pm 0.09$ & $80.93 \pm 3.35$ & $67.94 \pm 2.86$ \\
\hline & & $W 2$ & $\mathrm{TF}_{W 2}$ & 13 & 34.37 & 0.012 & & $34.38 \pm 0.16$ & $75.25 \pm 5.54$ & $73.08 \pm 5.41$ \\
\hline & & $W 2$ & $\mathrm{TF}_{W 2 c}$ & 13 & 34.51 & 0.007 & 0.06 & $34.58 \pm 0.07$ & $82.29 \pm 2.65$ & $66.82 \pm 2.21$ \\
\hline & & $W 2$ & $\mathrm{TF}_{W 2 \mu}$ & 13 & 34.35 & -0.033 & 0.17 & $34.49 \pm 0.13$ & $78.95 \pm 4.73$ & $69.66 \pm 4.20$ \\
\hline \multirow[t]{4}{*}{ NGC 70} & $6619 \pm 80$ & $u$ & $\mathrm{TF}_{u}$ & 11 & 34.74 & 0.085 & & $34.83 \pm 0.14$ & $92.28 \pm 5.95$ & $71.73 \pm 4.71$ \\
\hline & & $u$ & $\mathrm{TF}_{\mathrm{ucl} 1}$ & 11 & 34.62 & 0.051 & & $34.67 \pm 0.11$ & $85.96 \pm 4.35$ & $77.00 \pm 4.01$ \\
\hline & & $u$ & $\mathrm{TF}_{\mathrm{uc} 2}$ & 11 & 34.63 & 0.057 & & $34.69 \pm 0.12$ & $86.56 \pm 4.78$ & $76.47 \pm 4.33$ \\
\hline & & $g$ & $\mathrm{TF}_{g}$ & 11 & 34.66 & 0.036 & & $34.70 \pm 0.11$ & $86.93 \pm 4.40$ & $76.14 \pm 3.97$ \\
\hline
\end{tabular}


Table 10

(Continued)

\begin{tabular}{|c|c|c|c|c|c|c|c|c|c|c|}
\hline Cluster & $\begin{array}{c}V_{\mathrm{mod}} \\
\mathrm{km} \mathrm{s}^{-1} \\
(2)\end{array}$ & $\begin{array}{l}\text { TFR } \\
\text { Band } \\
(3)\end{array}$ & $\begin{array}{l}\text { TFR } \\
\text { Model } \\
\text { (4) }\end{array}$ & Ngal & $\begin{array}{c}\mathrm{DM}_{o} \\
\mathrm{mag} \\
(6)\end{array}$ & $\begin{array}{l}\text { Bias } \\
\text { mag } \\
(7)\end{array}$ & $\begin{array}{c}\Delta \mathrm{DM} \\
\mathrm{mag} \\
(8)\end{array}$ & $\begin{array}{c}\mathrm{DM}_{c} \\
\text { mag } \\
(9)\end{array}$ & $\begin{array}{c}D \\
\mathrm{Mpc} \\
(10)\end{array}$ & $\begin{array}{c}V_{\text {mod }} / D \\
\mathrm{~km} \mathrm{~s}^{-1} \mathrm{Mpc}^{-1} \\
\text { (11) }\end{array}$ \\
\hline & & $g$ & $\mathrm{TF}_{\mathrm{gc} 1}$ & 11 & 34.64 & 0.059 & & $34.70 \pm 0.11$ & $87.07 \pm 4.41$ & $76.02 \pm 3.96$ \\
\hline & & $g$ & $\mathrm{TF}_{\mathrm{gc} 2}$ & 11 & 34.63 & 0.059 & & $34.69 \pm 0.11$ & $86.64 \pm 4.39$ & $76.40 \pm 3.98$ \\
\hline & & $r$ & $\mathrm{TF}_{r}$ & 11 & 34.62 & 0.030 & & $34.65 \pm 0.10$ & $85.10 \pm 3.92$ & $77.77 \pm 3.70$ \\
\hline & & $r$ & $\mathrm{TF}_{r c}$ & 11 & 34.62 & 0.012 & & $34.63 \pm 0.10$ & $84.42 \pm 3.89$ & $78.41 \pm 3.73$ \\
\hline & & $r$ & $\mathrm{TF}_{r \mu}$ & 11 & 34.62 & 0.022 & & $34.64 \pm 0.11$ & $84.80 \pm 4.30$ & $78.05 \pm 4.06$ \\
\hline & & $i$ & $\mathrm{TF}_{i}$ & 11 & 34.56 & 0.028 & & $34.59 \pm 0.10$ & $82.73 \pm 3.81$ & $80.01 \pm 3.81$ \\
\hline & & $i$ & $\mathrm{TF}_{i c}$ & 11 & 34.56 & 0.040 & & $34.60 \pm 0.10$ & $83.18 \pm 3.83$ & $79.57 \pm 3.79$ \\
\hline & & $i$ & $\mathrm{TF}_{i \mu}$ & 11 & 34.56 & 0.031 & & $34.59 \pm 0.11$ & $82.82 \pm 4.20$ & $79.92 \pm 4.16$ \\
\hline & & $z$ & $\mathrm{TF}_{z}$ & 11 & 34.56 & 0.022 & & $34.58 \pm 0.10$ & $82.49 \pm 3.80$ & $80.24 \pm 3.82$ \\
\hline & & $z$ & $\mathrm{TF}_{z c}$ & 11 & 34.56 & 0.007 & & $34.57 \pm 0.10$ & $81.92 \pm 3.77$ & $80.80 \pm 3.85$ \\
\hline & & $z$ & $\mathrm{TF}_{z \mu}$ & 11 & 34.57 & -0.010 & & $34.56 \pm 0.11$ & $81.67 \pm 4.14$ & $81.04 \pm 4.22$ \\
\hline & & $W 1$ & $\mathrm{TF}_{W 1}$ & 11 & 34.52 & 0.002 & & $34.52 \pm 0.12$ & $80.23 \pm 4.43$ & $82.50 \pm 4.67$ \\
\hline & & $W 1$ & $\mathrm{TF}_{W 1 c}$ & 11 & 34.50 & 0.037 & 0.04 & $34.58 \pm 0.10$ & $82.31 \pm 3.79$ & $80.42 \pm 3.83$ \\
\hline & & $W 1$ & $\mathrm{TF}_{W 1 \mu}$ & 11 & 34.48 & -0.031 & 0.11 & $34.56 \pm 0.12$ & $81.61 \pm 4.51$ & $81.11 \pm 4.59$ \\
\hline & & $W 2$ & $\mathrm{TF}_{W 2}$ & 11 & 34.51 & 0.004 & & $34.51 \pm 0.12$ & $79.96 \pm 4.42$ & $82.78 \pm 4.68$ \\
\hline & & $W 2$ & $\mathrm{TF}_{W 2 c}$ & 11 & 34.50 & 0.009 & 0.06 & $34.57 \pm 0.10$ & $81.98 \pm 3.78$ & $80.74 \pm 3.84$ \\
\hline & & $W 2$ & $\mathrm{TF}_{W 2 \mu}$ & 11 & 34.45 & -0.051 & 0.17 & $34.57 \pm 0.13$ & $82.01 \pm 4.91$ & $80.71 \pm 4.93$ \\
\hline \multirow[t]{21}{*}{ A 1367} & $7060 \pm 61$ & $u$ & $\mathrm{TF}_{u}$ & 68 & 34.67 & 0.008 & & $34.68 \pm 0.07$ & $86.20 \pm 2.78$ & $81.90 \pm 2.73$ \\
\hline & & $u$ & $\mathrm{TF}_{\mathrm{uc} 1}$ & 68 & 34.77 & 0.008 & & $34.78 \pm 0.06$ & $90.28 \pm 2.49$ & $78.21 \pm 2.26$ \\
\hline & & $u$ & $\mathrm{TF}_{\mathrm{uc} 2}$ & 62 & 34.78 & 0.009 & & $34.79 \pm 0.06$ & $90.74 \pm 2.51$ & $77.81 \pm 2.25$ \\
\hline & & $g$ & $\mathrm{TF}_{g}$ & 68 & 34.75 & 0.012 & & $34.76 \pm 0.06$ & $89.61 \pm 2.48$ & $78.78 \pm 2.28$ \\
\hline & & $g$ & $\mathrm{TF}_{\mathrm{gcl}}$ & 68 & 34.81 & 0.019 & & $34.83 \pm 0.06$ & $92.41 \pm 2.55$ & $76.40 \pm 2.21$ \\
\hline & & $g$ & $\mathrm{TF}_{\mathrm{gc} 2}$ & 62 & 34.81 & 0.015 & & $34.83 \pm 0.06$ & $92.26 \pm 2.55$ & $76.52 \pm 2.22$ \\
\hline & & $r$ & $\mathrm{TF}_{r}$ & 68 & 34.76 & 0.010 & & $34.77 \pm 0.06$ & $89.97 \pm 2.49$ & $78.47 \pm 2.27$ \\
\hline & & $r$ & $\mathrm{TF}_{r c}$ & 62 & 34.76 & 0.001 & & $34.76 \pm 0.06$ & $89.57 \pm 2.47$ & $78.82 \pm 2.28$ \\
\hline & & $r$ & $\mathrm{TF}_{r \mu}$ & 68 & 34.75 & 0.003 & & $34.75 \pm 0.06$ & $89.25 \pm 2.47$ & $79.10 \pm 2.29$ \\
\hline & & $i$ & $\mathrm{TF}_{i}$ & 68 & 34.76 & 0.013 & & $34.77 \pm 0.06$ & $90.08 \pm 2.49$ & $78.37 \pm 2.27$ \\
\hline & & $i$ & $\mathrm{TF}_{i c}$ & 62 & 34.76 & 0.015 & & $34.78 \pm 0.06$ & $90.18 \pm 2.49$ & $78.29 \pm 2.27$ \\
\hline & & $i$ & $\mathrm{TF}_{i \mu}$ & 68 & 34.74 & 0.013 & & $34.75 \pm 0.06$ & $89.23 \pm 2.47$ & $79.12 \pm 2.29$ \\
\hline & & $z$ & $\mathrm{TF}_{z}$ & 68 & 34.80 & 0.011 & & $34.81 \pm 0.06$ & $91.64 \pm 2.53$ & $77.04 \pm 2.23$ \\
\hline & & $z$ & $\mathrm{TF}_{z c}$ & 62 & 34.78 & 0.003 & & $34.78 \pm 0.06$ & $90.50 \pm 2.50$ & $78.01 \pm 2.26$ \\
\hline & & $z$ & $\mathrm{TF}_{z \mu}$ & 68 & 34.78 & -0.004 & & $34.78 \pm 0.06$ & $90.20 \pm 2.49$ & $78.27 \pm 2.27$ \\
\hline & & $W 1$ & $\mathrm{TF}_{W 1}$ & 62 & 34.85 & 0.011 & & $34.86 \pm 0.06$ & $93.80 \pm 2.59$ & $75.26 \pm 2.18$ \\
\hline & & $W 1$ & $\mathrm{TF}_{W 1 c}$ & 62 & 34.73 & 0.015 & 0.04 & $34.79 \pm 0.06$ & $90.58 \pm 2.50$ & $77.94 \pm 2.26$ \\
\hline & & $W 1$ & $\mathrm{TF}_{W 1 \mu}$ & 62 & 34.73 & -0.009 & 0.11 & $34.83 \pm 0.06$ & $92.50 \pm 2.56$ & $76.32 \pm 2.21$ \\
\hline & & $W 2$ & $\mathrm{TF}_{W 2}$ & 62 & 34.83 & 0.010 & & $34.84 \pm 0.07$ & $92.88 \pm 2.99$ & $76.01 \pm 2.54$ \\
\hline & & $W 2$ & $\mathrm{TF}_{W 2 c}$ & 62 & 34.73 & 0.003 & 0.06 & $34.79 \pm 0.06$ & $90.90 \pm 2.51$ & $77.67 \pm 2.25$ \\
\hline & & $W 2$ & $\mathrm{TF}_{W 2 \mu}$ & 62 & 34.67 & -0.016 & 0.17 & $34.82 \pm 0.06$ & $92.23 \pm 2.55$ & $76.55 \pm 2.22$ \\
\hline \multirow[t]{21}{*}{ Coma } & $7352 \pm 70$ & $u$ & $\mathrm{TF}_{u}$ & 79 & 34.68 & 0.027 & & $34.71 \pm 0.07$ & $87.40 \pm 2.82$ & $84.12 \pm 2.83$ \\
\hline & & $u$ & $\mathrm{TF}_{\mathrm{uc} 1}$ & 79 & 34.80 & 0.020 & & $34.82 \pm 0.06$ & $92.05 \pm 2.54$ & $79.87 \pm 2.33$ \\
\hline & & $u$ & $\mathrm{TF}_{\mathrm{uc} 2}$ & 75 & 34.79 & 0.022 & & $34.81 \pm 0.06$ & $91.70 \pm 2.53$ & $80.17 \pm 2.34$ \\
\hline & & $g$ & $\mathrm{TF}_{g}$ & 79 & 34.78 & 0.012 & & $34.79 \pm 0.06$ & $90.87 \pm 2.51$ & $80.91 \pm 2.36$ \\
\hline & & $g$ & $\mathrm{TF}_{\mathrm{gc} 1}^{\circ}$ & 79 & 34.84 & 0.019 & & $34.86 \pm 0.06$ & $93.70 \pm 2.59$ & $78.46 \pm 2.29$ \\
\hline & & $g$ & $\mathrm{TF}_{\mathrm{gc} 2}$ & 75 & 34.83 & 0.015 & & $34.85 \pm 0.06$ & $93.12 \pm 2.57$ & $78.95 \pm 2.31$ \\
\hline & & $r$ & $\mathrm{TF}_{r}$ & 79 & 34.80 & 0.010 & & $34.81 \pm 0.06$ & $91.64 \pm 2.53$ & $80.23 \pm 2.34$ \\
\hline & & $r$ & $\mathrm{TF}_{r c}$ & 75 & 34.78 & 0.001 & & $34.78 \pm 0.06$ & $90.39 \pm 2.50$ & $81.34 \pm 2.38$ \\
\hline & & $r$ & $\mathrm{TF}_{r \mu}$ & 79 & 34.75 & 0.003 & & $34.75 \pm 0.06$ & $89.24 \pm 2.47$ & $82.39 \pm 2.41$ \\
\hline & & $i$ & $\mathrm{TF}_{i}$ & 79 & 34.79 & 0.009 & & $34.80 \pm 0.06$ & $91.16 \pm 2.52$ & $80.65 \pm 2.36$ \\
\hline & & $i$ & $\mathrm{TF}_{i c}$ & 75 & 34.77 & 0.010 & & $34.78 \pm 0.06$ & $90.38 \pm 2.50$ & $81.34 \pm 2.38$ \\
\hline & & $i$ & $\mathrm{TF}_{i \mu}$ & 79 & 34.74 & 0.008 & & $34.75 \pm 0.06$ & $89.04 \pm 2.46$ & $82.57 \pm 2.41$ \\
\hline & & $z$ & $\mathrm{TF}_{z}$ & 79 & 34.82 & 0.008 & & $34.83 \pm 0.06$ & $92.38 \pm 2.55$ & $79.58 \pm 2.33$ \\
\hline & & $z$ & $\mathrm{TF}_{z c}$ & 75 & 34.80 & 0.003 & & $34.80 \pm 0.06$ & $91.31 \pm 2.52$ & $80.52 \pm 2.35$ \\
\hline & & $z$ & $\mathrm{TF}_{z \mu}$ & 79 & 34.77 & -0.004 & & $34.77 \pm 0.06$ & $89.80 \pm 2.48$ & $81.87 \pm 2.39$ \\
\hline & & $W 1$ & $\mathrm{TF}_{W 1}$ & 75 & 34.85 & 0.004 & & $34.85 \pm 0.06$ & $93.49 \pm 2.58$ & $78.64 \pm 2.30$ \\
\hline & & $W 1$ & $\mathrm{TF}_{W 1 c}$ & 75 & 34.74 & 0.004 & 0.04 & $34.78 \pm 0.06$ & $90.54 \pm 2.50$ & $81.20 \pm 2.37$ \\
\hline & & $W 1$ & $\mathrm{TF}_{W 1 \mu}$ & 75 & 34.69 & -0.004 & 0.11 & $34.80 \pm 0.06$ & $91.05 \pm 2.52$ & $80.74 \pm 2.36$ \\
\hline & & $W 2$ & $\mathrm{TF}_{W 2}$ & 75 & 34.86 & 0.002 & & $34.86 \pm 0.07$ & $93.86 \pm 3.03$ & $78.33 \pm 2.63$ \\
\hline & & $W 2$ & $\mathrm{TF}_{W 2 c}$ & 75 & 34.74 & 0.001 & 0.06 & $34.80 \pm 0.06$ & $91.23 \pm 2.52$ & $80.59 \pm 2.36$ \\
\hline & & $W 2$ & $\mathrm{TF}_{W 2 \mu}$ & 75 & 34.64 & -0.005 & 0.17 & $34.80 \pm 0.06$ & $91.40 \pm 2.53$ & $80.44 \pm 2.35$ \\
\hline
\end{tabular}


Table 10

(Continued)

\begin{tabular}{|c|c|c|c|c|c|c|c|c|c|c|}
\hline $\begin{array}{l}\text { Cluster } \\
\text { (1) }\end{array}$ & $\begin{array}{c}V_{\mathrm{mod}} \\
\mathrm{km} \mathrm{s}_{(2)}\end{array}$ & $\begin{array}{c}\text { TFR } \\
\text { Band } \\
\text { (3) }\end{array}$ & $\begin{array}{l}\text { TFR } \\
\text { Model } \\
(4)\end{array}$ & $\begin{array}{c}\text { Ngal } \\
(5)\end{array}$ & $\begin{array}{c}\mathrm{DM}_{o} \\
\text { mag } \\
(6)\end{array}$ & $\begin{array}{l}\text { Bias } \\
\text { mag } \\
(7)\end{array}$ & $\begin{array}{c}\Delta \mathrm{DM} \\
\mathrm{mag} \\
(8)\end{array}$ & $\begin{array}{c}\mathrm{DM}_{c} \\
\mathrm{mag} \\
(9)\end{array}$ & $\begin{array}{c}D \\
\mathrm{Mpc} \\
(10)\end{array}$ & $\begin{array}{c}V_{\mathrm{mod}} / D \\
\mathrm{~km} \mathrm{~s}^{-1} \mathrm{Mpc}^{-1} \\
(11)\end{array}$ \\
\hline \multirow[t]{21}{*}{ A400 } & \multirow[t]{21}{*}{$7357 \pm 85$} & $u$ & $\mathrm{TF}_{u}$ & 21 & 34.72 & 0.048 & & $34.77 \pm 0.10$ & $89.88 \pm 4.14$ & $81.85 \pm 3.89$ \\
\hline & & $u$ & $\mathrm{TF}_{\mathrm{uc} 1}$ & 21 & 34.84 & 0.032 & & $34.87 \pm 0.06$ & $94.26 \pm 2.60$ & $78.05 \pm 2.34$ \\
\hline & & $u$ & $\mathrm{TF}_{\mathrm{uc} 2}$ & 20 & 34.82 & 0.034 & & $34.85 \pm 0.07$ & $93.51 \pm 3.01$ & $78.68 \pm 2.69$ \\
\hline & & $g$ & $\mathrm{TF}_{g}$ & 21 & 34.82 & 0.031 & & $34.85 \pm 0.07$ & $93.36 \pm 3.01$ & $78.81 \pm 2.70$ \\
\hline & & $g$ & $\mathrm{TF}_{\mathrm{gc} 1}^{\circ}$ & 21 & 34.88 & 0.049 & & $34.93 \pm 0.06$ & $96.80 \pm 2.67$ & $76.00 \pm 2.28$ \\
\hline & & $g$ & $\mathrm{TF}_{\mathrm{gc} 2}$ & 20 & 34.86 & 0.047 & & $34.91 \pm 0.07$ & $95.81 \pm 3.09$ & $76.79 \pm 2.63$ \\
\hline & & $r$ & $\mathrm{TF}_{r}$ & 21 & 34.85 & 0.030 & & $34.88 \pm 0.06$ & $94.63 \pm 2.61$ & $77.74 \pm 2.33$ \\
\hline & & $r$ & $\mathrm{TF}_{r c}$ & 20 & 34.85 & 0.010 & & $34.86 \pm 0.06$ & $93.78 \pm 2.59$ & $78.45 \pm 2.35$ \\
\hline & & $r$ & $\mathrm{TF}_{r \mu}$ & 21 & 34.84 & 0.016 & & $34.86 \pm 0.06$ & $93.60 \pm 2.59$ & $78.60 \pm 2.35$ \\
\hline & & $i$ & $\mathrm{TF}_{i}$ & 21 & 34.84 & 0.029 & & $34.87 \pm 0.06$ & $94.15 \pm 2.60$ & $78.14 \pm 2.34$ \\
\hline & & $i$ & $\mathrm{TF}_{i c}$ & 20 & 34.83 & 0.035 & & $34.86 \pm 0.06$ & $93.97 \pm 2.60$ & $78.29 \pm 2.34$ \\
\hline & & $i$ & $\mathrm{TF}_{i \mu}$ & 21 & 34.82 & 0.030 & & $34.85 \pm 0.06$ & $93.33 \pm 2.58$ & $78.83 \pm 2.36$ \\
\hline & & $z$ & $\mathrm{TF}_{z}$ & 21 & 34.85 & 0.027 & & $34.88 \pm 0.06$ & $94.48 \pm 2.61$ & $77.87 \pm 2.33$ \\
\hline & & $z$ & $\mathrm{TF}_{z c}$ & 20 & 34.84 & 0.007 & & $34.85 \pm 0.06$ & $93.19 \pm 2.57$ & $78.95 \pm 2.36$ \\
\hline & & $z$ & $\mathrm{TF}_{z \mu}$ & 21 & 34.84 & -0.002 & & $34.84 \pm 0.06$ & $92.80 \pm 2.56$ & $79.28 \pm 2.37$ \\
\hline & & $W 1$ & $\mathrm{TF}_{W 1}$ & 23 & 34.90 & 0.011 & & $34.91 \pm 0.08$ & $96.00 \pm 3.54$ & $76.63 \pm 2.96$ \\
\hline & & $W 1$ & $\mathrm{TF}_{W 1 c}$ & 20 & 34.80 & 0.016 & 0.04 & $34.86 \pm 0.06$ & $93.60 \pm 2.59$ & $78.60 \pm 2.35$ \\
\hline & & $W 1$ & $\mathrm{TF}_{W 1 \mu}$ & 23 & 34.75 & -0.009 & 0.11 & $34.85 \pm 0.07$ & $93.35 \pm 3.01$ & $78.81 \pm 2.70$ \\
\hline & & $W 2$ & $\mathrm{TF}_{W 2}$ & 23 & 34.90 & 0.011 & & $34.91 \pm 0.09$ & $95.97 \pm 3.98$ & $76.66 \pm 3.30$ \\
\hline & & $W 2$ & $\mathrm{TF}_{W 2 c}$ & 20 & 34.79 & 0.003 & 0.06 & $34.85 \pm 0.06$ & $93.46 \pm 2.58$ & $78.72 \pm 2.36$ \\
\hline & & $W 2$ & $\mathrm{TF}_{W 2 \mu}$ & 23 & 34.70 & -0.017 & 0.17 & $34.85 \pm 0.08$ & $93.47 \pm 3.44$ & $78.71 \pm 3.04$ \\
\hline \multirow[t]{21}{*}{ NGC 4065} & \multirow[t]{21}{*}{$7501 \pm 63$} & $u$ & $\mathrm{TF}_{u}$ & 14 & 35.11 & 0.055 & & $35.16 \pm 0.10$ & $107.89 \pm 4.97$ & $69.52 \pm 3.25$ \\
\hline & & $u$ & $\mathrm{TF}_{\mathrm{uc} 1}$ & 14 & 35.15 & 0.033 & & $35.18 \pm 0.08$ & $108.80 \pm 4.01$ & $68.95 \pm 2.61$ \\
\hline & & $u$ & $\mathrm{TF}_{\mathrm{uc} 2}$ & 12 & 35.11 & 0.035 & & $35.15 \pm 0.10$ & $106.91 \pm 4.92$ & $70.16 \pm 3.28$ \\
\hline & & $g$ & $\mathrm{TF}_{g}$ & 14 & 35.12 & 0.028 & & $35.15 \pm 0.07$ & $107.04 \pm 3.45$ & $70.08 \pm 2.33$ \\
\hline & & $g$ & $\mathrm{TF}_{\mathrm{gc} 1}$ & 14 & 35.16 & 0.044 & & $35.20 \pm 0.07$ & $109.83 \pm 3.54$ & $68.30 \pm 2.28$ \\
\hline & & $g$ & $\mathrm{TF}_{\mathrm{gc} 2}$ & 12 & 35.12 & 0.040 & & $35.16 \pm 0.09$ & $107.65 \pm 4.46$ & $69.68 \pm 2.95$ \\
\hline & & $r$ & $\mathrm{TF}_{r}$ & 14 & 35.12 & 0.026 & & $35.15 \pm 0.07$ & $106.97 \pm 3.45$ & $70.12 \pm 2.34$ \\
\hline & & $r$ & $\mathrm{TF}_{r c}$ & 12 & 35.10 & 0.008 & & $35.11 \pm 0.08$ & $105.09 \pm 3.87$ & $71.37 \pm 2.70$ \\
\hline & & $r$ & $\mathrm{TF}_{r \mu}$ & 14 & 35.10 & 0.013 & & $35.11 \pm 0.07$ & $105.32 \pm 3.40$ & $71.22 \pm 2.37$ \\
\hline & & $i$ & $\mathrm{TF}_{i}$ & 14 & 35.11 & 0.025 & & $35.14 \pm 0.08$ & $106.43 \pm 3.92$ & $70.48 \pm 2.66$ \\
\hline & & $i$ & $\mathrm{TF}_{i c}$ & 12 & 35.08 & 0.029 & & $35.11 \pm 0.08$ & $105.16 \pm 3.87$ & $71.33 \pm 2.70$ \\
\hline & & $i$ & $\mathrm{TF}_{i \mu}$ & 14 & 35.08 & 0.026 & & $35.11 \pm 0.08$ & $104.99 \pm 3.87$ & $71.45 \pm 2.70$ \\
\hline & & $z$ & $\mathrm{TF}_{z}$ & 14 & 35.13 & 0.024 & & $35.15 \pm 0.08$ & $107.35 \pm 3.95$ & $69.87 \pm 2.64$ \\
\hline & & $z$ & $\mathrm{TF}_{z c}$ & 12 & 35.09 & 0.006 & & $35.10 \pm 0.08$ & $104.52 \pm 3.85$ & $71.77 \pm 2.71$ \\
\hline & & $z$ & $\mathrm{TF}_{z \mu}$ & 14 & 35.11 & -0.002 & & $35.11 \pm 0.08$ & $105.11 \pm 3.87$ & $71.37 \pm 2.70$ \\
\hline & & $W 1$ & $\mathrm{TF}_{W 1}$ & 12 & 35.06 & 0.012 & & $35.07 \pm 0.11$ & $103.38 \pm 5.24$ & $72.56 \pm 3.73$ \\
\hline & & $W 1$ & $\mathrm{TF}_{W 1 c}$ & 12 & 35.04 & 0.022 & 0.04 & $35.10 \pm 0.08$ & $104.82 \pm 3.86$ & $71.56 \pm 2.70$ \\
\hline & & $W 1$ & $\mathrm{TF}_{W 1 \mu}$ & 12 & 34.98 & -0.013 & 0.11 & $35.08 \pm 0.10$ & $103.61 \pm 4.77$ & $72.40 \pm 3.39$ \\
\hline & & $W 2$ & $\mathrm{TF}_{W 2}$ & 12 & 35.08 & 0.012 & & $35.09 \pm 0.12$ & $104.33 \pm 5.77$ & $71.90 \pm 4.02$ \\
\hline & & $W 2$ & $\mathrm{TF}_{W 2 c}$ & 12 & 35.04 & 0.004 & 0.06 & $35.10 \pm 0.08$ & $104.91 \pm 3.86$ & $71.50 \pm 2.70$ \\
\hline & & $W 2$ & $\mathrm{TF}_{W 2 \mu}$ & 12 & 34.95 & -0.020 & 0.17 & $35.10 \pm 0.10$ & $104.69 \pm 4.82$ & $71.65 \pm 3.35$ \\
\hline \multirow[t]{4}{*}{ A539 } & \multirow[t]{4}{*}{$8995 \pm 87$} & $W 1$ & $\mathrm{TF}_{W 1}$ & 22 & 35.22 & -0.004 & & $35.22 \pm 0.08$ & $110.48 \pm 4.07$ & $81.41 \pm 3.10$ \\
\hline & & $W 1$ & $\mathrm{TF}_{W 1 \mu}$ & 22 & 35.17 & -0.037 & 0.11 & $35.24 \pm 0.06$ & $111.86 \pm 3.09$ & $80.41 \pm 2.35$ \\
\hline & & $W 2$ & $\mathrm{TF}_{W 2}$ & 22 & 35.14 & 0.002 & & $35.14 \pm 0.09$ & $106.77 \pm 4.43$ & $84.24 \pm 3.59$ \\
\hline & & $W 2$ & $\mathrm{TF}_{W 2 \mu}$ & 22 & 35.07 & -0.053 & 0.17 & $35.19 \pm 0.07$ & $108.97 \pm 3.51$ & $82.54 \pm 2.78$ \\
\hline \multirow[t]{10}{*}{ A2634/66 } & \multirow[t]{10}{*}{$8954 \pm 98$} & $u$ & $\mathrm{TF}_{u}$ & 29 & 35.45 & 0.061 & & $35.51 \pm 0.08$ & $126.53 \pm 4.66$ & $70.77 \pm 2.72$ \\
\hline & & $u$ & $\mathrm{TF}_{\mathrm{uc} 1}$ & 29 & 35.33 & 0.032 & & $35.36 \pm 0.06$ & $118.13 \pm 3.26$ & $75.80 \pm 2.25$ \\
\hline & & $u$ & $\mathrm{TF}_{\mathrm{uc} 2}$ & 26 & 35.36 & 0.036 & & $35.40 \pm 0.07$ & $120.01 \pm 3.87$ & $74.61 \pm 2.54$ \\
\hline & & $g$ & $\mathrm{TF}_{g}$ & 29 & 35.34 & 0.033 & & $35.37 \pm 0.07$ & $118.75 \pm 3.83$ & $75.40 \pm 2.57$ \\
\hline & & $g$ & $\mathrm{TF}_{\mathrm{gcl} 1}^{8}$ & 29 & 35.33 & 0.052 & & $35.38 \pm 0.06$ & $119.24 \pm 3.29$ & $75.09 \pm 2.23$ \\
\hline & & $g$ & $\mathrm{TF}_{\mathrm{gc} 2}$ & 26 & 35.34 & 0.051 & & $35.39 \pm 0.06$ & $119.72 \pm 3.31$ & $74.79 \pm 2.22$ \\
\hline & & $r$ & $\mathrm{TF}_{r}$ & 29 & 35.31 & 0.031 & & $35.34 \pm 0.06$ & $117.02 \pm 3.23$ & $76.52 \pm 2.27$ \\
\hline & & $r$ & $\mathrm{TF}_{r c}$ & 26 & 35.31 & 0.011 & & $35.32 \pm 0.06$ & $115.95 \pm 3.20$ & $77.22 \pm 2.30$ \\
\hline & & $r$ & $\mathrm{TF}_{r \mu}$ & 29 & 35.29 & 0.018 & & $35.31 \pm 0.06$ & $115.23 \pm 3.18$ & $77.71 \pm 2.31$ \\
\hline & & $i$ & $\mathrm{TF}_{i}$ & 29 & 35.26 & 0.030 & & $35.29 \pm 0.06$ & $114.27 \pm 3.16$ & $78.36 \pm 2.33$ \\
\hline
\end{tabular}


Table 10

(Continued)

\begin{tabular}{|c|c|c|c|c|c|c|c|c|c|c|}
\hline \multirow[t]{12}{*}{ (1) } & $\begin{array}{c}V_{\text {mod }} \\
\mathrm{km} \mathrm{s}^{-1} \\
(2)\end{array}$ & $\begin{array}{l}\text { TFR } \\
\text { Band } \\
(3)\end{array}$ & $\begin{array}{l}\text { TFR } \\
\text { Model } \\
(4)\end{array}$ & Ngal & $\begin{array}{c}\mathrm{DM}_{o} \\
\mathrm{mag} \\
(6)\end{array}$ & $\begin{array}{l}\text { Bias } \\
\text { mag } \\
(7)\end{array}$ & $\begin{array}{c}\Delta \mathrm{DM} \\
\mathrm{mag} \\
(8)\end{array}$ & $\begin{array}{c}\mathrm{DM}_{c} \\
\mathrm{mag} \\
(9)\end{array}$ & $\begin{array}{c}D \\
\mathrm{Mpc} \\
(10)\end{array}$ & $\begin{array}{c}V_{\mathrm{mod}} / D \\
\mathrm{~km} \mathrm{~s}^{-1} \mathrm{Mpc}^{-1} \\
(11)\end{array}$ \\
\hline & & $i$ & $\mathrm{TF}_{i c}$ & 26 & 35.26 & 0.036 & & $35.30 \pm 0.06$ & $114.62 \pm 3.17$ & $78.12 \pm 2.32$ \\
\hline & & $i$ & $\mathrm{TF}_{i \mu}$ & 29 & 35.24 & 0.031 & & $35.27 \pm 0.06$ & $113.29 \pm 3.13$ & $79.04 \pm 2.35$ \\
\hline & & $z$ & $\mathrm{TF}_{z}$ & 29 & 35.24 & 0.027 & & $35.27 \pm 0.06$ & $113.08 \pm 3.12$ & $79.19 \pm 2.35$ \\
\hline & & $z$ & $\mathrm{TF}_{z c}$ & 26 & 35.23 & 0.007 & & $35.24 \pm 0.06$ & $111.53 \pm 3.08$ & $80.29 \pm 2.39$ \\
\hline & & $z$ & $\mathrm{TF}_{z \mu}$ & 29 & 35.23 & -0.002 & & $35.23 \pm 0.06$ & $111.06 \pm 3.07$ & $80.63 \pm 2.40$ \\
\hline & & $W 1$ & $\mathrm{TF}_{W 1}$ & 26 & 35.28 & 0.011 & & $35.29 \pm 0.08$ & $114.34 \pm 4.21$ & $78.31 \pm 3.01$ \\
\hline & & $W 1$ & $\mathrm{TF}_{W 1 c}$ & 26 & 35.21 & 0.027 & 0.04 & $35.28 \pm 0.06$ & $113.62 \pm 3.14$ & $78.81 \pm 2.34$ \\
\hline & & $W 1$ & $\mathrm{TF}_{W 1 \mu}$ & 26 & 35.13 & -0.017 & 0.11 & $35.22 \pm 0.07$ & $110.82 \pm 3.57$ & $80.80 \pm 2.75$ \\
\hline & & $W 2$ & $\mathrm{TF}_{W 2}$ & 26 & 35.27 & 0.013 & & $35.28 \pm 0.09$ & $113.92 \pm 4.72$ & $78.60 \pm 3.37$ \\
\hline & & $W 2$ & $\mathrm{TF}_{W 2 c}$ & 26 & 35.21 & 0.006 & 0.06 & $35.28 \pm 0.06$ & $113.53 \pm 3.14$ & $78.87 \pm 2.34$ \\
\hline & & $W 2$ & $\mathrm{TF}_{W 2 \mu}$ & 26 & 35.07 & -0.027 & 0.17 & $35.21 \pm 0.08$ & $110.33 \pm 4.06$ & $81.16 \pm 3.12$ \\
\hline \multirow[t]{21}{*}{ A2151 (Hercules) } & $11353 \pm 121$ & $u$ & $\mathrm{TF}_{u}$ & 39 & 35.86 & 0.083 & & $35.94 \pm 0.07$ & $154.40 \pm 4.98$ & $73.53 \pm 2.50$ \\
\hline & & $u$ & $\mathrm{TF}_{\mathrm{uc} 1}$ & 39 & 35.92 & 0.054 & & $35.97 \pm 0.06$ & $156.60 \pm 4.33$ & $72.49 \pm 2.15$ \\
\hline & & $u$ & $\mathrm{TF}_{\mathrm{uc} 2}$ & 33 & 35.97 & 0.061 & & $36.03 \pm 0.07$ & $160.74 \pm 5.18$ & $70.63 \pm 2.40$ \\
\hline & & $g$ & $\mathrm{TF}_{g}$ & 39 & 35.87 & 0.031 & & $35.90 \pm 0.06$ & $151.42 \pm 4.18$ & $74.98 \pm 2.22$ \\
\hline & & $g$ & $\mathrm{TF}_{\mathrm{gc} 1}$ & 39 & 35.95 & 0.050 & & $36.00 \pm 0.06$ & $158.50 \pm 4.38$ & $71.63 \pm 2.12$ \\
\hline & & $g$ & $\mathrm{TF}_{\mathrm{gc} 2}$ & 33 & 35.95 & 0.048 & & $36.00 \pm 0.07$ & $158.37 \pm 5.11$ & $71.68 \pm 2.43$ \\
\hline & & $r$ & $\mathrm{TF}_{r}$ & 39 & 35.91 & 0.029 & & $35.94 \pm 0.06$ & $154.11 \pm 4.26$ & $73.67 \pm 2.18$ \\
\hline & & $r$ & $\mathrm{TF}_{r c}$ & 33 & 35.90 & 0.010 & & $35.91 \pm 0.07$ & $152.03 \pm 4.90$ & $74.68 \pm 2.54$ \\
\hline & & $r$ & $\mathrm{TF}_{r \mu}$ & 39 & 35.88 & 0.015 & & $35.89 \pm 0.06$ & $151.00 \pm 4.17$ & $75.18 \pm 2.23$ \\
\hline & & $i$ & $\mathrm{TF}_{i}$ & 39 & 35.91 & 0.028 & & $35.94 \pm 0.06$ & $154.04 \pm 4.26$ & $73.70 \pm 2.18$ \\
\hline & & $i$ & $\mathrm{TF}_{i c}$ & 33 & 35.89 & 0.033 & & $35.92 \pm 0.07$ & $153.00 \pm 4.93$ & $74.20 \pm 2.52$ \\
\hline & & $i$ & $\mathrm{TF}_{i \mu}$ & 39 & 35.87 & 0.029 & & $35.90 \pm 0.06$ & $151.28 \pm 4.18$ & $75.05 \pm 2.22$ \\
\hline & & $z$ & $\mathrm{TF}_{z}$ & 39 & 35.95 & 0.025 & & $35.97 \pm 0.06$ & $156.67 \pm 4.33$ & $72.47 \pm 2.15$ \\
\hline & & $z$ & $\mathrm{TF}_{z c}$ & 33 & 35.92 & 0.006 & & $35.93 \pm 0.07$ & $153.20 \pm 4.94$ & $74.11 \pm 2.52$ \\
\hline & & $z$ & $\mathrm{TF}_{z \mu}$ & 39 & 35.90 & -0.002 & & $35.90 \pm 0.06$ & $151.23 \pm 4.18$ & $75.07 \pm 2.22$ \\
\hline & & $W 1$ & $\mathrm{TF}_{W 1}$ & 33 & 36.04 & 0.011 & & $36.05 \pm 0.09$ & $162.28 \pm 6.73$ & $69.96 \pm 2.99$ \\
\hline & & $W 1$ & $\mathrm{TF}_{W 1 c}$ & 33 & 35.85 & 0.025 & 0.04 & $35.91 \pm 0.07$ & $152.40 \pm 4.91$ & $74.49 \pm 2.53$ \\
\hline & & $W 1$ & $\mathrm{TF}_{W 1 \mu}$ & 33 & 35.88 & -0.016 & 0.11 & $35.97 \pm 0.08$ & $156.59 \pm 5.77$ & $72.50 \pm 2.78$ \\
\hline & & $W 2$ & $\mathrm{TF}_{W 2}$ & 33 & 36.04 & 0.013 & & $36.05 \pm 0.09$ & $162.40 \pm 6.73$ & $69.91 \pm 2.99$ \\
\hline & & $W 2$ & $\mathrm{TF}_{W 2 c}$ & 33 & 35.85 & 0.005 & 0.06 & $35.92 \pm 0.07$ & $152.42 \pm 4.91$ & $74.49 \pm 2.53$ \\
\hline & & $W 2$ & $\mathrm{TF}_{W 2 \mu}$ & 33 & 35.82 & -0.027 & 0.17 & $35.96 \pm 0.08$ & $155.83 \pm 5.74$ & $72.85 \pm 2.79$ \\
\hline
\end{tabular}

(This table is available in machine-readable form.)

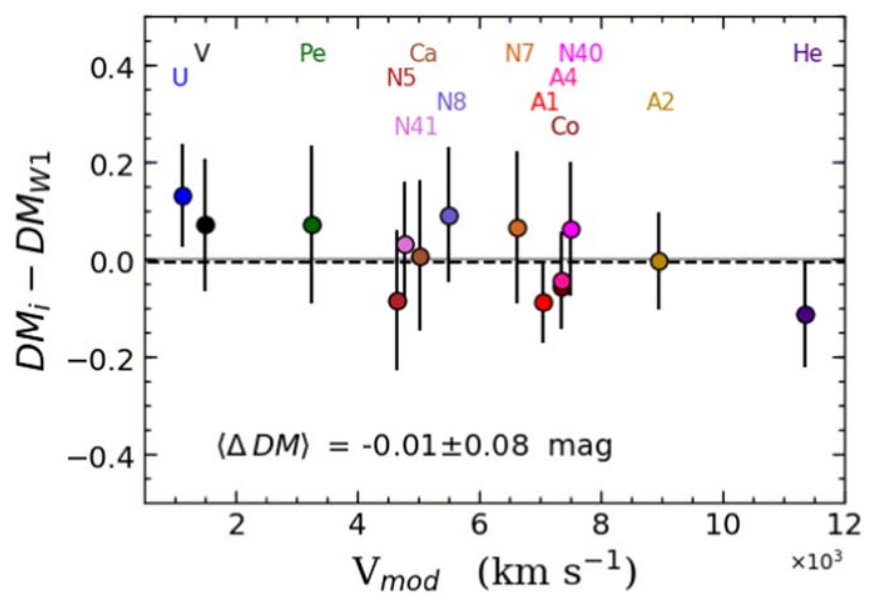

Figure 24. Differences between the measured cluster distance moduli at the $i$ and $W 1$ bands vs. their radial velocities, $V_{\text {mod. }}$ The cluster codes are horizontally placed based on the corresponding $V_{\text {mod }}$ values. The horizontal axis is the cosmologically corrected radial velocity of clusters in the CMB rest frame. The dashed horizontal line displays the average of the DM offset, $\langle\Delta \mathrm{DM}\rangle$, calculated by incorporating the vertical error bars. with the most significant correlations to ITFR residuals, hence most capable of reducing the scatter in adjusted ITFR relations, to be $\Delta\left(i^{*}-W 1^{*}\right)$ and $\Delta\left(\left\langle\mu_{\lambda^{*}}\right\rangle_{e}\right)$. The correlations with these observables in the case of the $i$ band are shown in Figure 20. The adjusted ITFRs based on these models are labeled with the codes $\mathrm{TF}_{\lambda c}$ and $\mathrm{TF}_{\lambda \mu}$, respectively, where $\lambda$ is replaced with $r, i$, and $z$.

All of the correlations at the $r, i$, and $z$ bands are weak. Comparing the lower panels of Figures 18 and 20, the slopes of the correlations with colors $\lambda^{\star}-W 1^{\star}$ transition from positive to negative, flattening to zero slope between the $z$ and $W 1$ bands. Adjustments coupled to color parameters do little to improve the TFR scatter (see Table 9 and Figure 21 in the case of the $i$ band). Likewise, correlation coefficients related to surface brightness or H I pseudocolors are small. We favor not making third-parameter adjustments to the TFR at the $r, i$, and $z$ bands, since adding parameters adds uncertainties.

\subsection{Modified ITFRs at Infrared $\mathrm{W} 1$ and $\mathrm{W} 2$ Bands}

The effectiveness of the optical-infrared colors to reduce the scatters of the unadjusted ITFRs at infrared bands have already 
been noticed in previous studies. For example, Sorce et al. (2013) observed a significant correlation between the I- IRAC [3.6] color and deviations from the mean ITFR constructed at the infrared IRAC [3.6] band. Inspired by their work, Neill et al. (2014) applied the same methodology to reduce the scatter about the WISE $W 1$ and $W 2$ ITFRs.

Giving consideration to a similar color correlation with the present data, Table 8 lists the linear dependencies of unadjusted $W 1$ and $W 2$ ITFR residuals on the $i^{*}-W 1^{*}$ and $i^{*}-W 2^{*}$ parameters. We find similar trends as previous studies; however, the correlations are not very significant and cannot usefully reduce the scatter of the ITFR. ${ }^{15}$ Instead, we consider $\Delta\left(i^{*}-W 1^{*}\right)$ and $\Delta\left(i^{*}-W 2^{*}\right)$ color terms that are in higher correlation with the ITFR residuals, with correlation factors of $53 \%$ and $59 \%$, respectively. In addition, the surface brightness seems to be capable of reducing the scatter efficiently.

Figure 22 displays the deviations from the $W 1$-band ITFR versus $\Delta\left(i^{*}-W 1^{*}\right)$ and $\Delta\left(\left\langle\mu_{\left.W 1^{*}\right\rangle_{e}}\right)\right.$. In both cases, the slopes and zero-points of the fitted linear relations are significant. Analogous relations have been derived for the $W 2$ band. The middle and bottom panels of Figure 23 plot the revised luminosity-line width relations after applying corrections based on the $\Delta\left(i^{*}-W 1^{*}\right)$ and $\Delta\left(\left\langle\mu_{\left.W 1^{*}\right\rangle_{e}}\right)\right.$ parameters, labeled $\mathrm{TF}_{W 1 c}$ and $\mathrm{TF}_{W 1 \mu}$, respectively. In addition, the adjustments using additional parameters reduce the curvature of the best fit after the break point at $\log \left(W_{m x}^{i}\right)=2.4$. This reduction is in agreement with the results of Neill et al. (2014), where no curvature for the relation of the color-adjusted magnitude with line width is apparent. The larger correlation factor of $\Delta\left(i^{*}-W 1^{*}\right)(53 \%)$ compared to that of $\Delta\left(\left\langle\mu_{\left.W 1^{*}\right\rangle_{e}}\right)(38 \%)\right.$ translates to greater reduction in the scatter about the adjusted TF model. The $0.58 \mathrm{mag}$ rms scatter of $\mathrm{TF}_{W 1}$ is lowered to 0.47 and 0.51 mag for the $\mathrm{TF}_{W 1 c}$ and $\mathrm{TF}_{W 1 \mu}$ cases, respectively.

Ever since Aaronson et al. (1979), it has been anticipated that minimal scatter in the TFR would be achieved at infrared bands. Indeed, Ponomareva et al. (2017) explored correlations in 12 bands from the far-ultraviolet to $4.5 \mu \mathrm{m}$ and found the tightest fit at $3.6 \mu \mathrm{m}$. With extensive modeling, adjustments can be made to account for radiation from dust associated with starforming regions that can contribute $10 \%-30 \%$ of light at 3.4-3.6 $\mu \mathrm{m}$ (Querejeta et al. 2015). In any event, the minimization in scatter found by Ponomareva et al. (2017) and similarly by Lelli et al. (2016) refers to the scatter orthogonal to mean bivariate fits. The scatter relevant for the acquisition of distances is that in magnitudes and is aggravated by the steepening of slopes toward the infrared.

\subsection{The Baryonic TFR}

McGaugh (2005) pointed out that the fractional representation of interstellar gas mass increases progressively toward galaxies of lower stellar mass. He suggested that luminosity be replaced by a linear combination of luminosity and the $\mathrm{H}$ I flux as a measure of the galactic baryon content, $M_{b}$,

$$
M_{b}=1.33 M_{\mathrm{H} \mathrm{I}}+\Upsilon_{\star} L_{\lambda},
$$

where $\Upsilon_{\star}$ is the stellar mass-to-light ratio $\left(\sim 0.5 M_{\odot} / L_{\odot}\right.$ at $\lambda=3.6 \mu \mathrm{m}$ ) and the multiplier 1.33 on $M_{\mathrm{H} \mathrm{I}}$ accounts for the unseen contribution of helium (Lelli et al. 2019).

\footnotetext{
${ }^{15}$ Similarly, Ponomareva et al. (2017) found that the color term in a bivariate solution for the luminosity-line width relation at the IRAC [3.6] band did not rise to a level of significance.
}
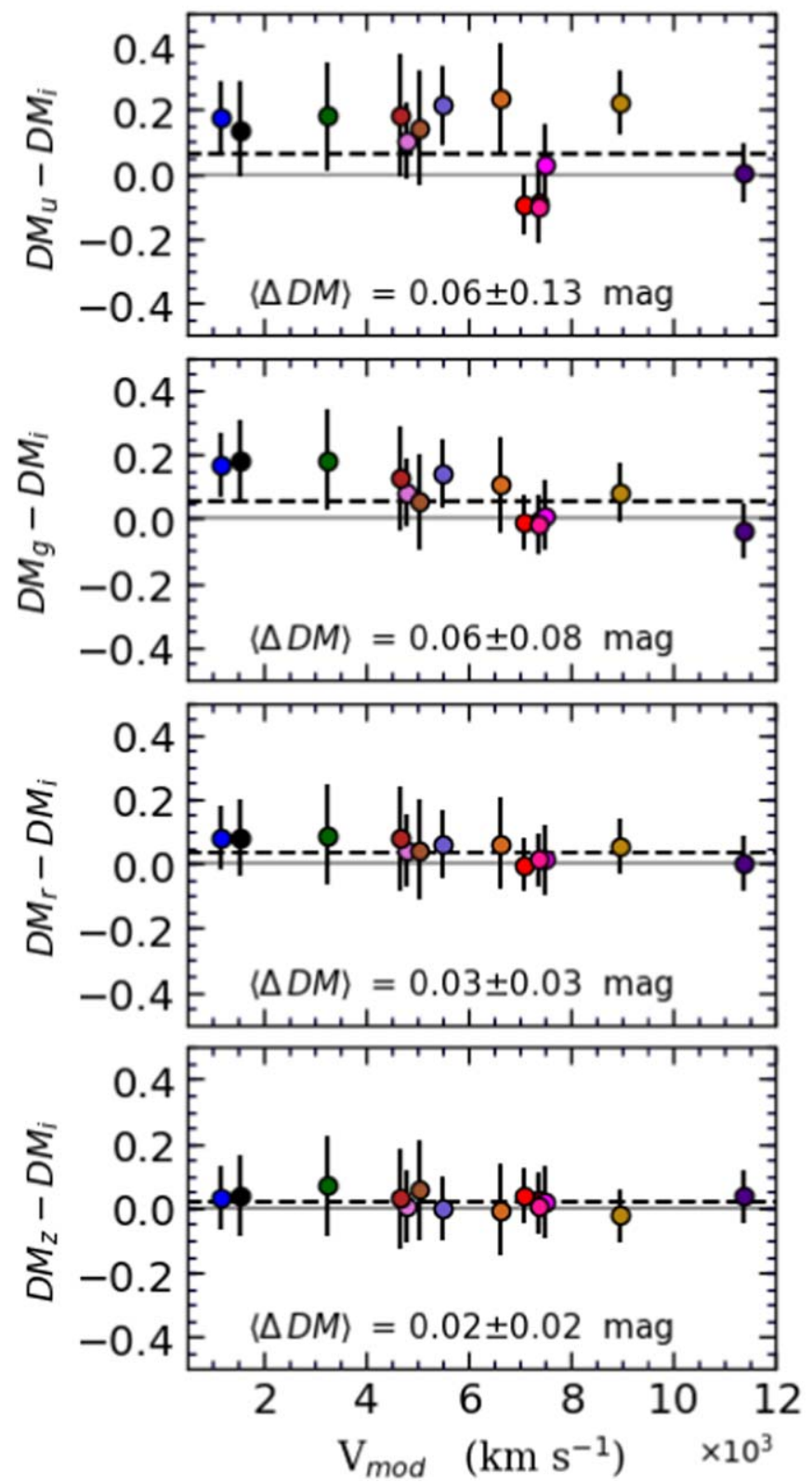

Figure 25. Offset of cluster distance moduli, DM, measured at the optical $u, g$, $r$, and $z$ bands from those measured at the $i$ band using the unadjusted TFR relations. The color scheme and other details are the same as in Figure 24.

The baryonic formulation tends to flatten the TFR by raising the low line width end relative to the high line width end and may somewhat mitigate curvature (Noordermeer \& Verheijen 2007). Nevertheless, we have not given the baryonic TFR close attention. The neutral gas only becomes a substantial component of the baryonic mass of a galaxy in the vicinity of our faint cutoff at $M_{i}=-17$. In particular, though, we are not confident of the reliability of the $\mathrm{H}$ I flux parameters in our H I catalogs at the EDD. Ponomareva et al. (2016) noted the basis of our concerns. In the compilation of the ADHI, great care was given to the accumulation of line widths on a coherent system, but $\mathrm{H}$ I fluxes have not received such attention. Contributions come from a variety of telescopes with beams of different sizes that may or may not capture most of the H I flux. Inhomogeneities in flux measurements might depend on the 

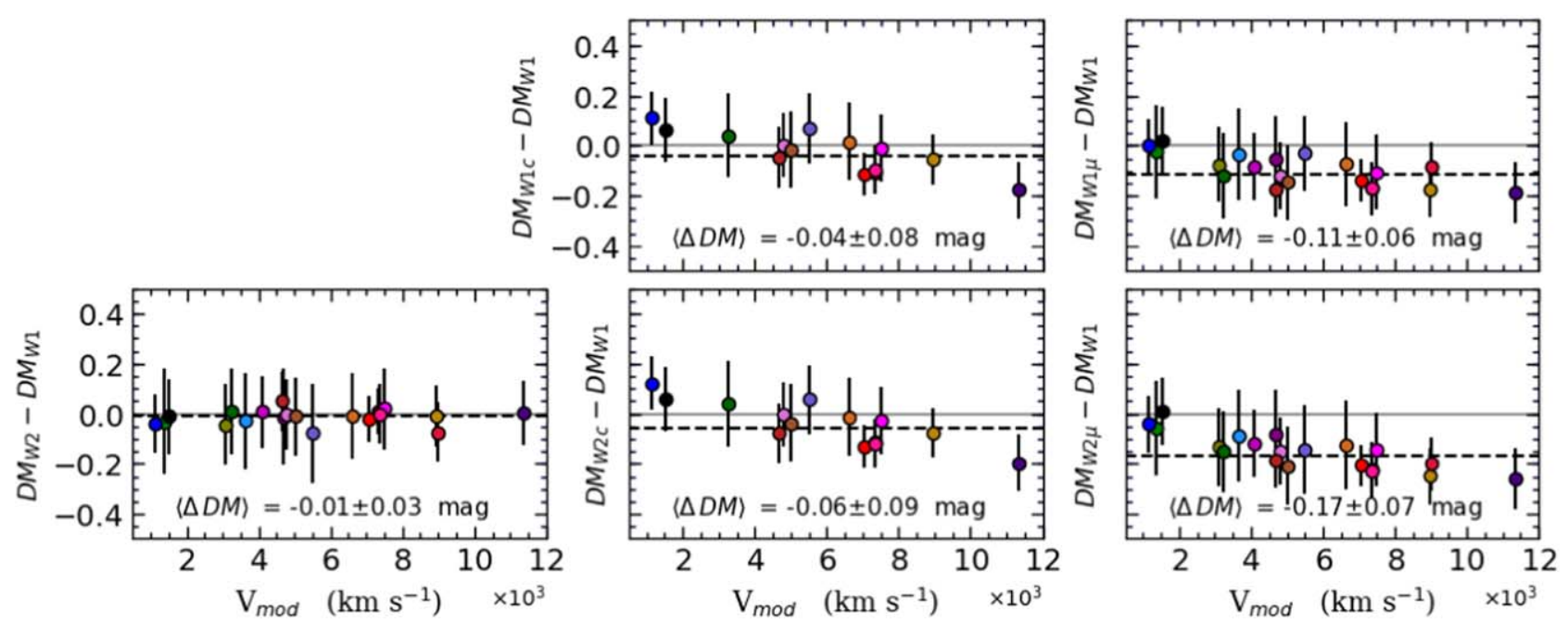

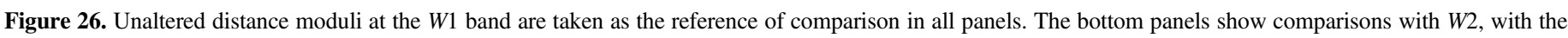

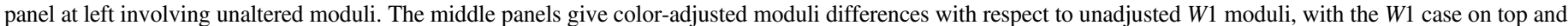

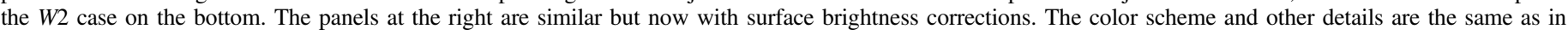
Figure 24.

telescope, and hence portions of the sky, and on target sizes, and hence distance.

\section{Cluster Radial Velocities}

The mean radial velocity of each cluster is determined by averaging the velocities of all known constituents (not just those with distance measurements) using the biweight statistics discussed in Beers et al. (1990). For clusters beyond $3500 \mathrm{~km} \mathrm{~s}^{-1}$, memberships are based on the 2MASS galaxy group catalog of Tully (2015), while within $3500 \mathrm{~km} \mathrm{~s}^{-1}$, we draw on the group catalog of Kourkchi \& Tully (2017). The average radial velocities of clusters are then shifted to the cosmic microwave background (CMB) rest frame $V_{\mathrm{cmb}}$ and further adjusted for cosmological curvature $V_{\text {mod }}=f V_{\mathrm{cmb}}$, where

$$
f=1+\frac{1}{2}\left[1-q_{0}\right] z-\frac{1}{6}\left[1-q_{0}-3 q_{0}^{2}+j_{0}\right] z^{2},
$$

the jerk parameter $j_{0}=1$, and the acceleration parameter $q_{0}=$ $\frac{1}{2}\left(\Omega_{m}-2 \Omega_{\Lambda}\right)=-0.595$, assuming $\Omega_{m}=0.27, \Omega_{m}+\Omega_{\Lambda}=1$. These cosmological adjustments are negligible for the nearby clusters, reaching in the extreme 3\% for the Hercules cluster. The second column of Table 10 lists these adjusted radial velocities, $V_{\text {mod }}$, and their uncertainties.

\section{Distances of Clusters}

A product of the TFR calibration is the distance moduli of the galaxy clusters used to form the TFR template. The raw distance moduli of clusters, $\mathrm{DM}_{o}$, are listed in column (6) of Table 10 for different TFR models listed in column (4). Column (7) contains $b$, the luminosity function scatter bias combined with the effect of TFR curvature that is calculated following the discussion presented in Section 3.6. Column (8) contains any other adjustment bias that will be discussed later in this section, $\triangle \mathrm{DM}$. Column (9) tabulates the corrected distance moduli, given as $\mathrm{DM}_{c}=\mathrm{DM}_{o}+b+\Delta \mathrm{DM}$, that are converted to distances in units of $\mathrm{Mpc}$ and presented in column (10).
Initially, we set $\Delta D M=0$, and we plot the differences of the bias-corrected moduli as a function of the cluster radial velocities to test the consistency of the measurements at different passbands. At optical bands, 14 galaxy clusters contributed in our calibration process, while in the infrared, 20 clusters are available. First, we check the consistency of our distance measurements between the optical and infrared bands using the $i$ and $W 1$ bands as proxies, and then we evaluate the agreements within the optical and infrared bands separately.

Figure 24 plots the differences of the distance moduli measured at the $i$ and $W 1$ bands for the 14 clusters in common. No significant offset is apparent. We notice positive offsets for the nearest three clusters, Virgo, Ursa Major, and Fornax, and a negative offset for the most distant Hercules cluster. After further exploration, we find that the Virgo galaxies are slightly redder and the Hercules galaxies are bluer compared to the average color distribution of the template galaxies used in the calibration process. Redder galaxies appear to be more luminous at longer wavelengths, and therefore they are located at smaller distances. The opposite effect is true for galaxies that are in general bluer than average. This subtle effect was seen earlier (Tully \& Pierce 2000; Neill et al. 2014) with the very nearby clusters, in the first case with entirely distinct optical and infrared photometry and in the second case with distinct optical photometry.

We look for offsets of the cluster moduli, measured using the unadjusted TFRs ( $\mathrm{TF}_{\lambda}$ models), at optical passbands relative to that at the $i$ band in Figure 25. It is seen that systematic differences in moduli are highest for the $u$ band and decrease toward longer wavelengths. These offsets are not statistically significant; nonetheless, we offer adjustments at the $u$ and $g$ bands so moduli in these bands are on the same scale as the $i$ band. In our final analysis, though, we do not make use of the $u$ and $g$ moduli. By contrast, we see in Figure 25 that the moduli at $r, i$, and $z$ are in close agreement without any modifications. Going forward, we only use the $r, i$, and $z$ relations without relative bandpass adjustments to measure distances with optical photometry.

For evaluation of the infrared distances, we choose the $W 1$ band as a reference. In order to measure distances at this 

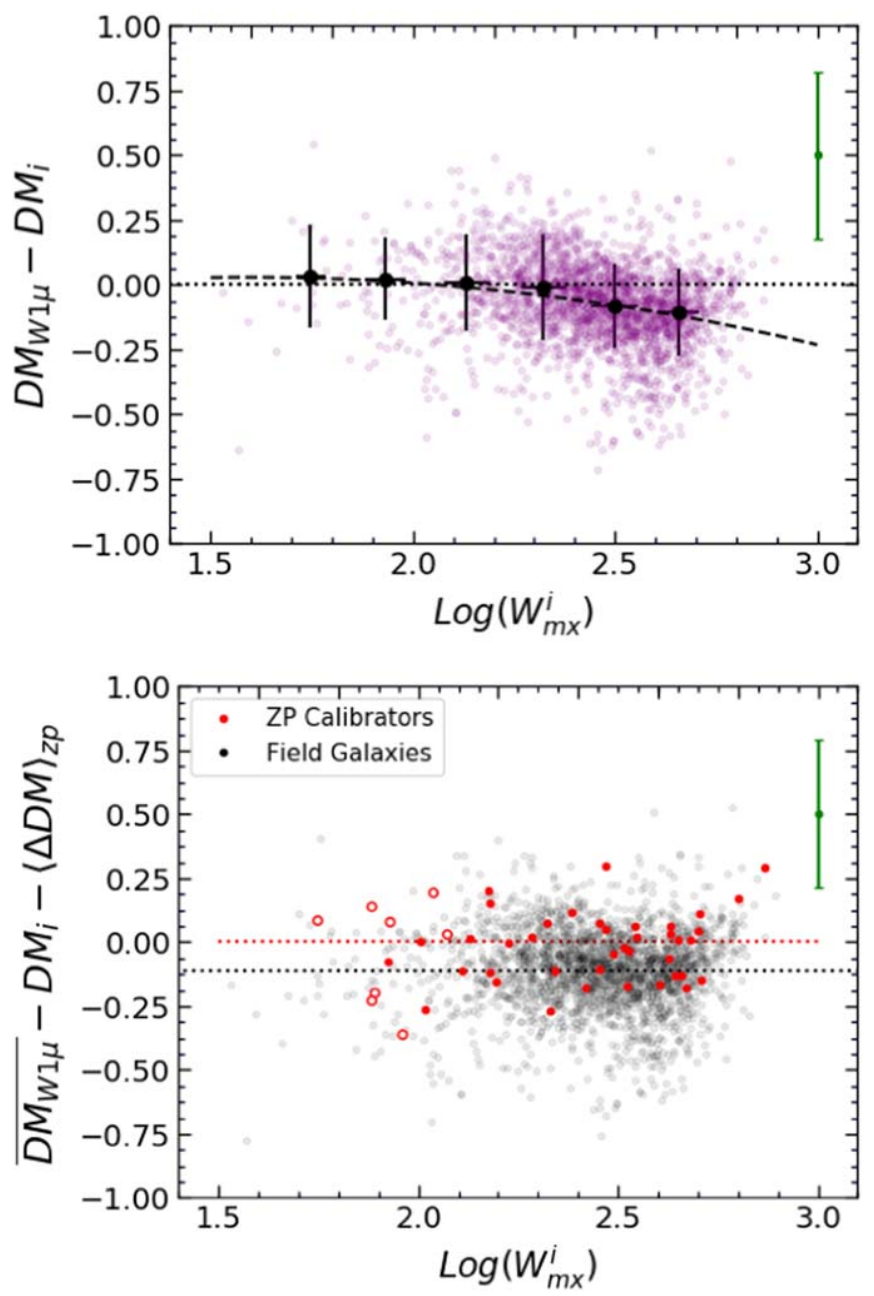

Figure 27. Top: differences in distance moduli of field galaxies, DM, between the $\mathrm{TF}_{W 1 \mu}$ and $\mathrm{TF}_{i}$ cases. Each purple point represents a galaxy, with black points showing averages in horizontal bins of 0.2 . The black dashed line is the best quadratic fitted function on all data points, minimizing vertical residuals, with expression $\mathrm{DM}_{W 1 \mu}-\mathrm{DM}_{i}=(-0.13 \pm 0.06) X^{2}+(0.42 \pm 0.28) X+$ $(-0.31 \pm 0.33)$, where $X=\log W_{m x}^{i}$. Bottom: offset of distance moduli after correcting $\mathrm{DM}_{W 1 \mu}$ for the line width-dependent bias presented in the top panel, $\overline{\mathrm{DM}_{W 1 \mu}}-\mathrm{DM}_{i}$. The zero-point of the plot is set at $\langle\Delta \mathrm{DM}\rangle_{z \mathrm{p}}=0.11 \mathrm{mag}$, the average of the moduli differences of the zero-point calibrators, displayed as red filled points. Gray points represent field galaxies, while red open points are rejected faint calibrators. The black dotted line at -0.11 is at the average moduli offset of field galaxies. Green error bars at the top right give typical differences for field galaxies.

infrared band, we apply the adjusted ITFRs that have smaller scatters. As discussed in Section 4.5, both $\Delta\left(i^{*}-W 1^{*}\right)$ and $\Delta\left(\left\langle\mu_{\left.W 1^{*}\right\rangle_{e}}\right)\right.$ parameters are capable of reducing the scatter. However, it is more practical for our purposes to use the corrections based on the $W 1$ surface brightness because we sometimes lack optical photometry.

Figure 26 displays the differences of the cluster moduli at adjusted and unadjusted infrared bands with reference to the $W 1$ band. The left panel of this plot shows an excellent consistency between the $W 1$ and $W 2$ results. The middle panels of Figure 26 show that the color-adjusted models, $\mathrm{TF}_{W 1 c}$ and $\mathrm{TF}_{W 2 c}$, result in slightly smaller distance moduli compared to that measured using the unadjusted models. The right panels of Figure 26 reveal more significant offsets for the $\mathrm{TF}_{W 1 \mu}$ and $\mathrm{TF}_{W 2 \mu}$ moduli, calling for adjustments to the distance moduli of
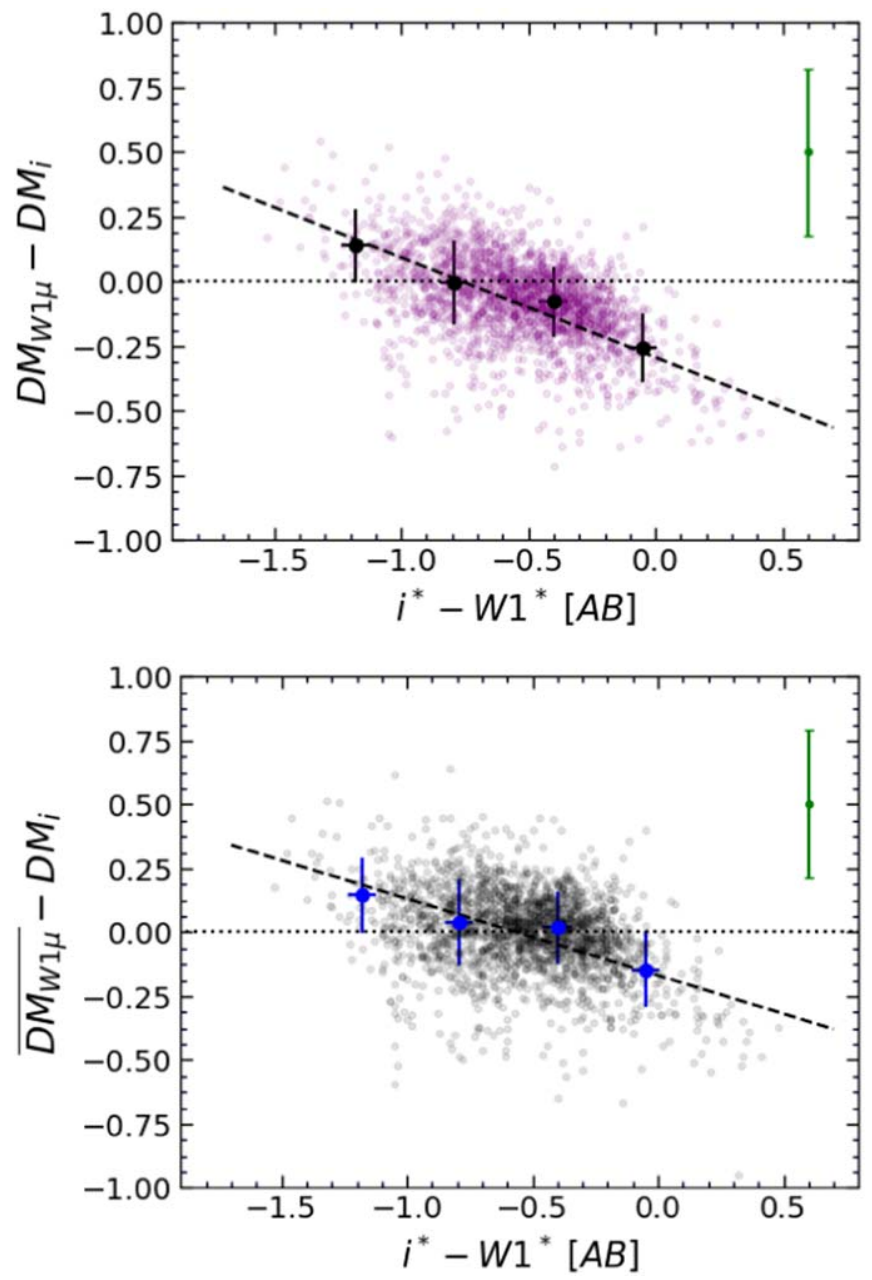

Figure 28. Top: distance moduli offset of the field galaxies vs. $i^{*}-W 1^{*}$ color Other details are the same as the top panel of Figure 27. Bottom: same as the top panel but with the bias-corrected $\overline{\mathrm{DM}_{W 1 \mu}}$.

$\mathrm{DM}_{W 1 \mu}+0.11$ and $\mathrm{DM}_{W 2 \mu}+0.17$. These offsets are listed as $\triangle \mathrm{DM}$ in column (8) of Table 10 and included to form the adjusted moduli, $\mathrm{DM}_{c}$.

These discrepancies arise from the still-modest size of the zero-point calibrator sample that does not fairly represent the global distribution of galaxies. The bias is evaluated with improved statistics using the field galaxies of the impending Cosmicflows-4 distance compilation. We take as a baseline $\mathrm{DM}_{i}$ rather than $\mathrm{DM}_{W 1}$ because, as shown in Figure 24, cluster distance moduli measured at the $i$ and $W 1$ bands are reasonably consistent, and with this choice, we can test for other discrepancies between measured moduli at optical and infrared passbands.

The top panels of Figures 27 and 28 plot the $\mathrm{DM}_{W 1 \mu}-\mathrm{DM}_{i}$ of the field galaxies versus their line widths and $i^{*}-W 1^{*}$ colors, respectively. It would be preferable to use the $i^{*}-W 1^{*}$ color term to redress the observed trends because the ITFR already uses line widths to measure distances. However, the $i^{*}-W 1^{*}$ color does not provide a practical solution if optical photometry is missing. The correlation between color terms and line width shown in Figure 12 provides a route to the bias correction that is available for all of our samples.

The black dashed curve in the top panel of Figure 27 shows our best fit to the offsets of moduli as a quadratic function of line width, shifting the $\mathrm{DM}_{W 1 \mu}$ values to the same level as $\mathrm{DM}_{i}$. 

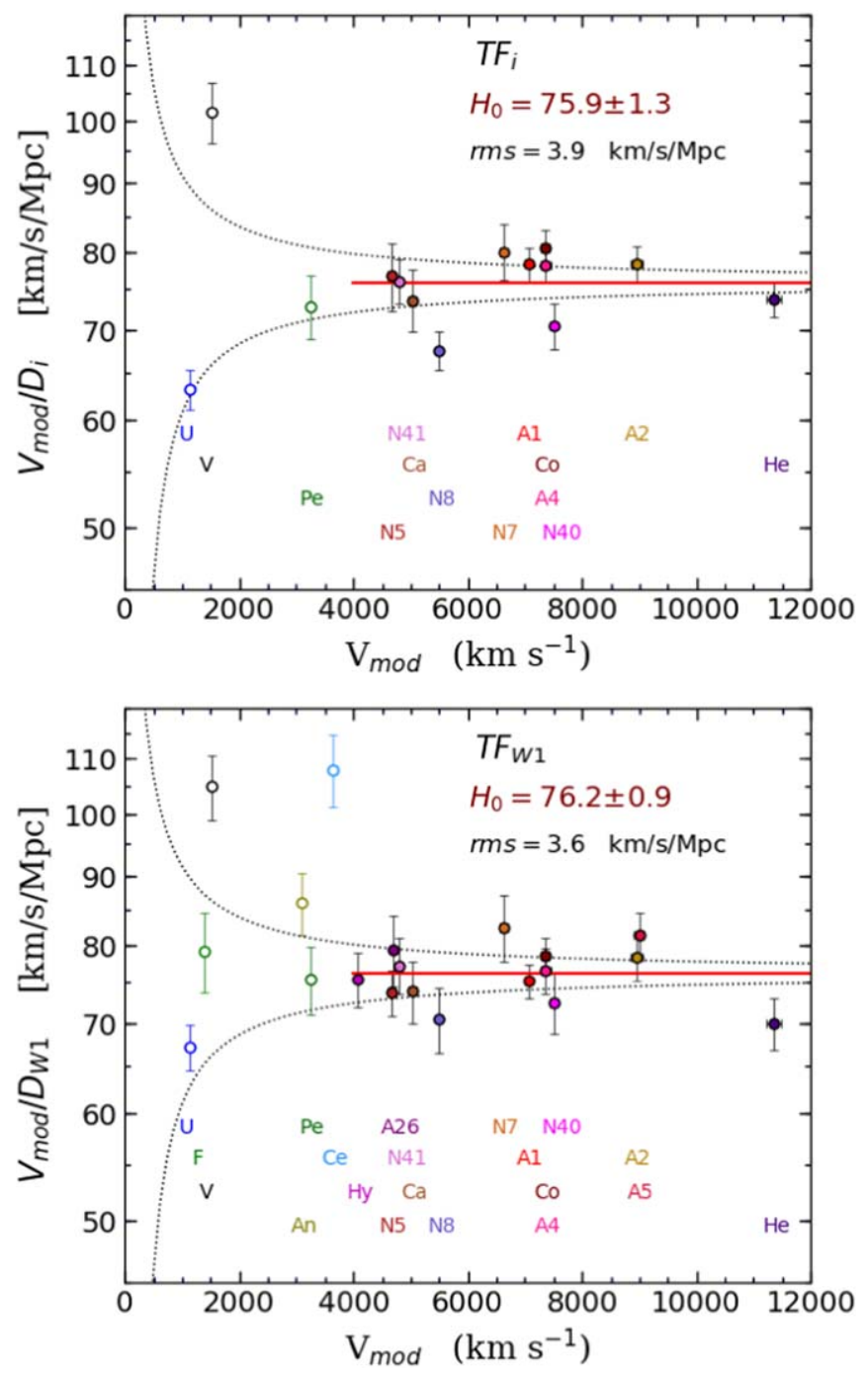

Figure 29. Hubble parameter as a function of systemic velocity at the SDSS $i$ (top) and WISE $W 1$ (bottom) bands. Dotted curves display a $\pm 200 \mathrm{~km} \mathrm{~s}^{-1}$ envelope in velocities. Red horizontal lines lie at the log average of the Hubble parameter of clusters beyond $4000 \mathrm{~km} \mathrm{~s}^{-1}$, represented by filled circles. Corresponding codes are horizontally placed by velocity. Clusters that do not contribute to the averaging process are denoted by open circles.

The offsets of the modified distance moduli, $\overline{\mathrm{DM}_{W 1 \mu}}-\mathrm{DM}_{i}$, are plotted in the bottom panels of Figures 27 and 28 .

In the bottom panel of Figure 27 , the red filled points represent the zero-point calibrators after adjustments following the description in the caption for the top panel of this figure. The gray points illustrate the related adjusted values for the full field sample. On average, the field sample manifests a vertical drift of $0.11 \pm 0.08 \mathrm{mag}$ in the negative direction. The value of this net offset is in agreement with the moduli offset in the top right panel of Figure 26. Evidently, the pattern of surface brightness corrections imposed on the zero-point calibrators does not tightly follow that of the field galaxies. It is expected that this discrepancy would be neutralized by adopting a larger/fairer sample of zero-point calibrators.

Comparing the two panels of Figure 27, we infer that our quadratic formulation based on line width significantly but imperfectly improves the consistency of the distance measurements between $\mathrm{TF}_{W 1 \mu}$ and $\mathrm{TF}_{i}$ alternatives. In this paper, our focus is on galaxy clusters that contain spirals with a variety of properties such that, on average, wavelength discrepancies are expected to be moderate. In applications to the full field sample, discussed in a follow-up paper, concerns for bias in distances to individual galaxies due to wavelength-dependent color variations will require attention.

\section{7. $H_{0}$ from Clusters}

For each cluster, $j$, the Hubble parameter is calculated as $H_{j}=V_{\text {mod, } j} / D_{j}$. The values of the Hubble parameters based on different TFR models are listed in column (11) of Table 10. Figure 29 provides plots of the resulting $H_{j}$ given the distance moduli measured at the $i$ and $W 1$ bands using unadjusted TFR fits. Large scatters are observed for clusters within $4000 \mathrm{~km} \mathrm{~s}^{-1}$ due to substantial peculiar motions within our host supercluster complex. In Figure 29, dotted lines show an error envelope of $\pm 200 \mathrm{~km} \mathrm{~s}^{-1}$ to illustrate how the Hubble parameter is influenced by peculiar velocities as a function of distance. We find a Hubble constant of $H_{0}=75.9 \pm 1.3 \mathrm{~km} \mathrm{~s}^{-1} \mathrm{Mpc}^{-1}$ based on the unadjusted TFR analysis at the $i$ band with an rms scatter of $3.9 \mathrm{~km} \mathrm{~s}^{-1} \mathrm{Mpc}^{-1}$. For the unadjusted TFR at the $W 1$ band, $H_{0}=76.2 \pm 0.9 \mathrm{~km} \mathrm{~s}^{-1} \mathrm{Mpc}^{-1}$ with an rms scatter of $3.6 \mathrm{~km} \mathrm{~s}^{-1} \mathrm{Mpc}^{-1}$.

Figures 30 and 31 present Hubble parameter derivations from selected formulations labeled in the fourth column of Table 9. The cluster moduli determined at optical bands are in good agreement with each other; hence, there is no motivation for adjustments to cluster moduli measured at optical passbands. However, the cluster moduli derived from the adjusted models at infrared bands, $\mathrm{DM}_{W 1 c}, \mathrm{DM}_{W 2 c}, \mathrm{DM}_{W 1 \mu}$, and $\mathrm{DM}_{W 2 \mu}$, must receive offsets to be on the same scale as the measured moduli at optical bands. We apply the offset values shown in Figure 26 to the moduli prior to the calculation of the Hubble parameters.

The Hubble constant values given in Figures 30 and 31 are consistent within the claimed statistical uncertainties. The rms scatter of the Hubble parameters is larger than the statistical uncertainties owing to cluster peculiar velocities. It may well be that our calibration clusters do not extend to distances such that peculiar velocities are negligible. In addition, any systematic errors in the distances of the zero-point calibrators systematically affect our measurements of $H_{0}$. The main purpose of this study is not to determine the absolute value of $H_{0}$ but rather to provide calibrations of the TFR methodology for measuring relative galaxy distances that are consistent across passbands and parameterizations.

\section{Conclusions}

Every bit of information used in the calibration of the luminosity-line width relations of spirals might introduce biases and uncertainties in the final derived relations. The main purpose of this calibration program is to determine the peculiar flow of galaxies; thus, any unaccounted biases might generate artificial flows suggesting unrealistic cosmological structures. In this program, our main focus is to obtain distances at multiple passbands that are in good agreement with each other and on the same scale.

The quality of photometry data, H I line widths, and measurements of inclinations are all important inputs. Corrections for dust extinction can be important toward blueward bands, and our formalism to measure dust attenuation (Kourkchi et al. 2019) has been constructed based on the same catalog of spirals used for the current study. 


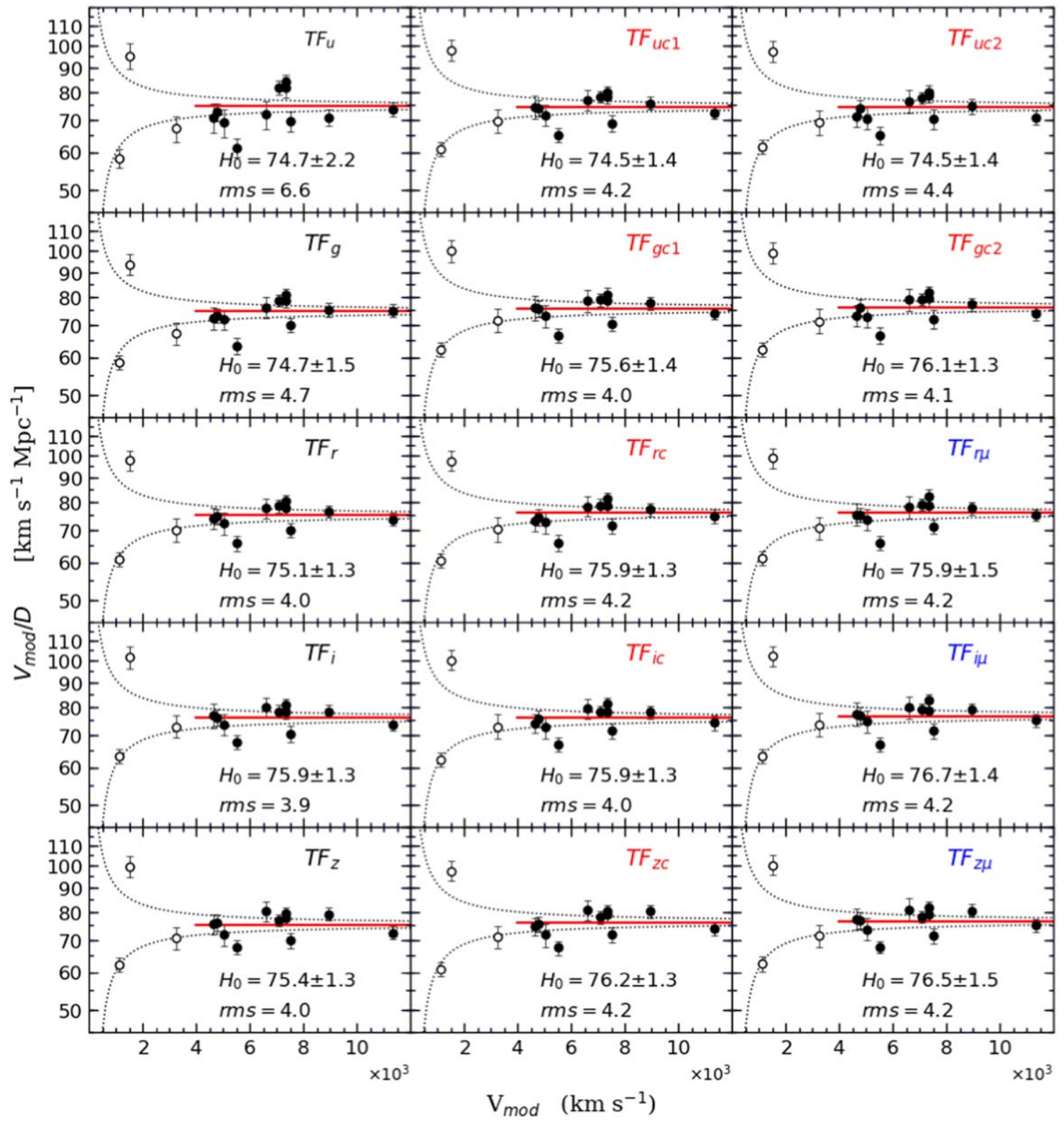

Figure 30. Same as Figure 29 for the optical TFR models presented in Table 9. The code of each model is displayed in the top right corner of each panel.

The TFR calibrating process has two major steps: definition of slopes at each passband and establishment of zero-points. The derivations of slopes of the TFR from an ensemble of cluster galaxies is a robust process, but small variations in the properties of galaxies in each cluster influence that cluster's measured distance relative to the other clusters in the ensemble. For instance, clusters with, on average, redder galaxies are located at farther distances at redward bands.

Zero-point calibrators are the foundation of our calibration process, and any systematic in their distances propagates through the entire process. We separately examined the applicability of two sets of distance calibrators, one using Population I Cepheid variables and the other using Population II red giant branch stars. We find consistency of scales between the two calibrations as manifested in implied values of $H_{0}$ using the scale for Cepheids given by Riess et al. (2019) and the scale for TRGB luminosities given by Rizzi et al. (2007). The TRGB scale advocated by Freedman et al. (2019) gives inconsistency with the Cepheid scale. This alternate scaling does caution us to the plausible existence of zero-point systematics at the level of $3 \%$.

We find good agreement in TFR distances measured at the SDSS $r, i$, and $z$ bands and WISE $W 1$ and $W 2$ bands. The SDSS $u$ and $g$ bands are less useful for determining TFR distances, but the broad span of wavelength coverage they provide offers insight into obscuration and color term issues. Color or surface brightness correlations do not significantly reduced TFR scatter at the optical bands, so they are not used. However, infrared TFR scatter can be substantially reduced with either optical to infrared color or surface brightness terms. The color correlations are strongest but practically not so useful, since the availability of optical information negates the need for an infrared-based measurement. A surface brightness adjustment can be constructed with only infrared photometric information. However, the surface brightness-adjusted TFR at the $W 1$ and $W 2$ bands must be compensated for offsets evidently due to statistical differences between the surface brightness properties of the zero-point calibrators and the full field sample. The offset 


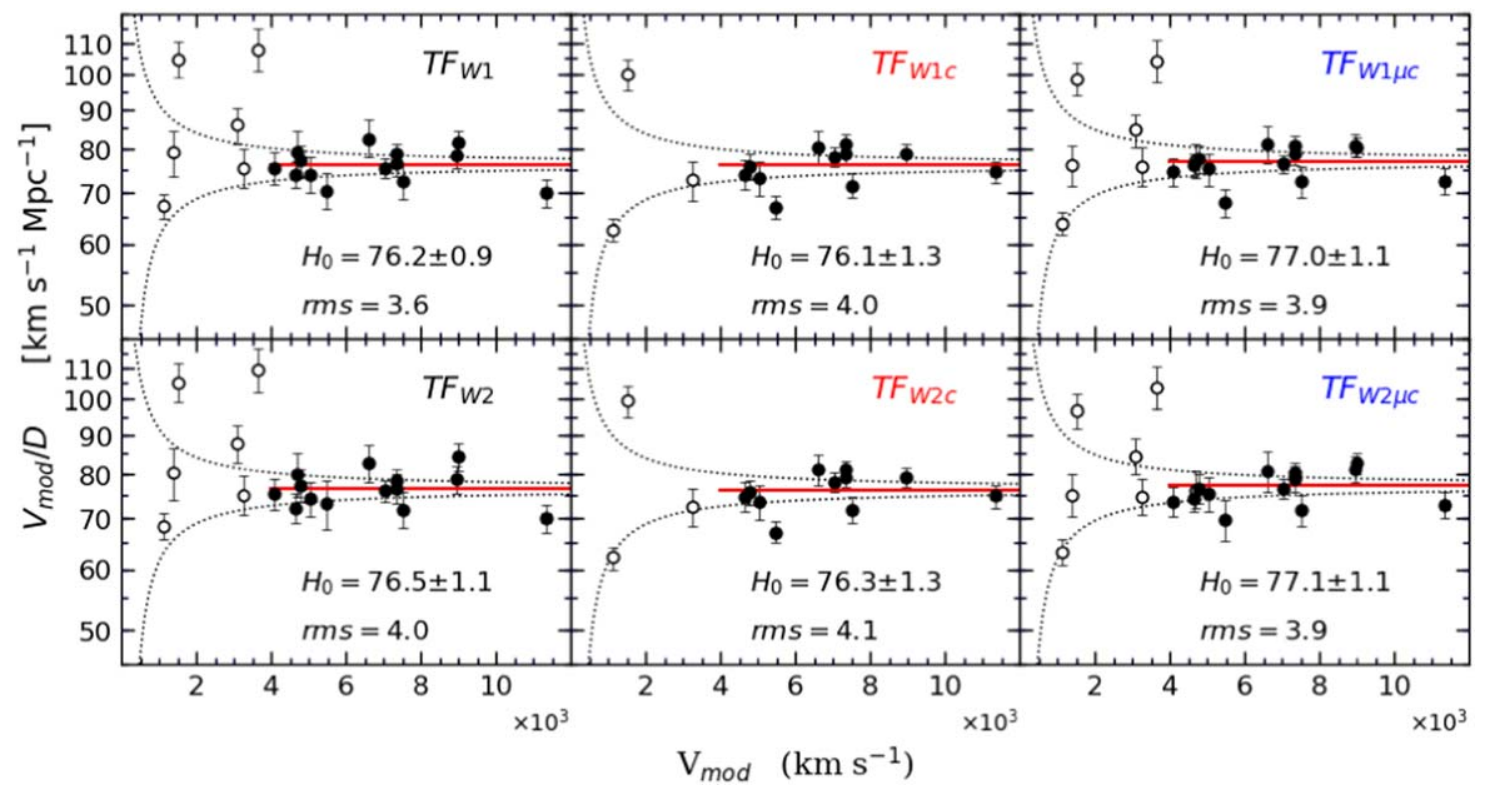

Figure 31. Same as Figure 30 for the infrared ITFR models.

at the level of $5 \%$ is a cautionary example of possible systematics.

The TFR scatter varies with luminosity, such that it is smaller at the bright end and increases toward fainter galaxies. The scatter at the slow-rotating, faint end of the TFR can be reduced by accounting for substantial contributions of dark baryons in the form of neutral gas (McGaugh et al. 2000). We have not explored this added complexity. In the redshift regime $3000-10,000 \mathrm{~km} \mathrm{~s}^{-1}$, where the numbers in our field sample peak, our multiband measurements will typically render distance with $20 \%$ accuracy.

There is a slight curvature at the bright end of the TFR, an effect that has been noticed by others. The departure from linearity in the relation between magnitude and log line width results in a small bias. Another bias results from asymmetric scatter due to magnitude uncertainties in the regime of the Schechter function exponential cutoff. These two biases act in opposite senses, and simulations demonstrate that they roughly but not entirely cancel.

Measurements of distances of clusters at $V_{\text {mod }}>4000$ $\mathrm{km} \mathrm{s}^{-1}$ give a preliminary determination of the Hubble constant, $H_{0}$. The sample is small, 11 optical and 14 infrared, and peculiar velocities may not be negligible. In any event, our measurements of $H_{0}$ at all passbands based on the adjusted and unadjusted TFR models are consistent. We find $H_{0}=$ $75.9 \pm 1.3$ and $76.2 \pm 0.9 \mathrm{~km} \mathrm{~s}^{-1} \mathrm{Mpc}^{-1}$ for the unadjusted TFR at the $i$ and $W 1$ bands. With these values, a 3\% systematic offset in the distance zero-point translates to a $\pm 2.3 \mathrm{~km} \mathrm{~s}^{-1}$ $\mathrm{Mpc}^{-1}$ systematic error in the estimated Hubble constant.

In this study, we only considered biases in the distance measurements of the clusters, where constituent galaxies are treated as an ensemble. There can be additional biases in measuring the distances of individual field galaxies. This issue is important, especially if different passbands are utilized in different parts of the sky. Even if color terms do not lower the scatter of the calibrated relations at the $r, i$, and $z$ bands, they might still help cancel systematics with application to field galaxies. In a subsequent study, we will investigate the possible ways to reduce biases in the distance measurements of field spirals and present formulations for getting consistent distances at optical and infrared bands based on the calibrations provided in this paper.

We are pleased to acknowledge the citizen participation in scientific research of undergraduate students at the University of Hawaii; members of amateur astronomy clubs in France at the Planétarium de Vaulx-en-Velin, Association Clair d'étoiles et Brin d'jardin, Société astronomique de Lyon, Club d'astronomie Lyon Ampère, Club d'astronomie des monts du lyonnais, and Club d'astronomie de Dijon; and friends who helped us with measuring inclinations of spiral galaxies in our sample.

Support for E.K. and R.B.T. was provided by NASA through grant No. 88NSSC18K0424 and for G.A. and R.B.T. through grants from the Space Telescope Science Institute. $\mathrm{H}$. C. acknowledges support from Institut Universitaire de France.

This research has made use of the NASA/IPAC Extragalactic Database, ${ }^{16}$ which is operated by the Jet Propulsion Laboratory, California Institute of Technology, under contract with the National Aeronautics and Space Administration.

\section{ORCID iDs}

Ehsan Kourkchi (10) https://orcid.org/0000-0002-5514-3354

R. Brent Tully (i) https://orcid.org/0000-0002-9291-1981

Gagandeep S. Anand (i) https://orcid.org/0000-00025259-2314

Hélène M. Courtois (iD https://orcid.org/0000-0003-0509-1776

James D. Neill (iD https://orcid.org/0000-0002-0466-1119

Luca Rizzi (iD https://orcid.org/0000-0003-0882-2327

Mark Seibert (iD https://orcid.org/0000-0002-1143-5515

\section{References}

Aaronson, M., Bothun, G., Mould, J., et al. 1986, ApJ, 302, 536 Aaronson, M., Huchra, J., \& Mould, J. 1979, ApJ, 229, 1 Alam, S., Albareti, F. D., Allende Prieto, C., et al. 2015, ApJS, 219, 12

\footnotetext{
16 http://ned.ipac.caltech.edu/
} 
Beers, T. C., Flynn, K., \& Gebhardt, K. 1990, AJ, 100, 32

Bhardwaj, A., Kanbur, S. M., Macri, L. M., et al. 2016, AJ, 151, 88

Courtois, H. M., Tully, R. B., Fisher, J. R., et al. 2009, AJ, 138, 1938

Courtois, H. M., Tully, R. B., Makarov, D. I., et al. 2011, MNRAS, 414, 2005

Djorgovski, S., \& Davis, M. 1987, ApJ, 313, 59

Dressler, A., Lynden-Bell, D., Burstein, D., et al. 1987, ApJ, 313, 42

Du, W., Cheng, C., Zheng, Z., \& Wu, H. 2020, AJ, 159, 138

Fisher, J. R., \& Tully, R. B. 1981, ApJS, 47, 139

Freedman, W. L., Madore, B. F., Gibson, B. K., et al. 2001, ApJ, 553, 47

Freedman, W. L., Madore, B. F., Hatt, D., et al. 2019, ApJ, 882, 34

Gieren, W., Górski, M., Pietrzyński, G., et al. 2013, ApJ, 773, 69

Giovanelli, R., Haynes, M. P., Herter, T., et al. 1997, AJ, 113, 53

Haynes, M. P., Giovanelli, R., Kent, B. R., et al. 2018, ApJ, 861, 49

Haynes, M. P., Giovanelli, R., Martin, A. M., et al. 2011, AJ, 142, 170

Huchra, J. P., Macri, L. M., Masters, K. L., et al. 2012, ApJS, 199, 26

Huchtmeier, W. K., \& Richter, O. G. 1989, A General Catalog of HI Observations of Galaxies. The Reference Catalog (New York: Springer)

Humphreys, E. M. L., Reid, M. J., Moran, J. M., Greenhill, L. J., \& Argon, A. L. 2013, ApJ, 775, 13

Jacobs, B. A., Rizzi, L., Tully, R. B., et al. 2009, AJ, 138, 332

Jang, I. S., \& Lee, M. G. 2015, ApJ, 807, 133

Jang, I. S., \& Lee, M. G. 2017, ApJ, 836, 74

Kourkchi, E., \& Tully, R. B. 2017, ApJ, 843, 16

Kourkchi, E., Tully, R. B., Neill, J. D., et al. 2019, ApJ, 884, 82

Leavitt, H. S., \& Pickering, E. C. 1912, HarCi, 173, 1

Lee, M. G., Freedman, W. L., \& Madore, B. F. 1993, ApJ, 417, 553

Lelli, F., McGaugh, S. S., \& Schombert, J. M. 2016, ApJL, 816, L14

Lelli, F., McGaugh, S. S., Schombert, J. M., Desmond, H., \& Katz, H. 2019, MNRAS, 484, 3267

Magoulas, C., Springob, C. M., Colless, M., et al. 2012, MNRAS, 427, 245

Makarov, D., Prugniel, P., Terekhova, N., Courtois, H., \& Vauglin, I. 2014, A\&A, 570, A13

Makarov, D. I., Zaitseva, N. A., \& Bizyaev, D. V. 2018, MNRAS, 479, 3373

Masters, K. L., Springob, C. M., Haynes, M. P., \& Giovanelli, R. 2006, ApJ, 653,861

McGaugh, S. S. 2005, ApJ, 632, 859

McGaugh, S. S., Schombert, J. M., Bothun, G. D., \& de Blok, W. J. G. 2000, ApJL, 533, L99

Monson, A. J., Freedman, W. L., Madore, B. F., et al. 2012, ApJ, 759, 146

Neill, J. D., Seibert, M., Tully, R. B., et al. 2014, ApJ, 792, 129

Noordermeer, E., \& Verheijen, M. A. W. 2007, MNRAS, 381, 1463

Phillips, M. M. 1993, ApJL, 413, L105
Pietrzyński, G., Graczyk, D., Gallenne, A., et al. 2019, Natur, 567, 200

Planck Collaboration, Aghanim, N., Akrami, Y., et al. 2018, arXiv:1807.06209

Ponomareva, A. A., Verheijen, M. A. W., \& Bosma, A. 2016, MNRAS, 463, 4052

Ponomareva, A. A., Verheijen, M. A. W., Peletier, R. F., \& Bosma, A. 2017, MNRAS, 469, 2387

Querejeta, M., Meidt, S. E., Schinnerer, E., et al. 2015, ApJS, 219, 5

Riess, A. G., Casertano, S., Yuan, W., Macri, L. M., \& Scolnic, D. 2019, ApJ, 876,85

Riess, A. G., Macri, L. M., Hoffmann, S. L., et al. 2016, ApJ, 826, 56

Rizzi, L., Tully, R. B., Makarov, D., et al. 2007, ApJ, 661, 815

Rubin, V. C., Burstein, D., Ford, W. K. J., \& Thonnard, N. 1985, ApJ, 289, 81

Sakai, S., Mould, J. R., Hughes, S. M. G., et al. 2000, ApJ, 529, 698

Schechter, P. 1976, ApJ, 203, 297

Schechter, P. L. 1980, AJ, 85, 801

Scowcroft, V., Freedman, W. L., Madore, B. F., et al. 2011, ApJ, 743, 76

Sorce, J. G., Courtois, H. M., Tully, R. B., et al. 2013, ApJ, 765, 94

Springob, C. M., Haynes, M. P., Giovanelli, R., \& Kent, B. R. 2005, ApJS, 160,149

Springob, C. M., Magoulas, C., Colless, M., et al. 2014, MNRAS, 445, 2677

Springob, C. M., Masters, K. L., Haynes, M. P., Giovanelli, R., \& Marinoni, C. 2007, ApJS, 172, 599

Tonry, J., \& Schneider, D. P. 1988, AJ, 96, 807

Tully, R. B. 1988, Natur, 334, 209

Tully, R. B. 2015, AJ, 149, 171

Tully, R. B., \& Courtois, H. M. 2012, ApJ, 749, 78

Tully, R. B., Courtois, H. M., Dolphin, A. E., et al. 2013, AJ, 146, 86

Tully, R. B., Courtois, H. M., \& Sorce, J. G. 2016, AJ, 152, 50

Tully, R. B., \& Fisher, J. R. 1977, A\&A, 54, 661

Tully, R. B., Mould, J. R., \& Aaronson, M. 1982, ApJ, 257, 527

Tully, R. B., \& Pierce, M. J. 2000, ApJ, 533, 744

Tully, R. B., Shaya, E. J., Karachentsev, I. D., et al. 2008, ApJ, 676, 184

Tully, R. B., Verheijen, M. A. W., Pierce, M. J., Huang, J.-S., \& Wainscoat, R. J. 1996, AJ, 112, 2471

Ulaczyk, K., Szymański, M. K., Udalski, A., et al. 2012, AcA, 62, 247

Verheijen, M. A. W. 2001, ApJ, 563, 694

Willick, J. A. 1994, ApJS, 92, 1

Willick, J. A., Courteau, S., Faber, S. M., et al. 1996, ApJ, 457, 460

Wright, E. L., Eisenhardt, P. R. M., Mainzer, A. K., et al. 2010, AJ, 140, 1868

Yuan, W., Riess, A. G., Macri, L. M., Casertano, S., \& Scolnic, D. M. 2019, ApJ, 886, 61

Zgirski, B., Gieren, W., Pietrzyński, G., et al. 2017, ApJ, 847, 88 